\title{
DETERMINING TEMPERATURE LIMITS OF DRILLING FLUIDS
}

by

\author{
John B. Thuren \\ Martin E. Chenevert \\ Woon-Tsing Wally Huang \\ Edwin Szymanski \\ Philip Arkeketa.
}

UNIVERSITY OF OKLAHOMA

Norman, Oḳlahoma 73069

MASTER

January 1979

Work Performed Under the United States Energy Research and Development Administration Contract No: $E(40-1)-5218$. 


\section{DISCLAIMER}

This report was prepared as an account of work sponsored by an agency of the United States Government. Neither the United States Government nor any agency Thereof, nor any of their employees, makes any warranty, express or implied, or assumes any legal liability or responsibility for the accuracy, completeness, or usefulness of any information, apparatus, product, or process disclosed, or represents that its use would not infringe privately owned rights. Reference herein to any specific commercial product, process, or service by trade name, trademark, manufacturer, or otherwise does not necessarily constitute or imply its endorsement, recommendation, or favoring by the United States Government or any agency thereof. The views and opinions of authors expressed herein do not necessarily state or reflect those of the United States Government or any agency thereof. 


\section{DISCLAIMER}

Portions of this document may be illegible in electronic image products. Images are produced from the best available original document. 
FINAL REPORT

ON

CONTRACT NO. $\mathrm{E}(40-1) 5218$

SUBMITTED TO

SANDIA IABORATORIES

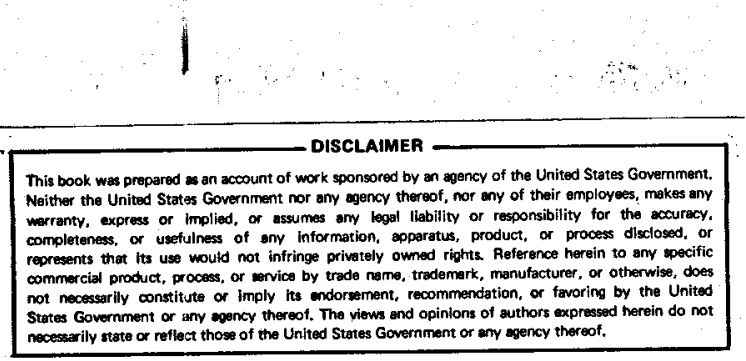

MASTER 
ABSTRACT

A capillary three tube viscometer has been designed which allows the measurement of rheological properties of time dependent non-Newtonian fluids in laminar flow at high temperature and pressure. The objective of this investigation is to detemine the temperature stability of claywater suspensions containing various drilling fluid additives: The additives studied consisted of viscosifiers, filtrate reducers, and chemical thinners. The temperature range studied is from room temperature to 550 degrees Fahrenheit: The system pressure is consistently maintained above the vapor pressure.

The Bentonite and water standardized base mud used is equivalent to a 25 PPB fluid. Stabilization of the base mud is necessary to obtain steady state laminar flow conditions and to obtain reliable temperature thinning effects with each temperature interval under investigation. Generally the temperature levels are maintained for one hour until $550^{\circ} \mathrm{F}$ is attained. The last interval is then maintained until system fluid degradation occurs.

Rheological measurements are obtalned from differential pressure transducers located in a three diameter tube test section and externally at ambient conditions from a Baroid Rotational Viscometer. The power law model for non-Newtonlan fluids is used to correlate the data. 
Abstract ....................... . . ii

List of Tables . . . . . . . . . . . . . . . . . vi

List of Illustrations. . . . . . . . . . . . . . vili

Introduction ....................... 1

Three Tube Viscometer Tests. . . . . . . . . . . . . . 3

Fluid Classification . . . . . . . . . . . . 3

Experimental Equipment... . . . . . . . . . . 6

Fluid Preparation. ................ 10

Test Procedure . . . . . . . . . . . . . . . . . . . 13

Filtrate Reducers, Viscosifiers, and Chemical Thinners . 15

Lignosulfonates and Lignites . . . . . . . . . . . 17

Test Results ..................... 18

Phase I. . . . . . . . . . ..... 19

Phase II................. 19

Phase III. . . . . . . . . . . . . 20

Polyanionic Cellulose. . . . . . . . . . 21

HEC. . . . . . . . . . . . . . . . 21

Guar Gum . . . . . . . . . . . . . . 22

Modifled Polysaccarlde .............. 23

Ferrochrome Lignosulfonate . . . . . . . . . 24

Chrome Lignosulfonate. . . . . . . . . . . 25

Chrome Lignosulfonate With Modif led Lignite A. . . . 26

Chrome Lignosulfonate With Modified Lignite B. . . . 26

Appendix A . . . . . . . . . . . . . . . 90

I. Introduction ................ 91

II. Related Problems ................. . . 94

III. Preliminary Design Considerations. . . . . . . . . 96

IV. Behavior of Drilling Fluids at Elevated Temperatures . . . 98

v. Selection of Heat Transfer Units . . . . . . . . 102

Steam. . . . . . . . . . . . . . . 102

External Electric Heating. . . . . . . . . 102

Internal Electric Heating. . . . . . . . . . 104

Heat Transfer Liquids. . . . . . . . . . 105 
VI. Evaluation of Thermal Parameters of Drilling Fluids. . . 109

VII. Sizing of the Mud-To-Mud Heat Exchanger. . . . . . . 113

VIII.. Sizing of the Electrical Heating Section . . . . . . . 121 Associate Fouling Problems............ . 122

IX. Sizing of the Molten Salt Heat Exchanger . . . . . . . 125 Safety Measures. . . . . . . . . . . 129

X. Sizing of the Water Cooler ............. . 132

XI. 3 Tube Viscometer. . . . . . . . . . . . . 134

XII. The System . . . . . . . . . . . . . . . 138

Measuring and Recording Instruments. . . . . . . 140

Other Test Apparatus............. . 142

XIII. Test Controls. .................. 143

Preliminary Tests. . . . . . . . . . . 143

Experimental Testing............. 144

Nomenclature . . . . . . . . . . . . . . . . 149

Appendix B . . . . . . . . . . . . . . . 152

Introduction . . . . . . . . . . . . . . . 153

Technical Approach............... 153

Heat Exchanger Shell . . . . . . . . . 153

Drilling Fluid Tubes . . . . . . . . . 155

Drain Systens. . . . . . . . . . . 155

Heating Elements ............... 156

Temperature Controllers. . . . . . . . 157

Properties of Hitec. . . . . . . . . . . 157

Insulation . . . . . . . . . . . . 158

Performance of the Hitec System. . . . . . . 159

Appendix C . . . . . . . . . . . . . . . . 160

Electrical Circuitry for Three Tube Viscometer . . . . . 161

Electrical Circultry ............... . . 161

Electronic Filtering of Pressure Measurements of the

Flow Loop. . . . . . . . . . . . . . 162

Appendix D . . . . . . . . . . . . . . . . . 171

Filtrate Tests.................. 172

Test Procedure .............. 172 
Results. . . . . . . . . . . . . 173

Conclusions. . . . . . . . . . . . . . . 173

Corrosion Tests. . . . . . . . . . . . . 177

Appendix E . . . . . . . . . . . . . . . . . 181

References .................... 182

Appendix F . . . . . . . . . . . . . . . . 185

Bibliography .................. 186 


\section{LIST OF TABLES}

Table

Page

1. Test Section Dimensions ............... 8

2. Barold Rotational Viscometer Data for Bentonite . . . . . 28

3. Pipe Viscometer Data for Bentonite. . . . . . . . . 29

4. Baroid Rotational Viscometer Data for CMC . . . . . . . 33

5. Pipe Viscometer Data for CMC. . . . . . . . . . . 34

6. Barpld Rotational Vis. Data for Polyanionic Cellulose.... 39

7. Pipe Viscometer Data for Polyanionic Cellulose. ....... 40

8. Baroid Rotational Viscometer Data for Guar Gum. . . . . . . 44

9. Pipe Viscometer Data for Guar Gum .............. 45

10. Barold Rotational Viscometer Data for Polysaccaride . . . . 49

11. Pipe Viscometer Data for Polysaccaride. . . . . . . . . 50

12. Baroid Rotational Viscometer Data for Ferrochrome IIgnosulfonate. . . . . . . . . . . . . 53

13. Pipe Viscometer Data for Ferrochrome Lignosulfonate ... . . 54

14. Baroid Rotational Viscometer Data for Chrome Lignosulfonate - 56

15. Pipe Viscometer Data for Chrome Lignosulfonate. . . . . . . 57

16. Baroid Rotational Viscometer Data for Chrome Lignosulfonate Lignite A . . . . . . . . . . . . . . . 60

17. Pipe Viscometer Data for Chrome Iignosulfonate - Lignite A. . 61

18. Barold Rotational Viscometer Data for Chorme Lignosulfonate Lignite B . . . . . . . . . . . . . . 65

19. Pipe Viscometer Data for Chrome Lignosulfonate - Lignite B. - 66

20. Thermal Parameters of Major Constituents in Drilling Fluids . 110

21. Comparison of Calculated Thermal Parameters of Drilling

Fluids Based on Mud Weight. . . . . . . . . . . 110 
22. Comparison of Heat Transfer Units Employed . . . . . . . 112

23. Significance of Natural Convection Based Upon the GR/RE ${ }^{2}$ Rat1o........................ 117

24. Sample Calculation of Heating Length For the Mud-To-Mud Heat Exchanger...................... 118

25. Some Parameters of Molten Salt at $600^{\circ} \mathrm{F} . . . . . . . . . .128$

26. Calculated Film Coefficient on the Shell Side of the Molten Salt Heat Exchanger for Different Dimensions and Horsepower Ratings....................... 129

27. Filtrate Tests Results of Chrome Lignosulfonate. . . . . 176

28. Corrosion Tests. . . . . . . . . . . . . . 178 


\section{LIST OF ILLUSTRATIONS}

Figure

Page

1. High Temperature Testing and Stabilization Systems. . . . . 7

2. Shear valve..................... 9

3. Correlation of Rheometer and Flow Loop Viscosity for Bentonite.................... 30

4. Power Law Representation Shear Stress vs Shear Rate for Bentonite at $200^{\circ} \mathrm{F}$..................... 31

5. Power Law Representation Shear Stress vs Shear Rate for

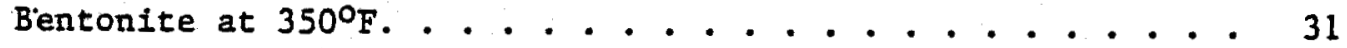

6. Power Law Representation Shear Stress vs Shear Rate for

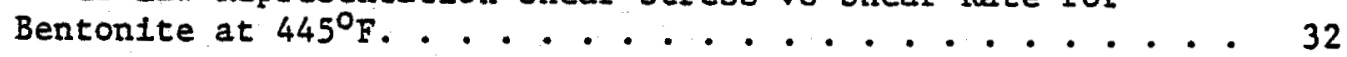

7. Correlation of Rheometer and Flow Loop Viscosity for CMC. - 35

8. Power Law Representation Shear Stress vs Shear Rate for CMC

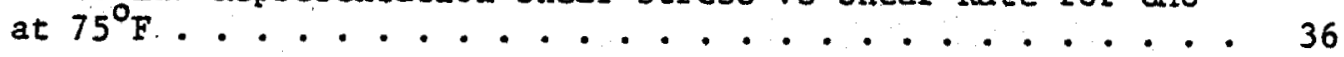

9. Power Law Representation Shear Stress vs Shear Rate for MC

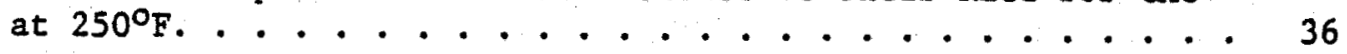

10. Power Law Representation Shear Stress vs Shear Rate for CMC at $350^{\circ} \mathrm{F} . \ldots \ldots \ldots$

11. Power Law Representation Shear Stress us Shear Rate for CMC at $450^{\circ} \mathrm{F}$. . . . . . . . . . . . . . .

12. Power Law Representation Shear Stress vs Shear Rate for CMC at $550^{\circ} \mathrm{F}$.

13. Correlation of Rheometer and Flow Loop Viscometer for Polyanionic Cellulose . . . . . . . . . . . . .

14. Power Law Representation Shear Stress vs Shear Rate for Polyanionic Cellulose at $75^{\circ} \mathrm{F} . . . . . . . . . .$.

15. Power Law Representation Shear Stress vs Shear Rate for

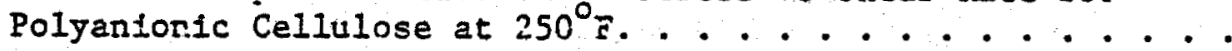

16. Power Law Representation Shear Stress vs Shear Rate for Polyanionic Cellulose at $450^{\circ} \mathrm{F}$.............. 
17. Power Law Representation Shear Stress vs Shear Rate for Polyanionic Cellulose at $550^{\circ} \mathrm{F}$. . . . . . . . . . 43

18. Correlation of Rheometer and Flow Loop Viscometer for Guar

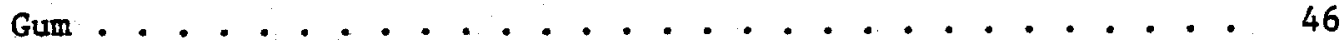

19. Power Law Representation for Shear Stress vs Shear Rate for

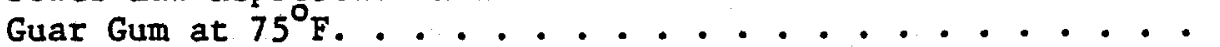

20. Power Law Representation for Shear Stress vs Shear Rate for Guar Gum at $250^{\circ} \mathrm{F}$. . . . . . . . . . . . . . . 47

21. Power Law Representation for Shear Stress vs Shear Rate for Guar Gum at $350^{\circ} \mathrm{F}$. . . . . . . . . . . . . . .

22. Power Law Representation for Shear Stress vs Shear Rate for

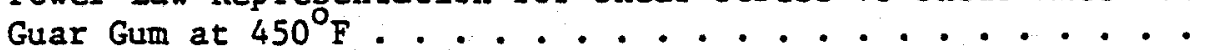

23. Correlation of Rheometer and Flow Loop Viscometer for Polysaccaride . . . . . . . . . . . . . . . 5

24. Power Law Representation Shear Stress vs Shear Rate for

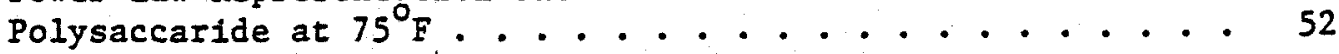

25. Power Law Representation Shear Stress vs Shear Rate for Polysaccaride at $250^{\circ} \mathrm{F}$. . . . . . . . . . . . .

26. Correlation of Rheometer and Flow Loop Viscosity for FerroChrome Lignosulfonate... . . . . . . . . . . 55

27. Correlation of Rheometer and Flow Loop Viscosity for Chrome Lignosulfonate. . . . . . . . . . . . . . . 58

28. Power Law Representation Shear Stress vs Shear Rate for Chrome Lignosulfonate at $450^{\circ} \mathrm{F}$. . . . . . . . . . .

29. Correlation of Rheometer and Flow Loop Viscosity for Chrome Lignosulfonate - Lignite A. . . . . . . . . . . .

30. Power Law Representation Shear Stress vg Shear Rate for Chrome Lignosulfonate - Iignite A at $75^{\circ} \mathrm{F}$. . . . . . 63

31. Power Law Representation Shear Stress vs Shear Rate for Chrome Lignosulfonate - Lignite A at $250^{\circ} \mathrm{F} . \ldots . . . . .$.

32. Power Law Representation Shear Stress $\nabla 5$ Shear Rate for Chrome Lignosulfonate - Lignite A at $350^{\circ} \mathrm{F}$. . . . . . . . 
Figure

Page

33. Power Law Representation Shear Stress vs Shear Rate for Chrome Lignosulfonate - Lignite A at $450^{\circ} \mathrm{F}$. . . . . . . 64

34. Correlation of Rheometer and Flow Loop Viscosity for Chrome Iignosulfonate - Lignite B. . . . . . . . . . . . 67

35. Power Law Representation Shear Stress vs Shear Rate for Chrome Iignosulfonate - Lignite B at $250^{\circ} \mathrm{F}$.........

36. Power Law Representation Shear Stress vs Shear Rate for Chrome Lignosulfonate - Lignite $\mathrm{B}$ at $350^{\circ} \mathrm{F}$. . . . . . . 68

37. Modifled Magcobar Flow Loop................ 71

38. Some Examples of Temperature Dependence of Drilling Fluids in Terms of Flow Behavior Index. . . . . . . . . . . . .

39. Possible Arrangement of Heater \& Tubing in Cross Section to Enhance Heat Transfer by Natural Convection . . . . . . 103

40. Simplified Model of the Brazed Parallel-Tubes ... . . . . 120

41. Flow Pattern of Natural Convection in an Enclosure Heated From the Outside. . . . . . . . . . . . 126

42. Schematic Diagram of the Rotating Cylinder Heat Exchanger . . 126

43. Diagram of Flow Loop. . . . . . . . . . . . 139

44. Filtration Test Unit. ............... 146

45. Shear Valve . . . . . . . . . . . . . . . . 147

46. Corrosion Coupon Holder . . . . . . . . . . . . 148

47. Hitec Heat Exchanger Detail . . . . . . . . . . 154

48. Power Control and Distribution Block Diagram. . . . . . . 164

49. Pressure Recording System . . . . . . . . . . 165

50. Temperature Recording System. . . . . . . . . 166

51. Main Power Switching. . . . . . . . . . . . . 167

52. Test Section Heater Power Distribution and Contro1. . . . . 168

53. 1/3 Hz Filter. . . . . . . . . . . . 169 
Figure

Page

54. Five Channel Gain Control .............. . 170

55. Filtration Test Unit. . . . . . . . . . . . . 174

56. Filtrate Cell Connections . . . . . . . . . . 175

57. Experimental Drilling Fluids Tests. . . . . . . . 179

$x 1$ 


\section{INTRODUCTION}

The current trend of geothermal well drilling has prompted the Department of Energy to embark on a research program to determine the availability of fluids which can tolerate the high temperatures and pressures encountered. Temperature degradation of fluids is considered of primary concern because of the costs involved in maintaining wellbore hydraulics, hole stability and reducing reservoir damage.

Previous investigations into the thermal stability of drilling fluids have been concerned with clay properties such as viscosity, gel strength and flocculation. Rogers ${ }^{35}$, Annis ${ }^{2}$, and Hiller ${ }^{17}$, utilized modified rotational viscometers to analyze clay water suspensions in the 250 to 350 degree Fahrenheit range. These studies indicated that the stability of the fluid is dependent on the temperature, time of exposure and the degree of hydration of the clay platelets. Annis further emphasized that various electrolytes and certain molecules directly influence the fluid behavior at high temperature.

Recent studles have developed flow networks which apply high and Iow shear rates to further analyze fluid behavior. Capillary tube viscometers were used by Pritchard ${ }^{6}$ and Krueger $^{27}$ to evaluate certain clay water suspensions. Pritchard studied lignosulfonate chemical behavior utillzing the aging cell method to expose the chemical to temperature. The aged lignosulfonate was then added to the base fluid 
and evaluated in the capillary tube viscometer. Krueger used a pipe viscometer to shear age his fluids and pressure drop data was obtained for correlating the shear history of the test fluid. These apparatus rely on wall shear and give values of viscosity with known shear rates. These designs were primarily concerned with simulating down hole conditions.

The present configuration has the capability of measuring pressure drop from fluid flow under extreme conditions. The temperature range attainable is limited to $550^{\circ} \mathrm{F}$. The pressure may be maintained from atmospheric to $3,000 \mathrm{ps}$. The flow loop contains shear valves which sirulate bit jet shear rates of, $10,000 \mathrm{sec}^{-1}$ to $50,000 \mathrm{sec}^{-1}$, and are adjustable. The test section shear rates range from $20 \mathrm{sec}^{-1}$. to $1,400 \mathrm{sec}^{-1}$. The differential pressure measurements are accurate to \pm .1 psi, with 50 psi maximum.

The stabilization of the test fluid is of primary importance In attaining steady state conditions for testing. Clay hydration effects the fluid consistency and is time dependent. Therefore a proper hydration period is necessary to stabilize all clay suspensions. Chemical reaction rates also are time dependent. These conditions must be maintained constant or minimized to such a point as to eliminate their effects. The steady flow conditions allows classification of the test fluid, evaluation of shear history, and an understanding of temperature thinning or thickening tendencies. Some non-Newtonfan fluid classification methods will be discussed first. The equipment and test procedure is then presented. Fluid preparation and various stabilization techniques are included. Finally the laboratory results and evaluation of the flow tests are presented. 


\section{THREE TUBE VISCOMETER TESTS}

\section{FLUID CLASSIFICATION}

Current classification schemes divide fluids into two major categories, Newtonian and non-Newtonian. This distinction is based on response characteristics noted under a range of shear stresses. The shear stress will equal some function of the shear rate. Aziz and Grovier ${ }^{15}$ indicate as many as eleven different parameters may effect fluid characteristics. They also indicate that each parameter may also depend on other parameters. Most of the parameters involve macromolecules and particles which compose the fluid. They further state that fluids are classified into varfous rheological types on the basis of graphical analysis.

Newtonian fluids are identified on Cartesian coordinates with an arithmetic plot of shear stress vs shear rate. The data will be linear through the origin for a given temperature and pressure. The slope of the rheogram will be the viscosity. The viscosity will be constant for an increase or decrease in shear rate.

Non-Newtonian fluids do not exhibit a constant proportionality between shear stress and shear rate at constant temperature and pressure. The ratio of shear stress to shear rate is termed apparent viscosity. 
The power law model fits many non-Newtonian fluids at intermediate shear rate ranges but fails at both low and very high shear rate ranges. The fluid will usually exhibit Newtonian characteristics, at the high and low shear rates.

The power law behavior index, $n$, indicates variance from Newtonian behavior. Therefore the farther from unity the more nonNewtonian the fluid has become.

The power law constant, $K$, is a consistency Index. Both parameters are related to the fluid macromolecules and other solid-Ilquid interactions. On a logrithmic plot the intercept at unity shear rate is equated to the consistency index. The greater the consistency index value, $K$, the more viscous the fluid.

If the apparent viscosity should decrease with increasing shear rate the fluid is termed pseudoplastic. This is provided the fluid is In a stable condition and the proper shear ranges are obtained.

The power law model is widely used in industry to analyze drilling fluids. Mathematically it can be written as:

$$
\tau_{y x}=R\left(\frac{d u}{d y}\right)^{n}
$$

where $\tau_{y x}$ is shear stress and $\frac{d u}{d y}$ is shear rate.

Dilatant fluids exhibit an increase in apparent viscosity with increased shear rates. These type fluids may exist only in certain concentration ranges. The degree of dilatancy is directly related to fluid interaction with the particles. The limiting concentration range depends on the particle concentration ranges and physical characteristics 
of the fluid. Generally fluids become pseudoplastic when concentration ranges are lower or higher than the limiting concentration.

The majority of clay suspensions exhibit a yield stress. There are basically three fluid types in this category, Bingham plastic, yield pseudoplastic and yield dilatant. These fluids require a finite shear stress in excess of the yield stress to initiate flow. The rheological. equation takes the form:

$$
\tau_{y x}-\tau_{\text {yield }}=\left\{n^{-}\left(\frac{d u}{d y}\right)\right\}^{\frac{1}{\pi}}
$$

This is often referred to as the Herschel-Bulkley Model.

Bingham fluids exhibit a linear relationship between shear stress and shear rate after overcoming the yield stress. Parameters for Bingham fluids may be obtained from the slope and Intercept of a straight line relationship between shear stress and shear rate. The yield point is equated to the intercept at zero shear rate and the plastic viscosity equals the slope of the straight line. Usually the model provides a reasonable fit in the laminar flow range, expecially at low shear rates. Clay water suspensions fit within a range of fluid concentrations. Yield pseudoplastics vary from pseudoplastics in yield stress requirements only. The stress curve exhibits a convex appearance. Many clay water suspensions behave as yield pseudoplastics especially at Intermediate levels of concentration.

Yield dilatant fluids are similar to dilatant fluids except for the yield stress. The concave character of the shear curve is identical in nature to the dilatant fluids. 
Time dependent fluids are defined as fluids which undergo a thickening or thinning with time when held at a constant shear stress.

This investigation has considered all fluids as power law. The test procedure allows a period of time to shear age the fluid. Many time dependent characteristics are eliminated. Yield stress analysis is neglected other than in rheometer data.

EXPERIMENTAL EQUIPMENT

A diagram of the flow loop used in this study is illustrated in Figure 1. The flow loop has the capability of shearing the test fluids in a similar range to that recorded in an actual wellbore.

The test section is designed to eliminate entrance effects and reduce turbulence formation at the pressure taps. The current configuration of the test section measures data in the $20 \mathrm{sec}^{-1}$ to $1,400 \mathrm{sec}^{-1}$ range. The test section consists of 3 stainless steel tubes. Each tube is insulated to maintain the test temperature. Stainless steel tees are placed in the lines to facilitate installing pressure taps. The pressure taps consist of stainless steel fittings and a capillary line leading to each pressure transducer. The capillary lines are used to dissipate heat to ambient even at the $550^{\circ} \mathrm{F}$ test temperature. Test section dimensions are recorded in Table 1.

System pressure 1s maintained at 3,000 psi with spring loaded shear valves supplied by Dresser Magcobar. The shear valves produce sufficient backpressure to prevent vaporization in the test section. The valves shear the fluids in the $10,000 \mathrm{sec}^{-1}$ to $50,000 \mathrm{sec}^{-1}$ range. A diagram of the shear valve is included in figure 2. 
3 Way Valve Test Section 1 Test Section 2 Test Section 3

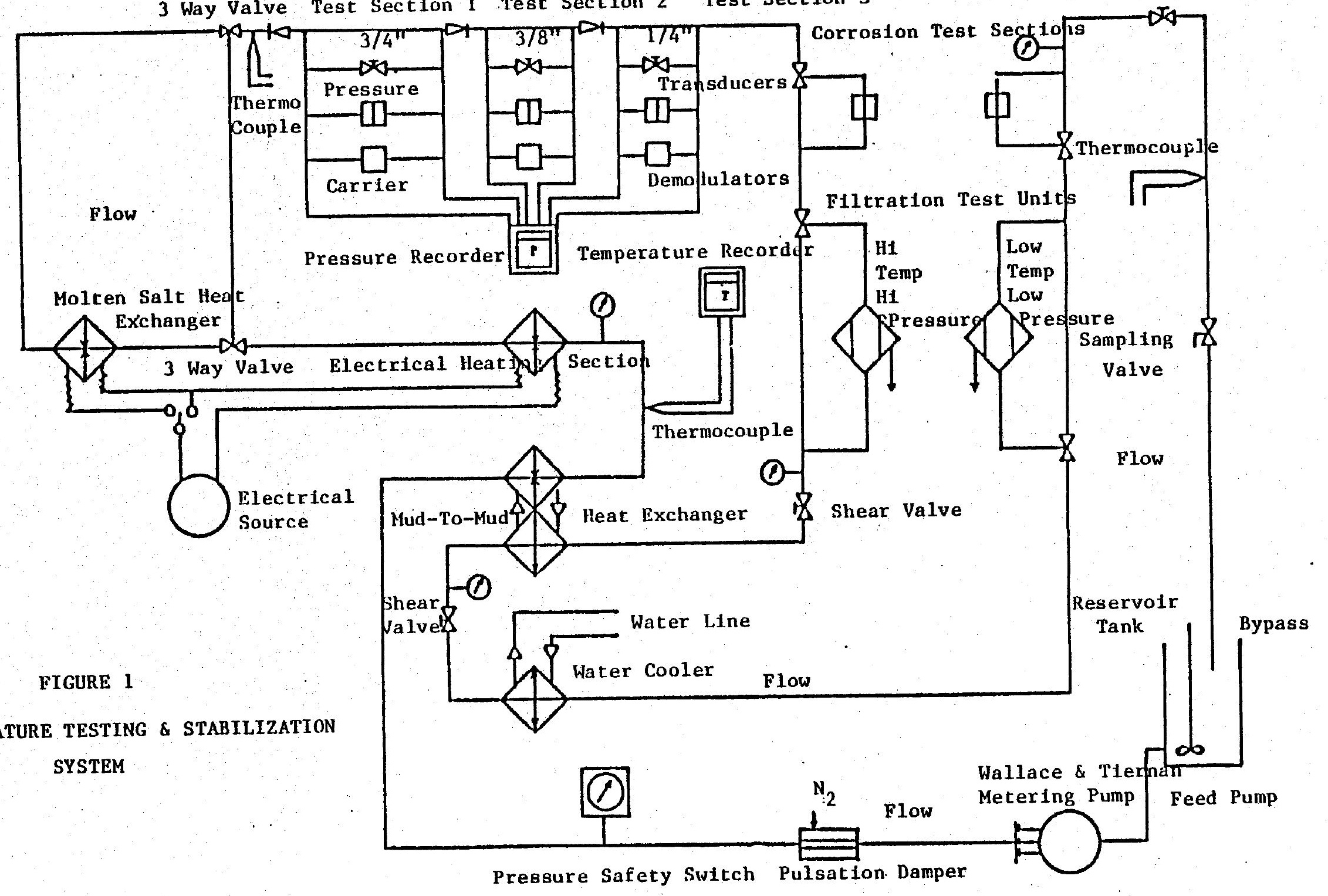


TABLE I

TES: SECTION DIMENSIONS

\begin{tabular}{|c|c|c|c|c|c|c|}
\hline & $\begin{array}{l}\text { Diameter } \\
\text { Ratio }\end{array}$ & $\begin{array}{l}\text { Resistance } \\
\text { Coefficient }\end{array}$ & $\begin{array}{l}\text { Equivalent } \\
\text { Entrance } \\
\text { Length }\end{array}$ & $\begin{array}{l}\text { Calculated } \\
\text { Calming } \\
\text { Length }\end{array}$ & $\begin{array}{l}\text { Actual } \\
\text { Calming } \\
\text { Length }\end{array}$ & $\begin{array}{l}\text { Actual } \\
\text { Tap } \\
\text { Distance }\end{array}$ \\
\hline $\begin{array}{l}\text { Sudden enlarge- } \\
\text { ment between }{ }^{\prime \prime} \\
x .035^{\prime \prime} \text { and } 3 / 4^{\prime \prime} \\
x .065^{\prime \prime} \text { tube }\end{array}$ & 0.29 & 0.84 & 1.68 & $9.1 "$ & $24^{\prime \prime}$ & $4.33^{\prime}$ \\
\hline $\begin{array}{l}\text { Sudden contrac- } \\
\text { tion between } 3 / 4^{\prime \prime} \\
x .065^{\prime \prime} \text { and } 3 / 8^{\prime \prime} \\
x .049^{\prime \prime} \text { tube }\end{array}$ & 0.45 & 0.35 & 0.70 & $5.8 "$ & $24^{\prime \prime}$ & $3.25^{\circ}$ \\
\hline $\begin{array}{l}\text { Sudden contrac- } \\
\text { tion between } 3 / 8^{\prime \prime} \\
x .049^{\prime \prime} \text { and } 1 / 4^{\prime \prime} \\
x .035^{\prime \prime} \text { tube }\end{array}$ & 0.65 & 0.25 & 0.50 & $2.7^{\prime \prime}$ & $24 "$ & $3.25^{\prime}$ \\
\hline $\begin{array}{l}\text { Sharp edged } \\
\text { entrance }\end{array}$ & 0 & 0.50 & 1.0 & $\begin{array}{c}30 x \\
\text { diameter }\end{array}$ & $-\cdots$ & -.-- \\
\hline
\end{tabular}


FIGURE 2

SHEAR VALVE

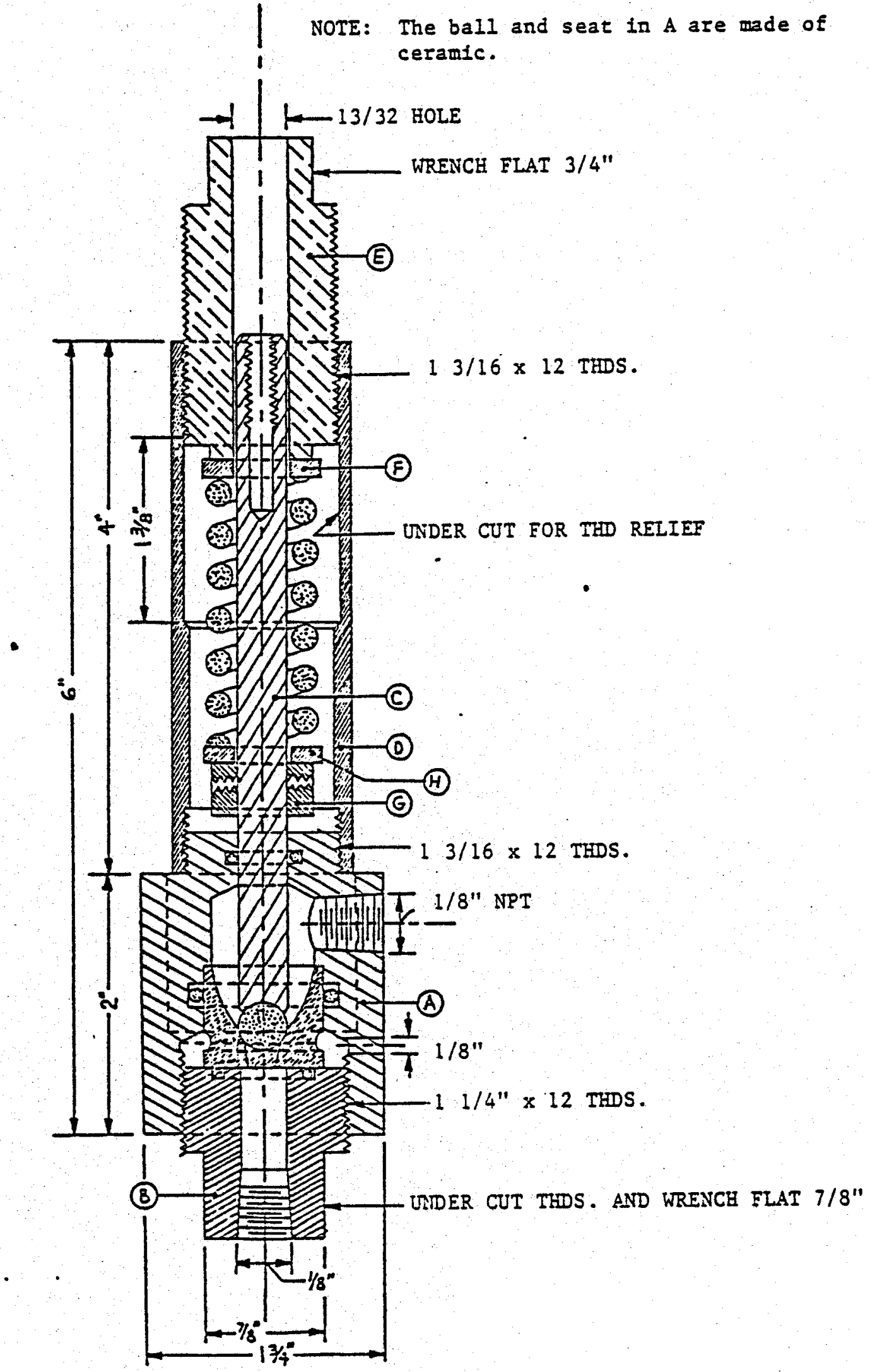


The flow loop temperature is obtained by the use of electrical tubular heaters attached to the $\frac{1}{4}$ inch flow tubing. The tubulars are rated at $2 \mathrm{KW}$ each and are 90 inches 10ng. There are 9 tubular heaters in the flow loop. Three temperature controllers monitor and maintain fluid temperature in the loop. The system is capable of attaining $550^{\circ} \mathrm{F}$. Flow is maintained with a Wallace and Tiernan metering pump. The pump is a positive displacement pump without suction. Therefore a feed pump and variable speed electrical drive motor were required to maintain flow from the reservoir to the metering pump liquid ends. The feed pump included a bypass $100 p$ and valve to return fluid to the reservoir, this allowed pressure control to the metering pump. The bypass network also allows extra mud mixing capabilities.

\section{FIUID PREPARATION}

Two standard base muds were prepared. The base fluids consisted of a $25 \mathrm{lb} / \mathrm{bbl}$ Bentonite suspension and a $25 \mathrm{lb} / \mathrm{bbl}$ Bentonite suspension with $20,000 \mathrm{ppm}$ sodium chloride. These base fluids were prepared in the flow loop to reduce aeration and to allow an even consistency by exposing the fluid to similar shear histories. The $\mathrm{pH}$ was constantly monitored, In the reservoir, and maintalned at $10 \pm 0.5 \mathrm{pH}$ with a sodium hydroxide solution. The flow loop is able to shear age the fluid quite effectively. The mixing period is not sufficient to age the fluid for testing. It was sufficient to evenly distribute the clays and chemicals throughout the Iiquid phase.

The mixing procedure requires shear aging the fluld for four hours at room temperature. The system pressure was maintained at 1,500 psi. 
The pressure drops were monitored as an indication of consistency. The clays and chemicals were added to the reservolr and constantly agitated with a mechanical stirrer as well as by the feed pump bypass. Fluld mixing procedures at times produced fluids which varied in consistency. This condition was reduced by determining the loop cycle time. The clays and chemicals were added evenly by dividing additions in portions of 50 grams. Two to five cycles were necessary depending on the viscous nature of the test fluld. The base fluids were originally allowed to hydrate prior to additlons of any chemicals, the hydration perfod allowed the clays sufficient time to disperse. If the chemicals were added on the second day of testing, the fluids at times were not homogeneous and required additional shear aging or exposure to heat before proper stabilization was obtained. At the end of the mixing period the fluid was displaced from the flow loop and stored in air tight containers for 16 to 20 hours.

The hydration period is primarlly for liquid-solid adsorption or chemical dispersion. The clays are sensitive to liquid contact and disperse with time. The chemicals may tend to adsorb water or may be soluble. It is also apparent that the chemical may react with the clays during this period. Reduction in dispersion of the clays resulted from the simultaneous aging of the clay and additive.

The pressure transducer capillaries were sensitive to plugging. Once the test fluid was displaced the capillary lines were flushed with fresh water and a calibration check was performed. The pump was also serviced during this period, this maintenance was necessary to reduce the possibility of pump leakage or check valve failure. If the clay 
suspenston were allowed to remain in the system the check valve efficlency of the metering pumps was reduced. The loop was then flushed for one hour at $250^{\circ} \mathrm{F}$ with a 10 to $12 \mathrm{pH}$ caustic solution. The caustic solut1on was not recirculated but dumped after periodic checks for impurities. The following day the filtration cells were installed and the corrosion coupons were weighed and placed in the corrosion cells. The test fluid is used to displace the caustic solution. The fluid was then circulated at room temperature for thirty minutes. The Baroid variable speed rheometer is used to measure the rheology at $120^{\circ} \mathrm{F}$. These data were then compared with the flow loop differential pressure drop across the $\frac{1}{4} "$ tube. If the viscosities calculated were consistently in the same range, the test was initiated. If the data were not consistant and no plugging of capillary lines was apparent, a note was recorded with the data and the test resumed. 


\section{TEST PROCEDURE}

Testing was initiated once the mixing and hydration periods were complete and fluid rheology had been checked and recorded. The temperature intervals were $250^{\circ} \mathrm{F}, 350^{\circ} \mathrm{F}, 450^{\circ} \mathrm{F}$, and $550^{\circ} \mathrm{F}$. The temperature intervals are maintalned for one hour. If the fluid remains stable after one hour of $550^{\circ} \mathrm{F}$ the test was continued until system failure or fluid degradation occurs.

Occasionally the Wallace and Tiernan triplex metering pump, limited the testing period. The slurries tested, at times, scoured the plungers and packing such that leakage occured. The pump ifquid end check valves also can produce a time limitation on the test. The fluids viscosity and solids content affected the performance of the ball check valve. The valves tend to stick either open or closed. Once a check valve became inoperative the flow rate varied requiring repair of the stuck check valve and reevaluation of the volumetric flow rate.

Absolute pressure data was recorded every 15 minutes. The absolute pressure was considered excessive if it exceeded 3,000 psi. Pumping pressure is maintained with the shear valves. The required tension to obtain the test pressure of 1,500 psi for the fluid had to be monitored because of temperature thinning effects. If the fluid exhibited temperature thinning and the pump pressure reduction was below test pressure the shear valve tension was increased. The shear valves 
were only adjusted when the loop temperature was below $350^{\circ} \mathrm{F}$. Therefore any temperature thinning effects at $450^{\circ} \mathrm{F}$ and $550^{\circ} \mathrm{F}$ and subsequent pressure reduction were not corrected. This is due mainly to safety requirements. Normally test fluid reactions above $350^{\circ} \mathrm{F}$ would cause a viscosity increase (pressure increase) due to clay flocculation or chemical reactions. Therefore over pressuring was considered as a chemical flocculation condition. The flulds are then considered to have degraded due to the test temperature. The absolute pressure was also used to correlate the differential pressure data. Two to three hundred psi fluctuations could occur at the higher temperatures $\left(350^{\circ} \mathrm{F}-\right.$ $550^{\circ} \mathrm{F}$ ).

Differential pressure drops were recorded every 15 minutes along with the absolute pressure. In certain cases of capillary tube plugging the differential pressure data reflects unreliable data. Therefore data, is eliminated where it is due positively to capillary tube plugging.

Effects of fluid vaporization problems due to fluid flashing was considered at high temperature. Vaporization developed whenever pump efficiency deterforated. The vapor pressure of water at $550^{\circ} \mathrm{F}$ is 1,050 psi. When pump pressure is reduced below this value, the liquid is partially vaporized and this reduces the reliability of the test data. The fluid may deposit solids on the tube walls restricting flow and increasing the pressure drops attained. The vapor phase eliminates the assumption of no slippage at the tube walls due to the velocity gradient of the vapor phase. Erratic pressure fluctuations are the result of the increased velocity of the vapor phase and concurrent flow of the remaining slurry. Whenever vaporization became excessive the test was terminated. 
Rheological data was recorded every 30 minutes. The Baroid variable speed rheometer measurements obtained included a range of RPM values including inftial and ten minute gel strength readings. The rheometer data was obtained at $120^{\circ} \mathrm{F}$. This data was used to obtain the plastic viscosity, yield point and apparent viscosity values. The gel strength information also served as an indication of fluid stability.

Fluid pH was recorded every 15 minutes. The returning fluid $\mathrm{pH}$ is recorded and the input fluid $\mathrm{pH}$ is maintained at $10 \pm 0.5 \mathrm{pH}$. When caustic additions became excessive, due to high temperature reactions, dry caustic was added to the reservoir in gram increments. Finally, at the $550^{\circ} \mathrm{F}$ test interval caustic additions were terminated because of the excessive additions required.

FILTRATE REDUCERS, VISCOSIFIERS, AND CHEMICAL THINNERS

Five flltrate reducers were tested. The tests were made under the same conditions as the companion tests performed by Magcobar services. The fluids tested consisted of a gum, a starch, and 3 cellulose products, CMC, HEC, and polyanionic cellulose. These fluids are primarily filtrate reducers but also act as viscosifiers. The MC and polyanionic cellulose also function as shale control inhibitors.

Drilling fluids containing guar gum are usually fresh water low solids flulds. Guar gum also works better at low $\mathrm{pH}$ and low salinity. Gypsum and calcium reduce its effectiveness. Gums chemically consist of an acid nucleus coupled with sugars. The sugar molecules require bactericidal chemicals to retard fermentation. These fluids form viscous colloldal solutions by dissolving in water. . Gums are organic carbohydrates 
derfved from organic materials. They are susceptible to temperature degradation at low values of approximately $250^{\circ} \mathrm{F}$. (Rogers ${ }^{35}$ ). Although Guar gum is less effective in high salinity water it has been used in these environments quite effectively. These Guar gum treated fluids are also good in areas where high hardness water exists.

The modified polysaccaride is a pregelatinized starch with a biocide blended into tt to retard fermentation. Its primary function is a filtrate reducer. Most starches absorb water. This reduces the free water available in the fluid and the molecules tend to lodge in filtrate pores to reduce the fluid loss. Modified polysaccaride may be used in all low solids drilling fluids. They are not susceptible to salinity or pH limitations.

The cellulose products tested are altered naturally occuring products. They are generally long chain molecules which have been polymerized. The three forms tested consisted of carboxymethyl cellulose, hydroxethyl cellulose, and polyanionic cellulose. They may lower fluid loss by plugging pores, or filming clay particles. The cellulose is insoluble in water but when treated with alkaline materials forms viscous colloidal mixtures.

MC comes in three grades definable by viscosity effects. Our tests utilized, regular viscosity grade OMC. CMC is primarily a filtrate reducer and viscosifier but also acts as a shale control inhibitor. It is sensitive to high salinity. Generally, less than $30,000 \mathrm{ppm}$ sodium chloride is desired. CMC fluids are not sensitive to the $\mathrm{pH}$ range used. The amount of salinity and ions present in the fluid influence the amount of treatment required to obtain desired fluid loss and viscosity effects. 
Normally, caustic soda and a chemical thinner are added to achieve viscosity, gel strength and pH control. Gypsum, calcium and formation shales cause contamination and reduce the effectiveness of the treatment. CMC has low fermentation degradation and may not zequire bactericides.

Eydroxyethyl cellulose, HEC, is similar to CMC but several distinctions exist. High $\mathrm{pH}$ is not desirable when using HEC. It is generally used as a viscosifier but is an excellant filtrate reducer. It is not sensitive to salinity and can be used in saturated salt water solutions. Gypsum, and calcium reduce the treatment effectiveness. Polyanionic cellulose, is a multi low solids drilling fluid additive. It acts as a filtrate reducer, shale control inhibitor and a viscosifier. It is not sensitive to salinity, $\mathrm{pH}$, gypsum, or calcium conditions.

Previous investigations have indicated that most organic filtrate reducers are not effective above $250^{\circ} \mathrm{F}$ for extended periods of time. They also must be monitored for treatment concentration because they are viscosifiers and overtreatment changes viscosity drastically. Polyanionic cellulose and HEC are similarly problematic because excessive shearing causes a dramatic viscosity change.

\section{LIGNOSULFONATES AND LIGNITES}

Two lignosulfonates and two conmercially available lignitic materials were tested. These fluids are primarily chemical thinners but have other beneficlal qualities. They generally react better at, pH values above $9.5 \mathrm{pH}$ but are efficient at any range of $\mathrm{pH}$.

Ferrochrome lignosulfonates are excellent thinners, shale 
inhibitors and fluid filtrate reducers. Gel strength is considerably reduced and controlled by these additives.

The lignosulfonates act on clays by reducing fonic attractive forces. Therefore, they effectively reduce the flocculation tendencies of bentonitic clays and are known to raise the thermal flocculation stability.

The lignite-11gnosulfonate fresh water muds tested are considered to be especially economic. They are dependent on the combined properties of Iignite to reduce filtrate loss and Ifgnosulfonate to act as a chemical thinner.

Partially refined lignites are generally recomended for high temperature stability. Hardness ions reduce base exchange capacity of lignites thereby reducing their effectiveness. Salt concęntration also reduces efficiency by reducing solubility and causing a flocculation effect.

TEST RESULTS

Three phases of fluid testing were required to determine temperature degradation characteristics of comon drilling fluid additives. The first phase consisted of a high temperature test on a water mineral oil mix to establish flow loop reliability. The second phase consisted of testing a bentonite suspension for flocculation tendencles at high temperature. The third phase involved testing of the varlous viscosifiers, filtrate reducers, and chemical thinners. 
Phase I

The pure water viscosity range was below the accuracy of the differential tranducers. The fluid as tested contained mineral oil. The oil raised the viscosity sufficiently to obtain reliable data.

Phase II

The clay water suspension tested was mixed in the loop and tested in one day. Clay hydration was minimized to reduce the viscosity and allow a more pumpable fluid.

The Bentonite slurry viscosity increased with each temperature interval. The tube viscometer and the Baroid rheometer data correlated. The rheology data did not react as expected with increased temperature. Plastic viscosity increased with increased temperature. The yield point increased to a peak, and gradually leveled off. The plastic viscosity increase and yield point characteristics could be attributed to a reduction in the liquid concentration caused by pump leakage.

Failure of the system occured between $400-450^{\circ} \mathrm{F}$ when the high temperature shear valve plugged. Actual flocculation temperature of the bentonite slurry is questionable. Failure of the shear valve was attributed to a rubber seal being installed when a viton seal was required. The seal is in contact with a woving rod which applies spring pressure to the ball and seat. The rod is required to pulsate as fluid pressure lifts the ball off of the seat. The shear valve was repaired and reinstalled on the inlet side of the water cooler. This location reduced the exposure temperature of the shear valve as much as $200^{\circ} \mathrm{F}$. No further failures were attributable to this seal. 
Pump check valve efficiency and plunger packing leakage altered the flow velocity. The complication of altered liquid-solids ratio made consistency detérmination a variable.

The test section pressure measurements were sufficient in the $1 / 4 " 0 . D$. and $3 / 8 " O . D$. tubes but the $3 / 4 " O . D$. tube pressure drops were consistently high. Reynolds number calculations indicate the fluid was in the laminar flow regime. It was concluded that channeling of the test fluid was occuring in the $3 / 4$ " tube. The reduced diameter caused by the settling tendency of the fluid caused the high pressure drops. This condition was observed in all tests involving clay slurries.

Phase III

The first chemical test consisted of a carboxymethyl cellulose slurry. The base fluid was not prehydrated but, was sheared for 2 hours in the flow loop. Test results indicated the fluid viscosity varied slightly between loop and rheometer measurements. The rheometer data indicated a reduction in plastic viscosity and a similar decrease in yield point. Tube viscometer data indicate a stable fluid up to $550^{\circ} \mathrm{F}$, that is no high viscosity. Once the temperature was raised to $550^{\circ} \mathrm{F}$ the fluid exhibited a decreasing viscosity. Certain periods were affected by pressure reductions below the vapor pressure resulting in high differential pressure fluctuations. The fluid was discolored from light gray to a brown gray. Caustic additions were excessive at the $450^{\circ} \mathrm{F}$ and $550^{\circ} \mathrm{F}$ interval. Gel strength data indicates a significant decrease after one hour of exposure to $550^{\circ} \mathrm{F}$. There was no indication of a flocculation condition except when vaporization problems and caustic additions were not 
maintained. The pump continued to cause problems with leakage and no fluid was added to the reservoir other than caustic solution. The test was terminated when vaporization problems persisted due to gradual pump failure.

Polyanionic Cellulose

Prior to initiating the test the fluid was heated to $250^{\circ} \mathrm{F}$ for one hour. This reduced the rheology data enough for measurement on the rotational viscometer. The fluid remained stable after one and a half hours at $550^{\circ} \mathrm{F}$. Caustic additions were similar to the CMC test but, caustic additions were not discontinued during this test. Flow loop viscosity decreased with temperature interval until $550^{\circ} \mathrm{F}$. The rheometer data showed a decreasing plastic viscosity and a fluctuating yield point. Tube plugging in the $3 / 8^{\prime \prime} O . D$. section capillary reduced reliability of differential pressure measurements after 15 minutes at $550^{\circ} \mathrm{F}$. At this point the pump also caused vaporization and pump leakage became excessive. The test was terminated when a thermocouple malfunctioned.

HEC

This test was terminated during the mixing period. Excessive viscosities were encountered after $0.5 \mathrm{lb} / \mathrm{bbl}$ HEC was added and exceeded viscosity limitations after one $1 \mathrm{~b} / \mathrm{bbl}$. The fluid had been exposed to $250^{\circ} \mathrm{F}$ for two hours and 45 minutes in an attempt to reduce the consistency and stabilize the fluid. Once the system plugged circulation could not be regained. 
Guar Gum

This test is the first two day test, initiated to allow pump periodic maintenance. The pump leakage generally developed after a certain time interval, approximately 4.0 hours. Since 4 hours could be used to mix and a minimum of 4 hours is required to test, the test procedure was altered. The final test period lasted for six hours in this experiment, some problems were experienced because of the check valve sticking, but the pump leakage did not become severe until after 5 hours. Some vapor pressure problems resulted from the check valves and finally caused termination of this test.

This fluid exhibited good thermal stability. Some unexplainable rheological data were exhibited probably due to the variations in pump pressure and flow velocity. The $\mathrm{pH}$ value was low after $450^{\circ} \mathrm{F}$ and required excessive amounts of caustic to regain reservoir conditions to $10 \pm 0.5 \mathrm{pH}$. The returning $\mathrm{pH}$ values recorded indicate the variation and concentration absorbed by the system. Gel strengths appear to increase but at the higher temperatures they decrease. The rotational viscometer data are significantly affected by the caustic consumption. The 600 RPM value after 15 minutes of exposure to $450^{\circ} \mathrm{F}$ exhibited an increase in viscosity, yield point and 10 minute gel strength. The $\mathrm{pH}$ value was reduced from approximately $10 \pm 1.0$ to $8.9 \mathrm{pH}$ and varied from 7.6 to $8.9 \mathrm{pH}$. The plastic viscosity fluctuates between the $250^{\circ} \mathrm{F}$ interval and the first $450^{\circ} \mathrm{F}$ measurement. Also the yield point increased from $30 \mathrm{lb} / 100 \mathrm{sq}$. ft. to $39 \mathrm{Ib} / 100 \mathrm{sq}$. ft. and then decreases to 20 and $21 \mathrm{lb} / 100 \mathrm{sq}$. ft. with returning $\mathrm{pH}$ still $10 \pm 0.5$ at $350^{\circ} \mathrm{F}$. The $450^{\circ} \mathrm{F}$ . interval causes a yield point increase to $43 \mathrm{lb} / 100 \mathrm{sq}$. ft. with returning $\mathrm{pH}$ of 8.9 . 
The $\mathrm{pH}$ decreases further when exposed to $510^{\circ} \mathrm{F}$. Plastic viscosity became unity with a yield point of $4 \mathrm{lb} / 100 \mathrm{sq}$. ft. pH consistently returned between 6.4 and $7.7 / \mathrm{pH}$. Initial and 10 minute gel strengths decreased to $1.5 \mathrm{lb} / 100 \mathrm{sq}$. ft.

\section{Modified Polysaccaride}

This fluid was difficult to mix without causing severe check valve problems. The pump check valves were easily plugged by this fluid and the $1 / 4^{\prime \prime}$ capillary tube to the $\Delta P$ transducers was later found plugged. This resulted in erratic fluctuations in differential pressure. Test section differential pressures fluctuated as much as 15.0 psi. The flow rate varied from $7.0 \mathrm{ml} / \mathrm{sec}$ to $16.0 \mathrm{ml} / \mathrm{sec}$. Pump pressures varied from 500 psi to 1,300 pst. The mixing period lasted 3.5 hours. The hydration period was 20 hours. The fluid flow appears to have been stable in the $3 / 8^{\prime \prime}$ O.D. tube. The fluid visually exhibited aeration characteristics after 30 minutes at $450^{\circ} \mathrm{F}$ of exposure. No determination as to the composition of the gaseous space could be ascertained. The fluid became a froth plugging the system and terminating the test.

The $450^{\circ} \mathrm{F}$ test interval again reflects an important characterIstic of $\mathrm{pH}$ variation, after 15 minutes of $450^{\circ} \mathrm{F}$ temperatures, the $\mathrm{pH}$ had dropped from 9.5 down to 6.4 to $6.8 \mathrm{pH}$.

The fluid remains fairly stable through the $350^{\circ} \mathrm{F}$ test interval. Plastic viscosity remained constant at $7 \mathrm{cp}$. the yield point varied slightly from 7 to $16 \mathrm{lb} / 100 \mathrm{sq}$. ft. This may be attributed to the $\mathrm{pH}$ again. This fluid exhibited a capacity for more caustic than previous fluids tested. 


\section{Ferrochrome Lignosulfonate}

This test indicated that IIgnosulfonates degrade rapidly after exposure to $450^{\circ} \mathrm{F}$. This fluid plugged the system after 45 minutes at $530^{\circ} \mathrm{F}$. The particular mixing procedure followed, as discussed under "Fluid Preparation", produced initial viscosities in the $6.5 \mathrm{cp}$ range. Higher initial viscosities were obtained in preliminary tests where the bentonite was completely hydrated before the addition of the lignosulfonates.

It should be noted that once the slurry is exposed to elevated temperatures the performance of lignosulfonates is the same (an increase in viscosity) regardless of the initial mixing sequence.

The rheological data from the returning fluid indicates instability of the ferrochrome lignosulfonate after exposure to $450^{\circ} \mathrm{F}$. The pressure indications from the loop signified the fluids were temperature sensitive immediately after exposure to $450^{\circ} \mathrm{F}$. The viscosity increase was quite significant. The difference between the two measurements, rheometer and flow loop, indicates the need of dynamic measurements. The 10 minute gel strength has significantly increased from 2 1b/100 sq. ft. ( $450^{\circ} \mathrm{F}$ after 30 minutes exposure to $401 \mathrm{~b} / 100 \mathrm{sq}$. ft. C $450^{\circ} \mathrm{F}$ after one hour. The inftial and 10 minute gel strength increased dramatically after 30 minutes of $530^{\circ} \mathrm{F}$ exposure to 13 and $108 \mathrm{lb} / 100 \mathrm{sq}$. ft. respectively. The yield point remained constant until $450^{\circ} \mathrm{F}$ when it gradually increased to $6 \mathrm{1b} / 100 \mathrm{sq}$. ft. The $550^{\circ} \mathrm{F}$ temperature interval developed a $22.4 \mathrm{lb} / 100 \mathrm{sq}$. ft. yield point. pH conditions were virtually uncontrollable after exposure to $350^{\circ} \mathrm{F}$ temperature. 
Plastic viscosity remained relatively stable until $450^{\circ} \mathrm{F}$ was attained. A slight increase in the plastic viscosity is indicated during the hour of exposure to $450^{\circ} \mathrm{F}$ temperature. The plastic viscosity then decreased when exposed to $530^{\circ} \mathrm{F}$ interval when capillary lines experienced plugging and finally the shear valve plugged. A flow loop viscosity increase was noted immediately upon exposure to $450^{\circ} \mathrm{F}$. The viscosity rose to $29 \mathrm{cp}$ within 7 minutes on the $1 / 4^{\prime \prime}$ O.D. tube. With 30 minutes of exposure the 1/4" O.D., tube viscosity was approximately $52 \mathrm{cp}$. After 45 minutes of exposure the viscosity had risen to $121 \mathrm{cp}$.

\section{Chrome Lignosulfonate}

Pump check valves were modified prior to initiating this test. It was determined that replacing the check valves prior to testing would increase test time. This partially reduced the check valve sticking tendency for this test.

Rheology data reflect a consistent fluid until the final check at $450^{\circ} \mathrm{F}$. Ten minute gel strength increased in this interval from $2 \mathrm{lb} / 100 \mathrm{sq}$. ft. to $24 \mathrm{lb} / 100 \mathrm{sq}$. ft. No significant plastic viscosity. decrease occured and only a slight increase in yield point developed. Flow loop viscosity increased after 45 minutes of exposure to $450^{\circ} \mathrm{F}$ heat. The dramatic increase experienced by the ferrochrome lignosulfonate experiment was less pronounced for chrome lignosulfonate.

This experiment was terminated due to a high degree of pressure fluctuation in all tubes. The 3/8" O.D. tube capillary line plugged and the 1/4" O.D. differential pressure fluctuated excessively.

The $\mathrm{pH}$ consumption appeared minimal in relation to the ferrochrome lignosulfonate. 
Chrome Lignosulfonate With Modified Lignite A

Random plugging of the check valves reduced the efficiency of data collected. Monitoring of absolute pressure and flow rate enabled data to be collected. This fluid responded similarly to the chrome Ifgnosulfonate with modified lignite B fluid except when exposed to $450^{\circ} \mathrm{F}$ temperature. This test was terminated due to fluid thickness and excessive pressure fluctuation.

No indication of fluid degradation may be ascertained from the rheometer. Plastic viscosity and $\mathrm{pH}$ remain fairly constant and yield point data actually decreased. Rheometer data indicated a reduced viscosity for each temperature interval similar to previous tests. No rheometer data was obtained after the viscosity increase noted in the tube viscometer at $450^{\circ} \mathrm{F}$. Returning fluid developed a froth within 15 minutes after exposure to $450^{\circ} \mathrm{F}$.

Chrome Lignosulfonate With Modified Lignite B

This test was similar to the lignosulfonates but the fluid caused a varlety of system malfunctions. The pump fluctuations due to check valve plugging reduced the reliability of the data. Flow velocity at times approached a static condition, but vaporization was not considered a problem because the pressure remained above the flash point. The test was terminated at $450^{\circ} \mathrm{F}$ after 30 minutes when the pump check valves plugged and circulation could not be regained.

The rheology data indicate a reduced viscosity with each temperature increment. The gel strength is slightly increased after exposure to $250^{\circ} \mathrm{F}$ heat for one hour, but no further increase is noted during the $350^{\circ} \mathrm{F}$ interval. 
The yield point does not reflect the viscosity increase attained in the tube viscometer. The $\mathrm{pH}$ decreases upon exposure to $350^{\circ} \mathrm{F}$. 
TABLE 2

BAROID RHEOMETER DATA

25 PPB BENTONITE

C $120^{\circ} \mathrm{SR} \simeq 511 \mathrm{sec}^{-1}$

\begin{tabular}{|c|c|c|c|c|c|}
\hline $\begin{array}{c}\text { After Temperature } \\
\text { Exposure } \\
\left({ }^{\circ} \text { Fahrenhe1t) }\right.\end{array}$ & $\begin{array}{c}\mu_{\mathrm{a}} \\
\text { Apparent } \\
\text { Viscosity } \\
\text { (Centipoise) }\end{array}$ & $\begin{array}{c}\text { PV } \\
\text { Plastic } \\
\text { Viscosity } \\
\text { (Centipoise) }\end{array}$ & $\begin{array}{c}\text { YP } \\
\text { Yield } \\
\text { Point } \\
(1 b / 100 \text { sq.ft.) }\end{array}$ & $\begin{array}{c}\text { Intt1al Gel } \\
(1 b / 100 \text { sq.ft.) }\end{array}$ & $\begin{array}{l}10 \text { Minute } \\
\text { Ge1 } \\
\text { (1b/100 sq.ft.) }\end{array}$ \\
\hline 200 & 18 & 11 & 7 & 4 & 10 \\
\hline 300 & 22 & 11.0 & 11.0 & 3 & -- \\
\hline 390 & 19.5 & 12.5 & 7 & 1.5 & 2 \\
\hline 400 & 24 & 16 & 8 & 1.5 & -- \\
\hline
\end{tabular}


TABLE 3

1/4" TUBE VISCOMETER DATA

25 PPB BENTONITE SR $\simeq 1,440 \mathrm{Sec}^{-1}$

\begin{tabular}{|c|c|c|c|c|c|c|}
\hline $\begin{array}{l}\text { Temperature } \\
\text { (OFahrenheit) }\end{array}$ & pHI & $\underset{(\mathrm{PSI})}{\Delta \mathrm{P}}$ & $\begin{array}{c}\text { Shear Stress } \\
\left(\text { dyne } / \mathrm{cm}^{2}\right)\end{array}$ & $\begin{array}{c}\mu_{e} \text { Effective } \\
\text { Viscosity } \\
\text { (Centipoise) }\end{array}$ & $\begin{array}{c}\text { Power Law } \\
n\end{array}$ & $\underset{\mathbf{k}}{\text { Coeffictents }}$ \\
\hline 200 & 9.5 & 2.6 & 206 & 14 & 0.569 & 2.98 \\
\hline 350 & 9.5 & 7.0 & 555 & 38 & 0.077 & 284 \\
\hline 445 & - & 18.0 & 1,427 & 99 & 0.046 & 939 \\
\hline
\end{tabular}




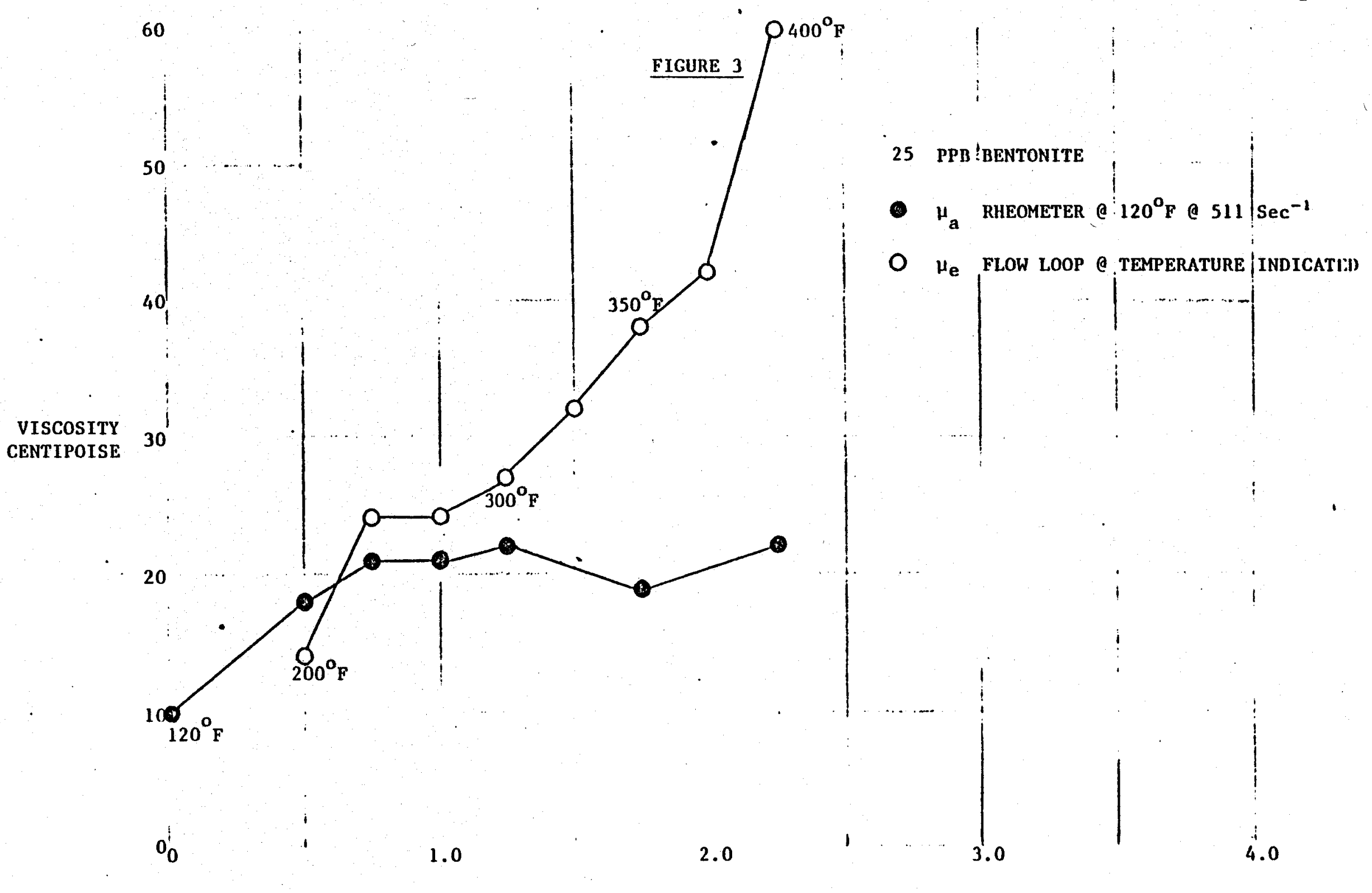

TIME HOURS 

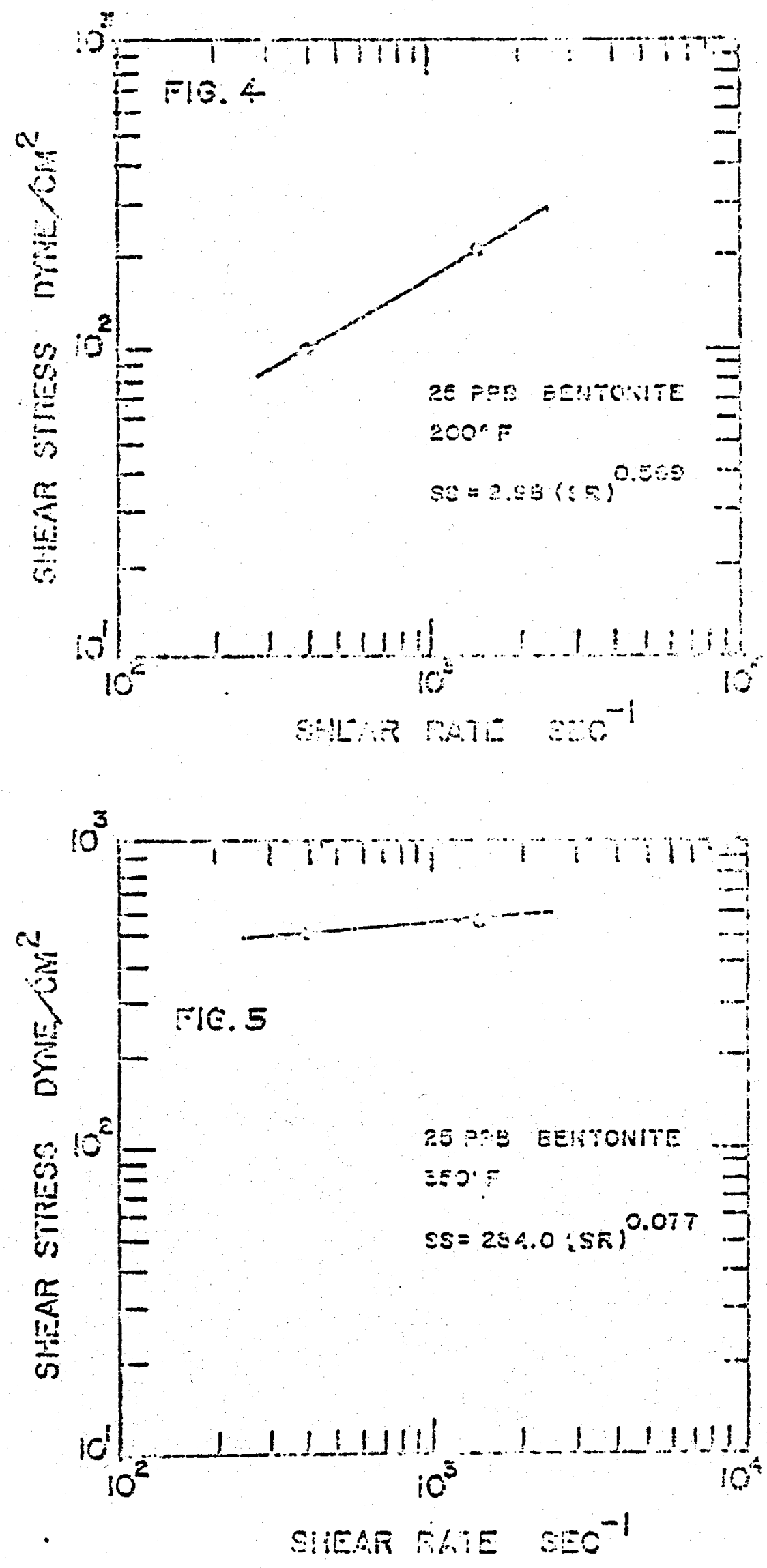


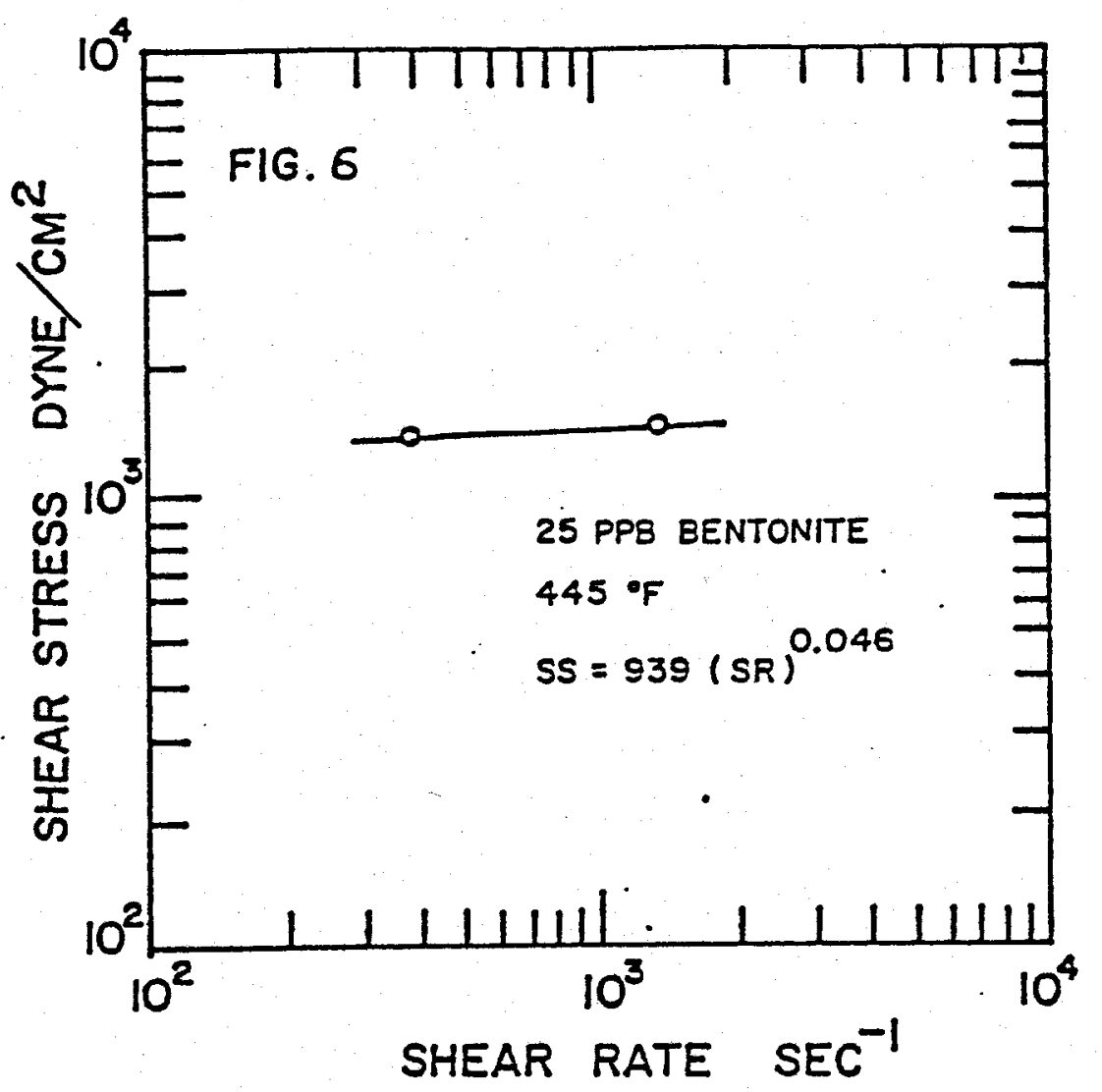


TABLE 4

BAROID RHEOMETER DATA

$2 \mathrm{PPB} \mathrm{CMC} \quad$ C $120^{\circ} \mathrm{F} \mathrm{SR} \simeq 511 \mathrm{sec}^{-1}$

\begin{tabular}{|c|c|c|c|c|c|}
\hline $\begin{array}{c}\text { Af ter Temperature } \\
\text { Exposure } \\
\text { ( }{ }^{\circ} \text { Fahrenheit) }\end{array}$ & $\begin{array}{c}\mu_{a} \\
\text { Apparent } \\
\text { Viscosity } \\
\text { (Centipoise) }\end{array}$ & $\begin{array}{c}\text { PV } \\
\text { Plastic } \\
\text { Viscosity } \\
\text { (Centipoise) }\end{array}$ & $\begin{array}{c}\text { YP } \\
\text { Yield } \\
\text { Point } \\
(1 b / 100 \text { sq.ft.) }\end{array}$ & $\begin{array}{l}\text { Intitial Ge1 } \\
(1 \mathrm{~b} / 100 \mathrm{sq} . \mathrm{ft} .)\end{array}$ & $\begin{array}{l}10 \text { Minute } \\
\text { Gel } \\
(1 b / 100 \text { sq.ft.) }\end{array}$ \\
\hline 75 & 14 & 4 & 10 & 20 & 30 \\
\hline 120 & 36 & 18 & 14 & 15 & 41 \\
\hline 250 & 23 & 11 & 12 & 5 & 28 \\
\hline 350 & 13 & 10 & 3 & 2 & 12 \\
\hline 450 & 10 & 5 & 5 & 3 & 2 \\
\hline 550 & 8 & 2 & 6 & 10 & 15 \\
\hline 550 & $\cdot 4$ & 1 & 3 & 2 & 2 \\
\hline
\end{tabular}


TABLE 5

1/4" TUBE VISCOMETER DATA

$2 \mathrm{PPB} \mathrm{CMC} \quad \mathrm{SR} \cong 820 \mathrm{sec}^{-1}$

\begin{tabular}{|c|c|c|c|c|c|c|}
\hline $\begin{array}{c}\text { Temperature } \\
\text { (OFahrenhe1t) }\end{array}$ & pH & $\underset{(\mathrm{Ps} 1)}{\Delta \mathrm{P}}$ & $\begin{array}{c}\text { Shear Stress } \\
\left(\text { dyne } / \mathrm{cm}^{2}\right)\end{array}$ & $\begin{array}{c}\mu_{e} \text { Effective } \\
V \text { iscosity } \\
\text { (Centipotse) }\end{array}$ & $\begin{array}{c}\text { Power Law } \\
\mathbf{n}\end{array}$ & $\begin{array}{c}\text { Coeffictents } \\
k\end{array}$ \\
\hline 75 & 9.9 & 2.8 & 222 & 27 & 0.731 & 1.547 \\
\hline 250 & 9.5 & 1.7 & 134 & 16 & 0.246 & 22.470 \\
\hline 350 & 9.5 & 2.0 & 158 & 19 & 0.154 & 49.370 \\
\hline 450 & 8.7 & 1.8 & 142 & 17 & 0.123 & 55.120 \\
\hline 550 & 8.2 & 0.6 & 47 & 5 & 1.046 & 0.042 \\
\hline
\end{tabular}




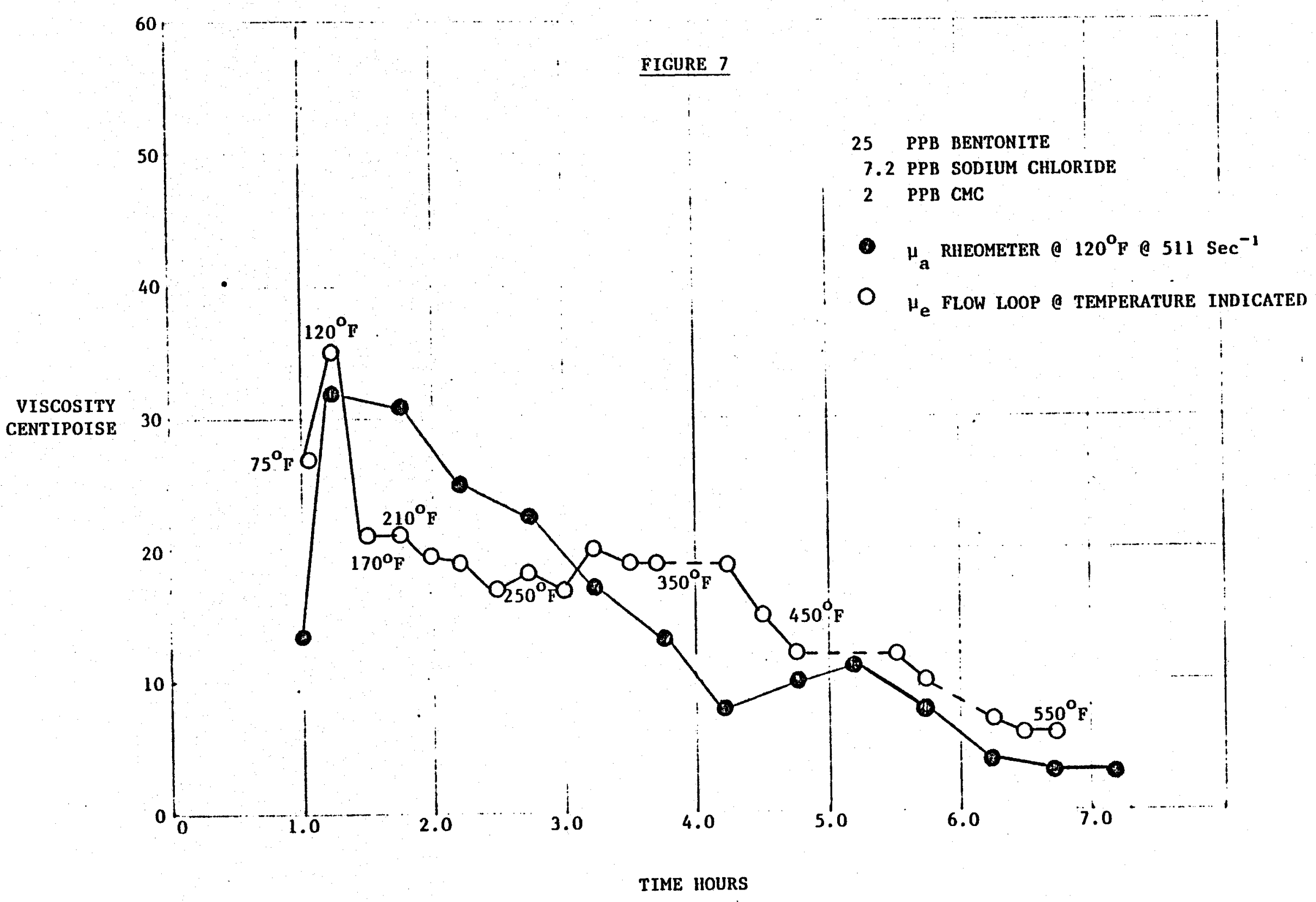

----DASIILD LINE INDICATED PERIODS OF ERRATIC PRESSURE SURGES 

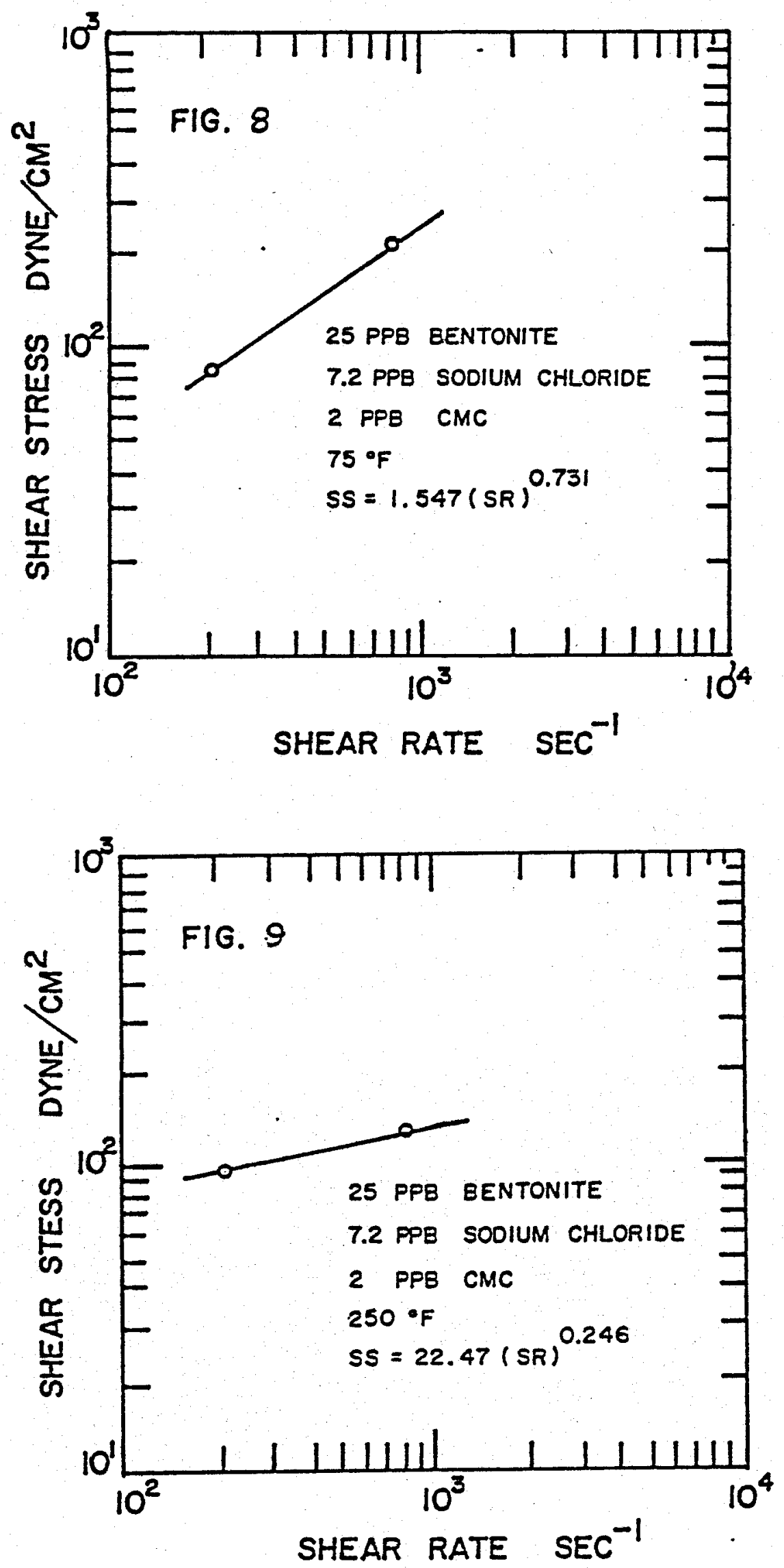

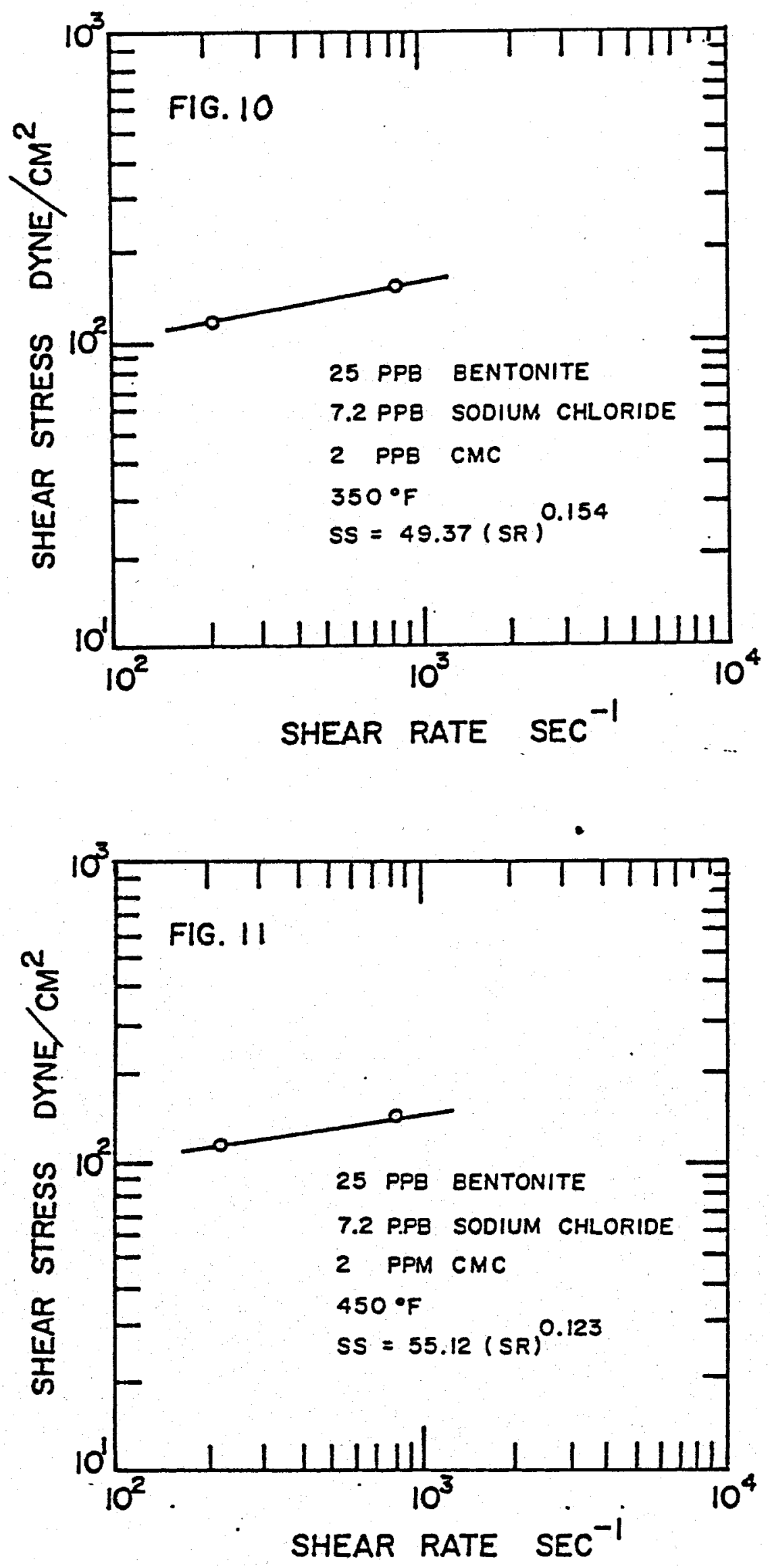


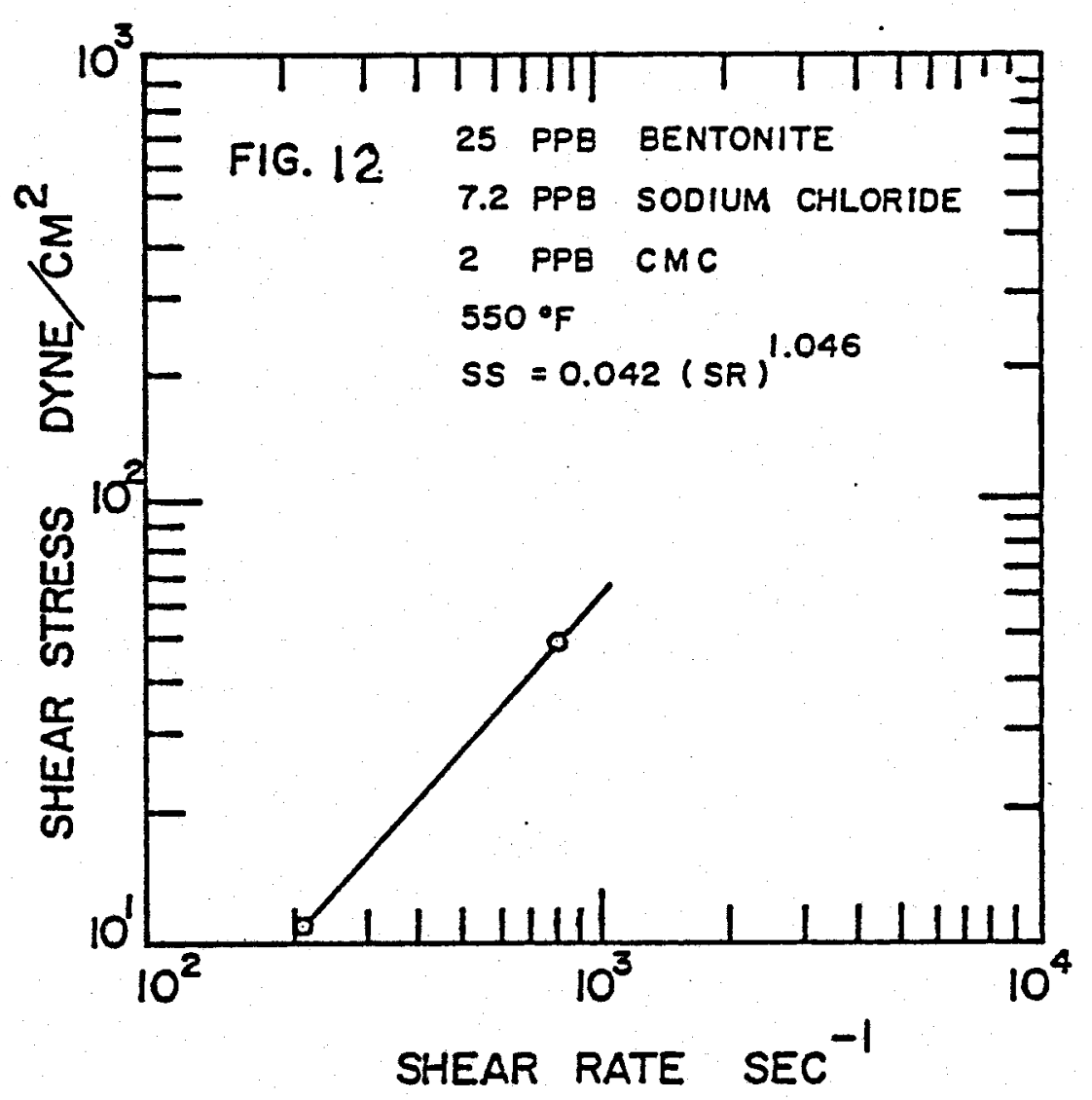


TABLE 6

BAROID RHEOMETER DATA

$2 \mathrm{PPB}$ POLYANIONIC CELluLOSE $\quad$ C $120^{\circ} \mathrm{F} \mathrm{SR} \simeq 511 \mathrm{Sec}^{-1}$

\begin{tabular}{|c|c|c|c|c|c|}
\hline $\begin{array}{c}\text { After Temperature } \\
\text { Exposure } \\
\text { (oFahrenheit) }\end{array}$ & $\begin{array}{c}\mu_{\mathrm{a}} \\
\text { Apparent } \\
V \text { iscosity } \\
\text { (Centipoise) }\end{array}$ & $\begin{array}{c}\text { PV } \\
\text { Plastic } \\
\text { Viscosity } \\
\text { (Centipoise) }\end{array}$ & $\begin{array}{c}\text { YP } \\
\text { Yleld } \\
\text { Point } \\
(1 b / 100 \text { sq.ft.) }\end{array}$ & $\begin{array}{c}\text { InftIal Gel } \\
(1 \mathrm{~b} / 100 \mathrm{sq} . \mathrm{ft} .)\end{array}$ & $\begin{array}{c}10 \text { Minute } \\
\text { Gel } \\
(1 \mathrm{~b} / 100 \mathrm{sq} . \mathrm{ft} .)\end{array}$ \\
\hline 350 & 70 & 23 & 47 & 155 & 230 \\
\hline 450 & 34 & 1 & 33 & 40 & 85 \\
\hline 550 & 7 & 3 & 4 & 8 & 18 \\
\hline
\end{tabular}


TABLE 7

1/4" TUBE VISCOMETER DATA

2 PPB POLYANIONIC CELLULOSE

$\mathrm{SR} \simeq 1,147 \mathrm{Sec}^{-1}$

\begin{tabular}{|c|c|c|c|c|c|c|}
\hline $\begin{array}{l}\text { Temperature } \\
\left({ }^{\circ} \text { Fahrenheit }\right)\end{array}$ & $\mathbf{p H}$ & $\begin{array}{c}\Delta \mathrm{P} \\
(\text { Ps1) }\end{array}$ & $\begin{array}{c}\text { Shear Stress } \\
\left(\text { dyne } / \mathrm{cm}^{2}\right)\end{array}$ & $\begin{array}{c}\mu_{e} \text { Effective } \\
\text { Viscosity } \\
\text { (Centipoise) }\end{array}$ & $\begin{array}{c}\text { Power Law } \\
\text { n }\end{array}$ & $\underset{k}{\text { Coefficients }}$ \\
\hline 75 & 8.7 & 4.5 & 356 & 31 & 0.407 & 17.800 \\
\hline 250 & 9.6 & 14.0 & 1,110 & 97 & 0.223 & 200.600 \\
\hline 450 & 7.5 & 1.8 & 143 & 12 & 0.200 & 30.410 \\
\hline 550 & 7.6 & 1.6 & 127 & 11 & 0.561 & 2.206 \\
\hline
\end{tabular}




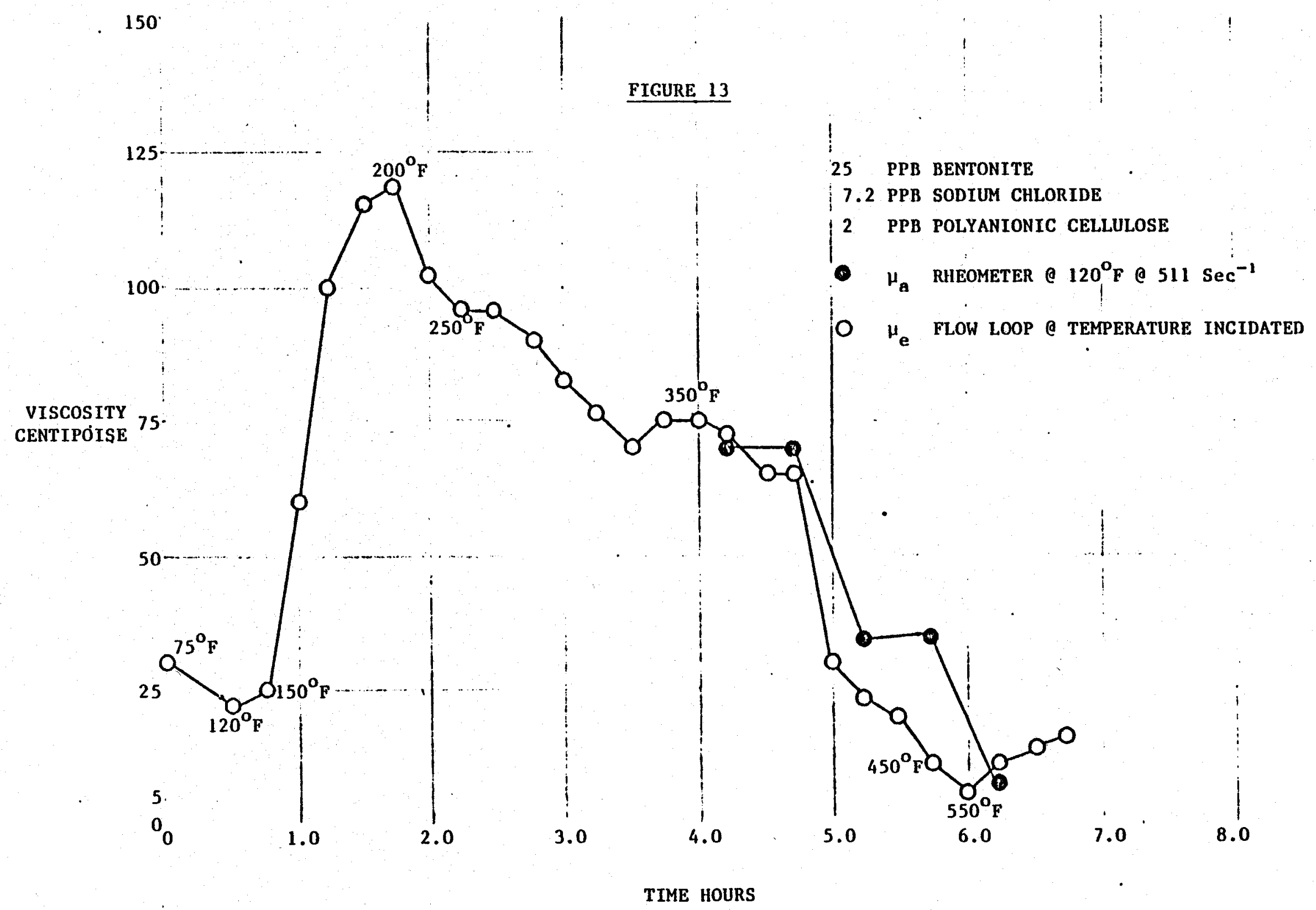



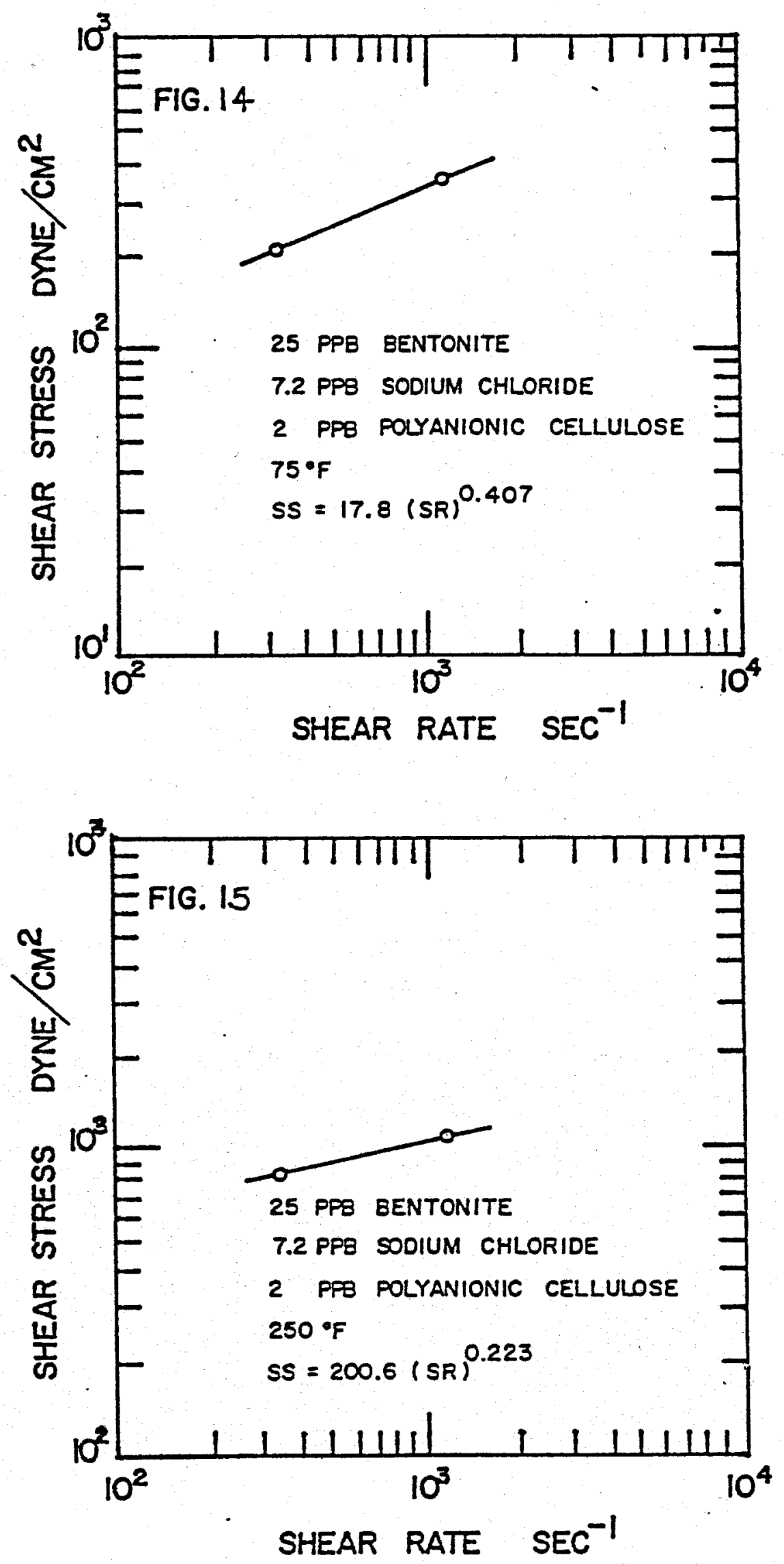

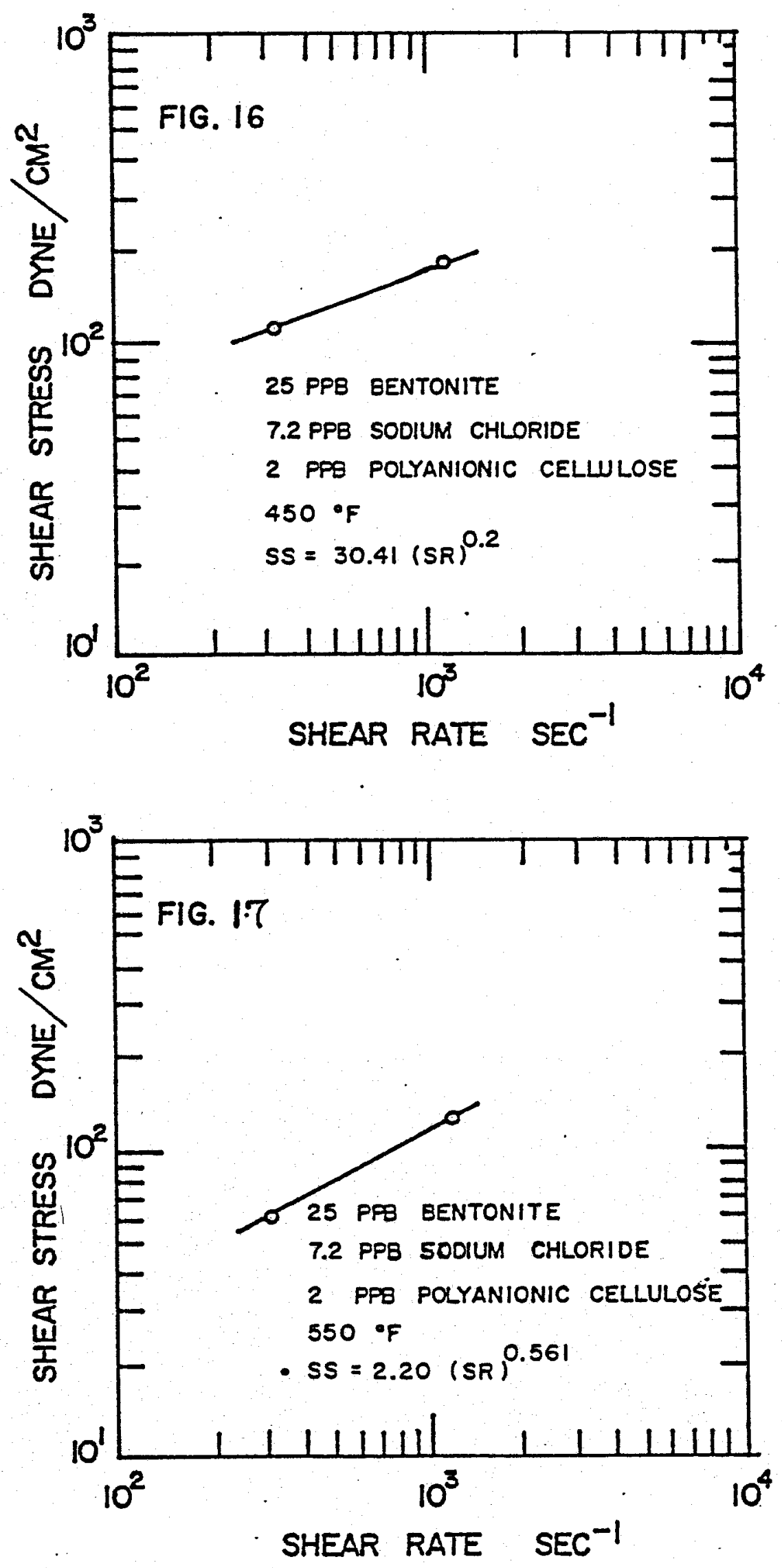
TABLE 8

BAROID RHEOMETER DATA

$2 \mathrm{PPB}$ GUAR GUM $\quad$ C $120^{\circ} \mathrm{F} \mathrm{SR} \simeq 511 \mathrm{sec}^{-1}$

\begin{tabular}{|c|c|c|c|c|c|}
\hline $\begin{array}{c}\text { After Temperature } \\
\text { Exposure } \\
\left({ }^{\circ} \text { Fahrenheit }\right)\end{array}$ & $\begin{array}{c}\mu_{\mathrm{a}} \\
\text { Apparent } \\
\text { Viscosity } \\
\text { (Centipoise) }\end{array}$ & $\begin{array}{c}\text { PV } \\
\text { Plastic } \\
\text { Viscosity } \\
\text { (Centipolse) }\end{array}$ & $\begin{array}{c}\text { YP } \\
\text { Yleld } \\
\text { Point } \\
\text { (1b/100 sq.ft.) }\end{array}$ & $\begin{array}{l}\text { Inttial Gel } \\
(1 b / 100 \text { sq.ft.) }\end{array}$ & $\begin{array}{l}10 \text { Minute } \\
\text { Gel } \\
\text { (1b/100 sq.ft.) }\end{array}$ \\
\hline 75 & 41 & 11 & 30 & 17 & 22 \\
\hline 250 & 50 & 11 & 39 & 17 & 30 \\
\hline 350 & 24 & 3 & 21 & 23 & 65 \\
\hline 450 & 21.5 & 5 & 16.5 & 11 & 11 \\
\hline 510 & 5 & 1 & 4 & 3 & 3.5 \\
\hline
\end{tabular}


TABLE 9

1/4" TUBE VISCOMETER DATA

2 PPB GUAR GUM

$S R \simeq 1,224 \mathrm{Sec}^{-1}$

\begin{tabular}{|c|c|c|c|c|c|c|}
\hline $\begin{array}{l}\text { Temperature } \\
\text { ( }{ }^{\text {Fahrenheft })}\end{array}$ & pll & $\underset{(\text { Ps1) }}{\Delta \mathbf{P}}$ & $\begin{array}{c}\text { Shear Stress } \\
\left(\text { dyne } / \mathrm{cm}^{2}\right)\end{array}$ & $\begin{array}{c}\mu_{\mathrm{e}} \text { Effective } \\
\mathrm{v} 1 \text { scosity } \\
\text { (Centipoise) }\end{array}$ & $\begin{array}{c}\text { Power Law } \\
\mathbf{n}\end{array}$ & $\begin{array}{c}\text { Coefficients } \\
k\end{array}$ \\
\hline 75 & 9.7 & 4.8 & 380 & 31 & 0.670 & 2.99 \\
\hline 250 & 10.6 & 3.4 & 270 & 22 & 0.075 & 142.5 \\
\hline 350 & 10.4 & 3.2 & 254 & 20 & 0.486 & 7.15 \\
\hline 450 & 7.7 & 0.6 & 47 & 4 & 0.583 & 0.676 \\
\hline 510 & 6.5 & 2.0 & 158 & 12 & $\cdots$ & $\cdots$ \\
\hline
\end{tabular}




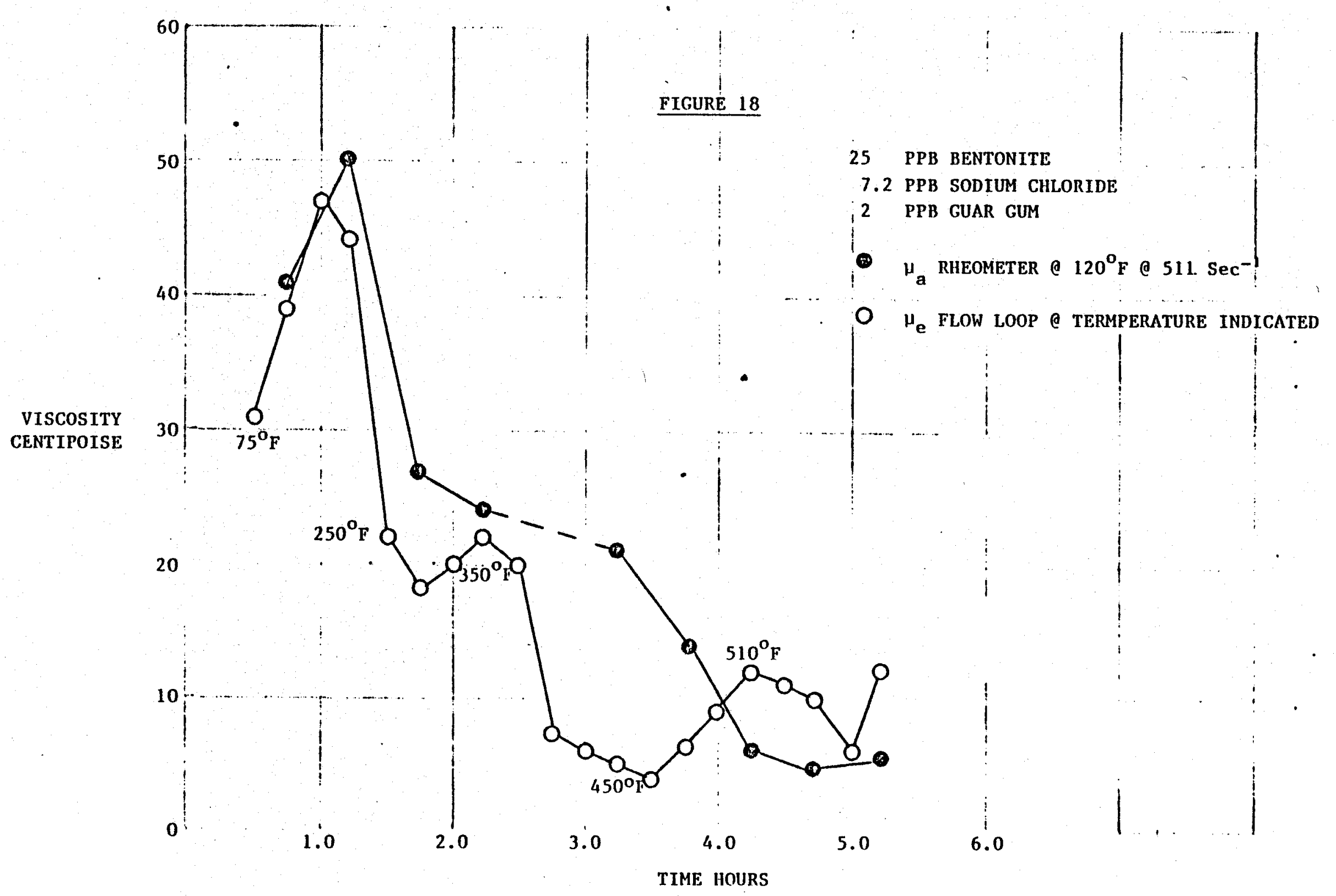



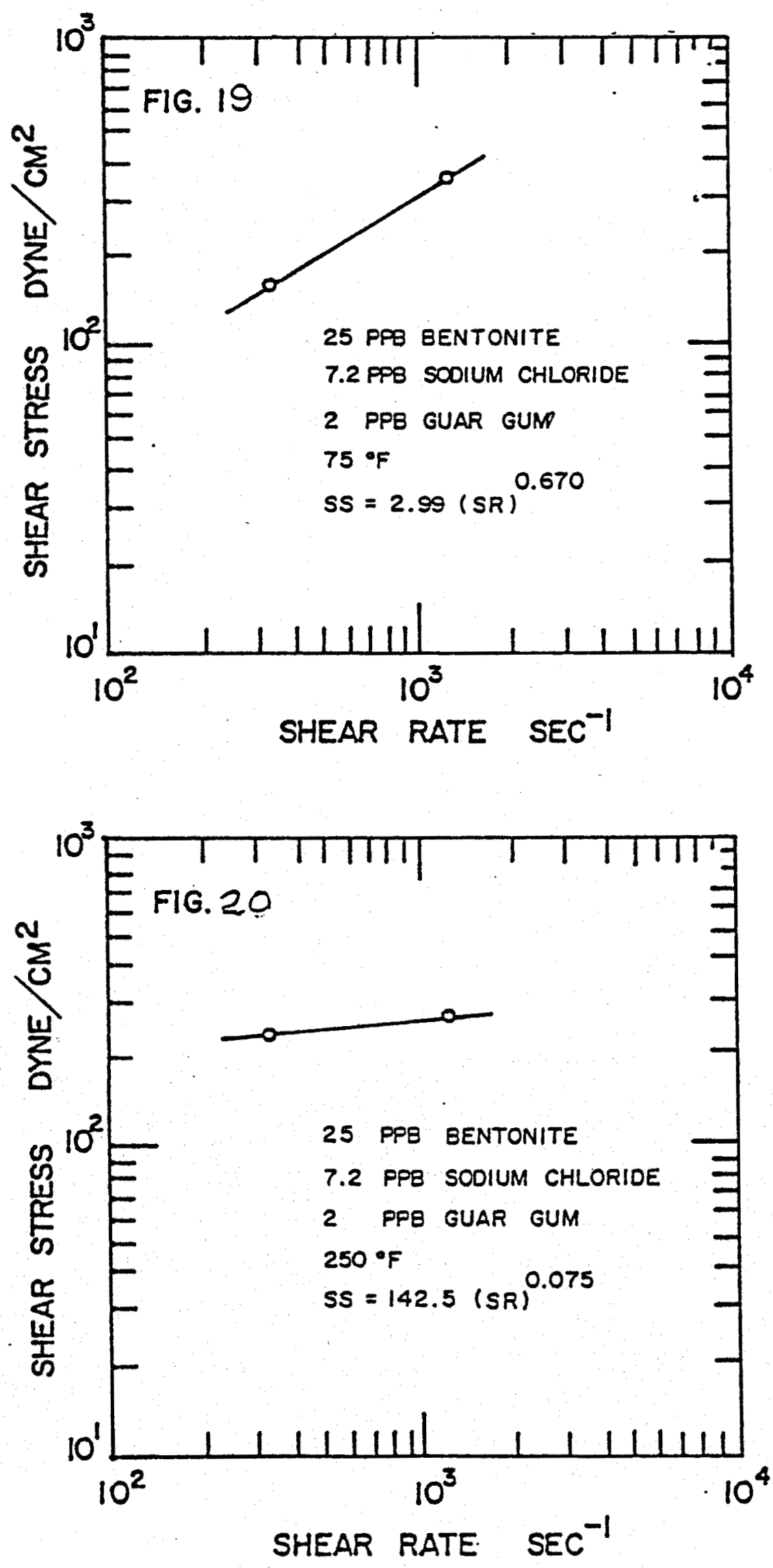

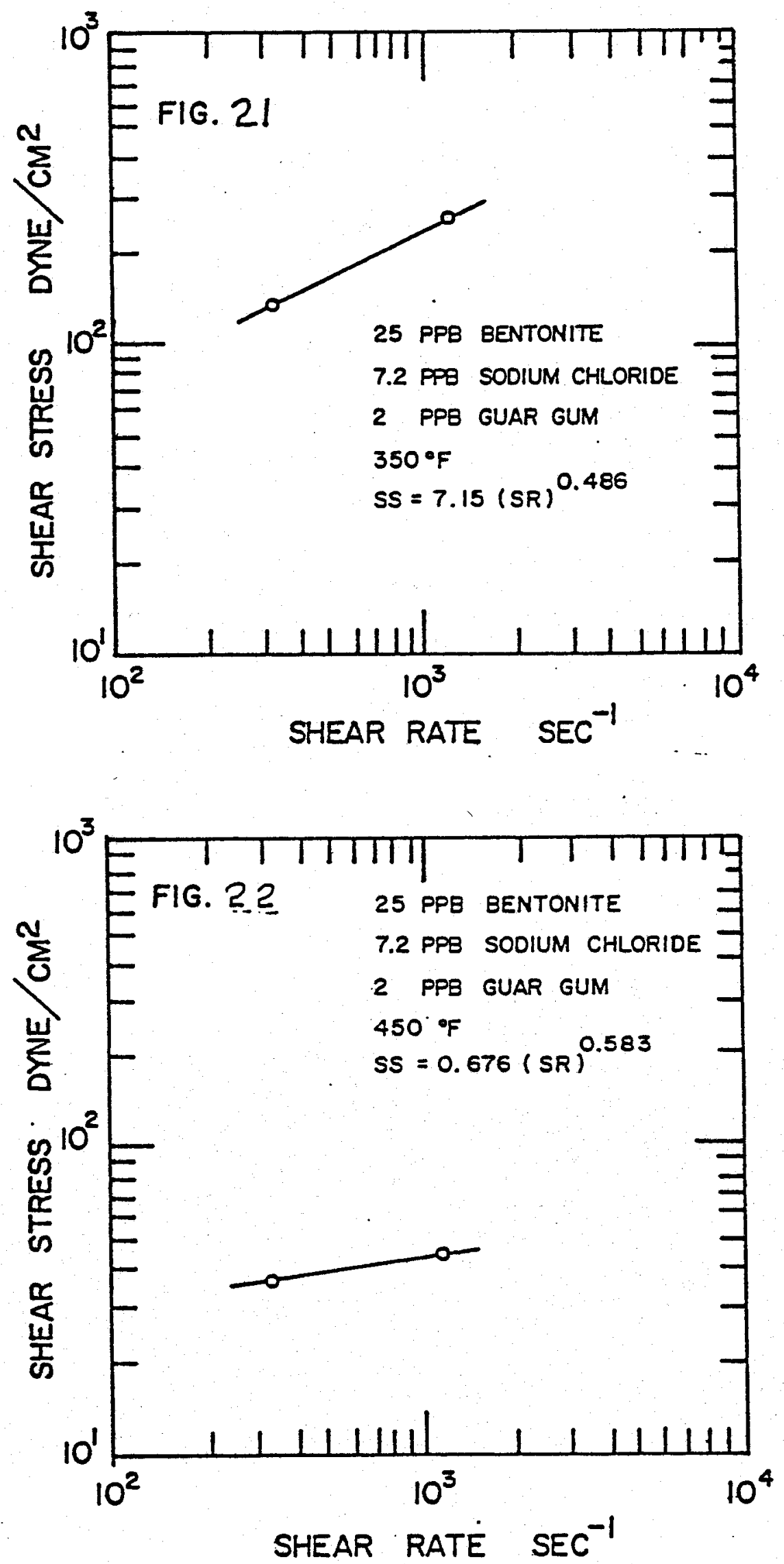
TABLE 10

BAROID RHEOMETER DATA

$5 \mathrm{PPB}$ Mod. Polysaccaride $\quad$ e $120^{\circ} \mathrm{F} \mathrm{SR} \simeq 511 \mathrm{Sec}^{-1}$

\begin{tabular}{|c|c|c|c|c|c|}
\hline $\begin{array}{c}\text { After Temperature } \\
\text { Exposure } \\
\text { ( }{ }^{\circ} \text { Fahrenhe1t) }\end{array}$ & $\begin{array}{c}\mu_{a} \\
\text { Apparent } \\
V \text { iscosity } \\
\text { (Centipolse) }\end{array}$ & $\begin{array}{c}\text { PV } \\
\text { Plastic } \\
\text { Viscosity } \\
\text { (Centipoise) }\end{array}$ & $\begin{array}{c}\text { YP } \\
\text { Yleld } \\
\text { Point } \\
(1 \mathrm{lb} / 100 \text { sq.ft.) }\end{array}$ & $\begin{array}{l}\text { Initial Gel } \\
(1 \mathrm{~b} / 100 \mathrm{sq.ft})\end{array}$ & $\begin{array}{l}10 \text { Minute } \\
\text { Gel } \\
\text { (1b/100 sq.ft.) }\end{array}$ \\
\hline 75 & 89 & 12 & 77 & 41 & 66 \\
\hline 250 & 17 & 8 & 9 & 5.5 & - 50 \\
\hline 350 & 14 & 7 & 7 & 6 & 62 \\
\hline 450 & 48 & 4 & 44 & 25 & 20 \\
\hline
\end{tabular}


TABLE 11

3/8" TUBE VISCOMETER DATA

5 PPB Mod. Polysaccaride $S R=820 \mathrm{Sec}^{-1}$

\begin{tabular}{|c|c|c|c|c|c|c|}
\hline $\begin{array}{l}\text { Temperature } \\
\text { ( } \mathrm{F} \text { Fahrenheit) }\end{array}$ & $\mathrm{pH}$ & $\begin{array}{c}\Delta \mathrm{P} \\
(\mathrm{Ps} 1)\end{array}$ & $\begin{array}{c}\text { Shear Stress } \\
\left(\text { dyne } / \mathrm{cm}^{2}\right)\end{array}$ & $\begin{array}{c}\mu_{e} \text { Effective } \\
V \text { iscosity } \\
\text { (Centipoise) }\end{array}$ & $\begin{array}{c}\text { Power Law } \\
\mathbf{n}\end{array}$ & $\underset{k}{\text { Coefficients }}$ \\
\hline 75 & 9.7 & 7.0 & 555 & 67 & 0.221 & 102.2 \\
\hline 250 & 9.5 & 4.8 & 380 & 46 & 0.265 & 51.4 \\
\hline 450 & 6.8 & 2.0 & 159 & 19 & $\cdots$ & $-\cdots$ \\
\hline
\end{tabular}




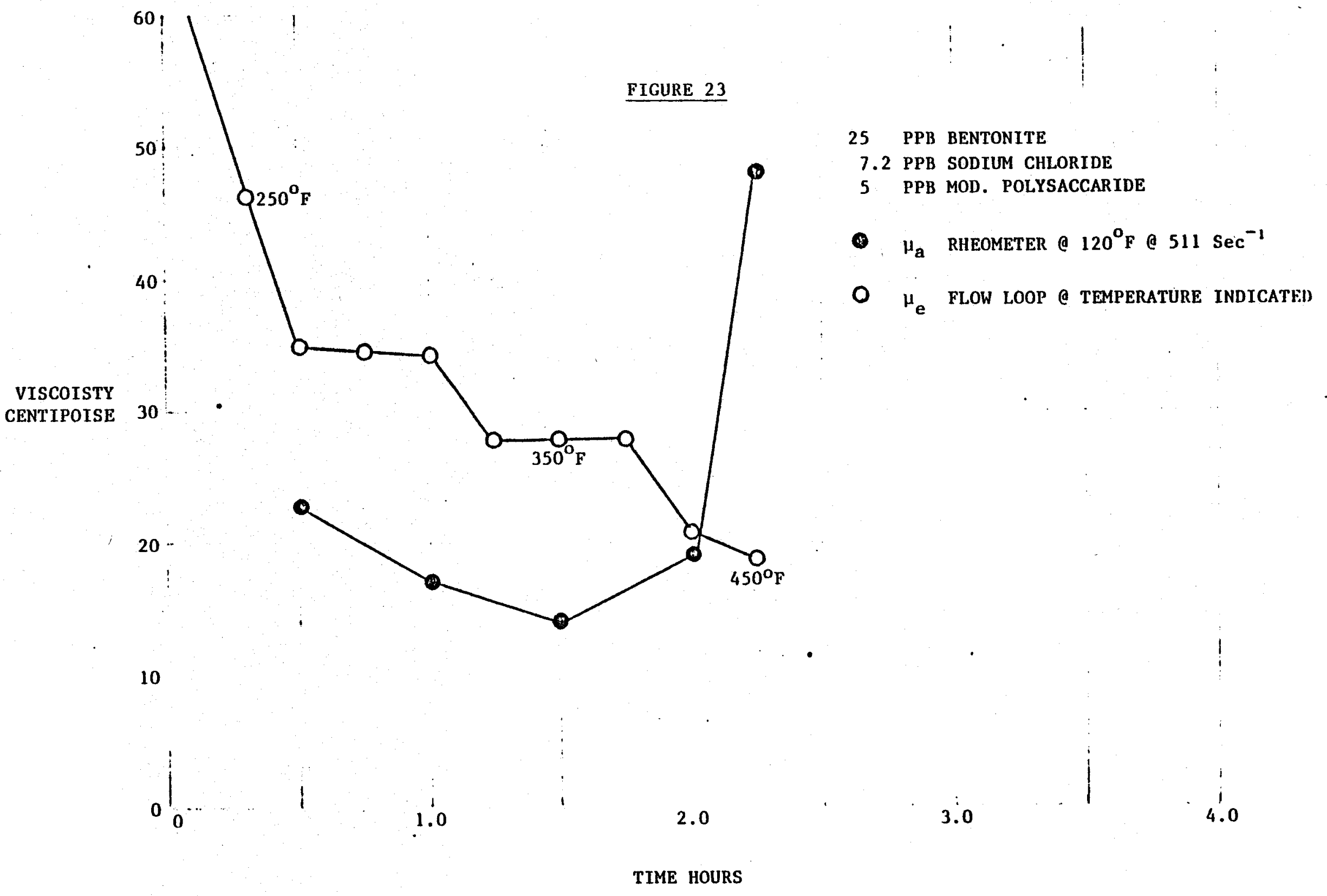



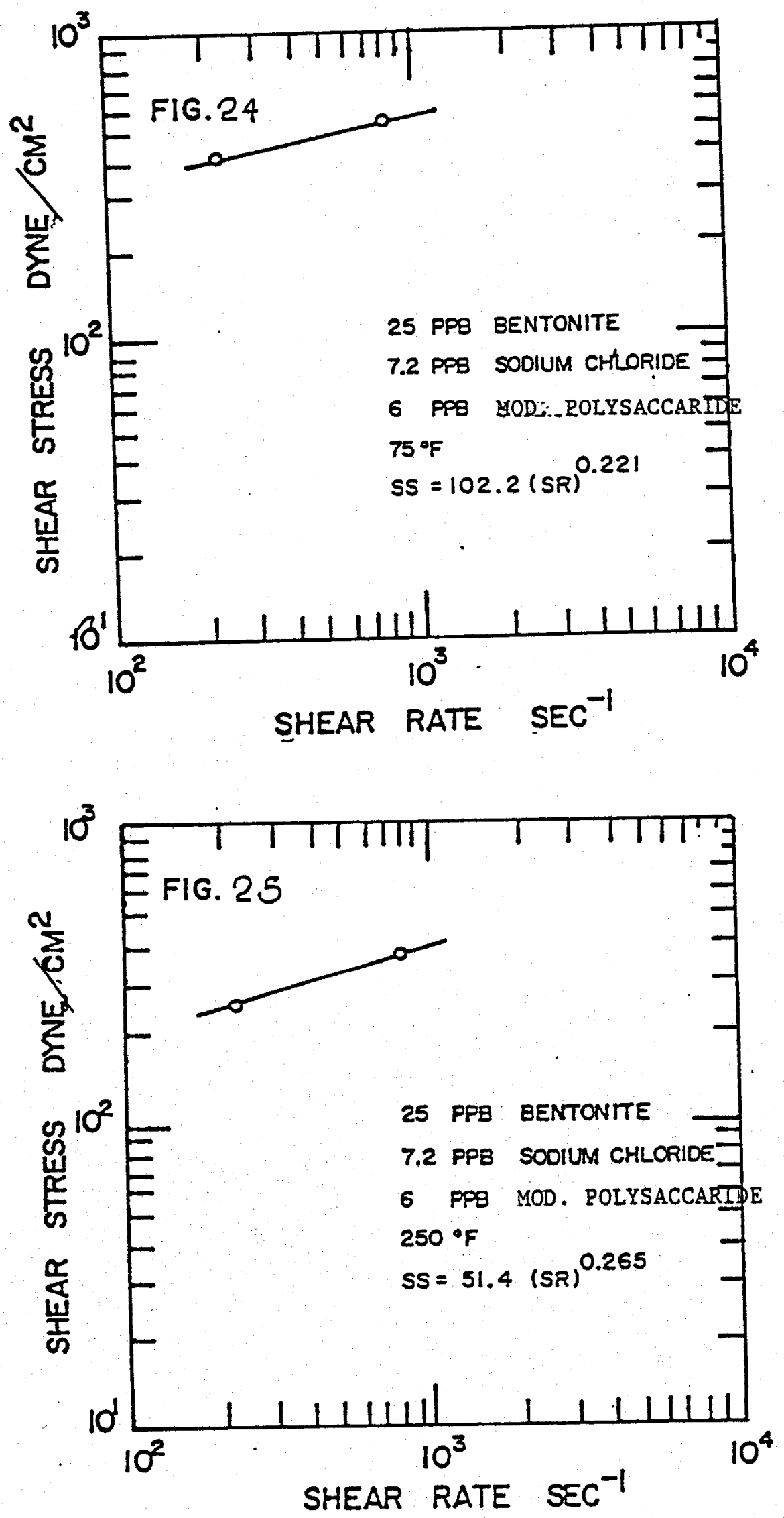
TABLE 12

BAROID RHEOMETER DATA

6 PPB FERROCHROME LIGNOSULFONATE

(e) $120^{\circ} \mathrm{F} \mathrm{SR} \simeq 511 \mathrm{Sec}^{-1}$

\begin{tabular}{|c|c|c|c|c|c|}
\hline $\begin{array}{c}\text { After Temperature } \\
\text { Exposure } \\
\text { ( Fahrenheit) }\end{array}$ & $\begin{array}{c}\mu_{a} \\
\text { Apparent } \\
\text { Viscosity } \\
\text { (Centipoise) }\end{array}$ & $\begin{array}{c}\text { PV } \\
\text { Plastic } \\
\text { Viscosity } \\
\text { (Centipotse) }\end{array}$ & $\begin{array}{c}\text { YP } \\
\text { Yleld } \\
\text { Point } \\
(1 \mathrm{l} / 100 \text { sq.ft.) }\end{array}$ & $\begin{array}{c}\text { Inttial Gel } \\
(1 b / 100 \text { sq.ft.) }\end{array}$ & $\begin{array}{c}10 \text { Minute } \\
\text { Gel } \\
(1 b / 100 \text { sq.ft.) }\end{array}$ \\
\hline 75 & 6 & 5 & 1 & 1 & 1 \\
\hline 250 & 6.5 & 4.5 & 2 & 1 & 1 \\
\hline 350 & 6.5 & 5 & 1.5 & 1 & 1 \\
\hline 450 & 12.6 & 6.5 & 6 & 1.5 & 40 \\
\hline 530 & 25.5 & 3.0 & 22.5 & 13 & 108 \\
\hline
\end{tabular}


TABLE 13

1/4" TUBE VISCOMETER DATA

6 PPB FERROCHROME LIGNOSULFONATE

\begin{tabular}{|c|c|c|c|c|c|c|}
\hline $\begin{array}{l}\text { Temperature } \\
\text { ('Fahrentelt) }\end{array}$ & $\mathrm{pH}$ & $\begin{array}{c}\Delta \mathrm{P} \\
(\mathrm{Psi})\end{array}$ & $\begin{array}{c}\text { Shear Stress } \\
\left(\text { dyne } / \mathrm{cm}^{2}\right)\end{array}$ & $\begin{array}{c}\mu_{e} \text { Effective } \\
\text { Viscosity } \\
\text { (Centipoise) }\end{array}$ & $\underset{\mathbf{n}}{\text { Power Law }}$ & $\underset{k}{\text { Coeffictents }}$ \\
\hline 350 & 8.8 & 0.25 & 19.8 & 1.6 & $-\cdots$ & -- \\
\hline 450 & 7.0 & 8.5 & 674.0 & 56.0 & -- & -- \\
\hline
\end{tabular}

$S R \simeq 1,210 \mathrm{Sec}^{-1}$ 
1

60

FIGURE 26

25 PPB BENTONITE

50

PPB FERROCHROME LIGNOSULFONATE

- $\mu_{a}$ RHEOMEter e $120^{\circ} \mathrm{F}$ @ $511 \mathrm{Sec}^{-1}$

O $\mu_{e}$ FLOW LOOP O TEMPERATURE INDICATED

40

VISCOSITY
CENTIPOOISE

20
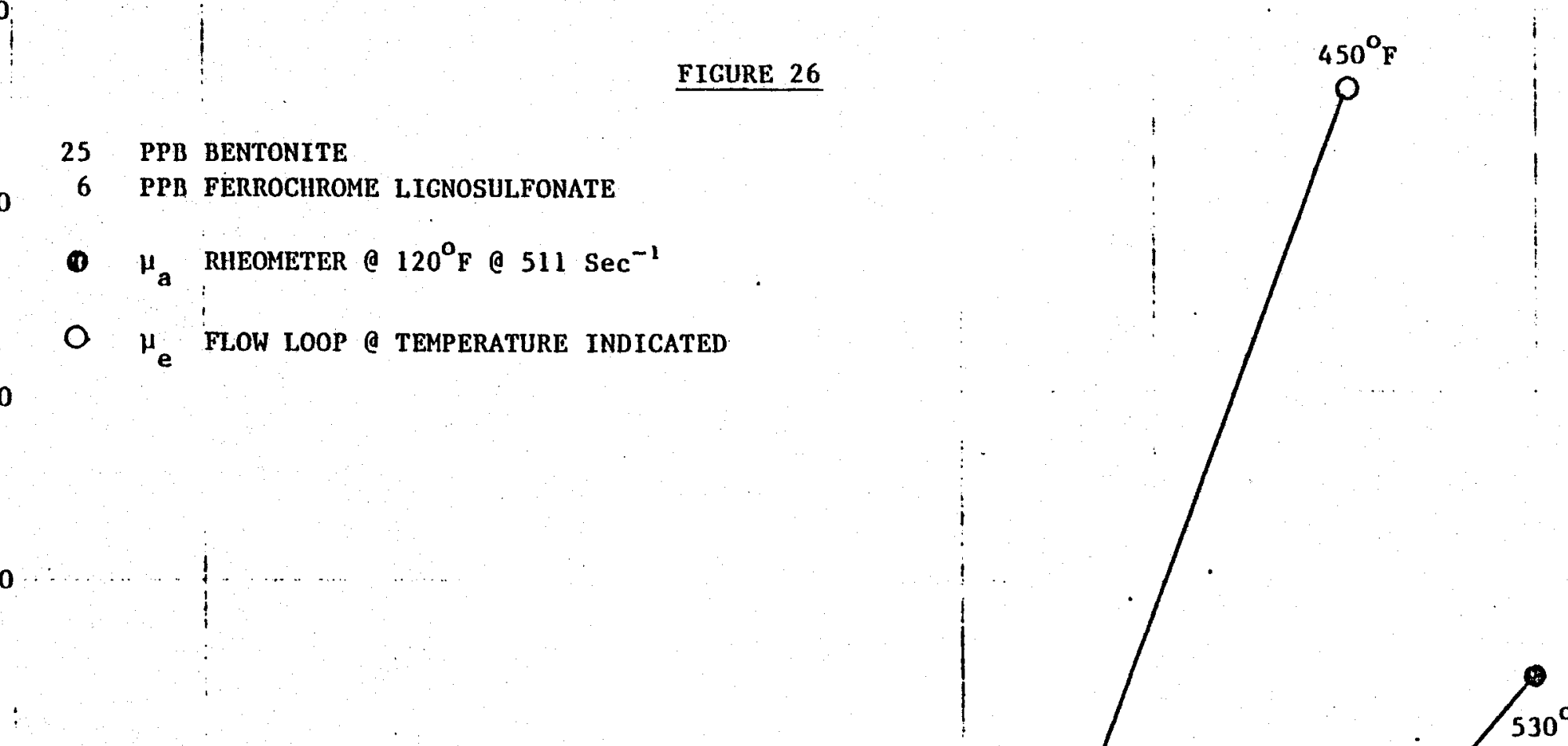

CENT IPOTE 30

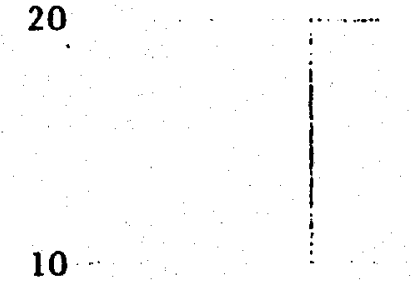

10
$\vdots$
$\vdots$
0

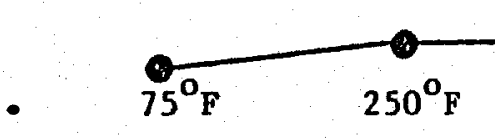

1.0

1.0

2.0

3.0

4.0

TIME HOURS 
TABLE 14

BAROID RHEOMETER DATA

$6 \mathrm{PPB}$ CHROME LIGNOSULFONATE $\quad$ c $120^{\circ} \mathrm{F} \mathrm{SR} \simeq 511 \mathrm{sec}^{-1}$

\begin{tabular}{|c|c|c|c|c|c|}
\hline $\begin{array}{c}\text { After Temperature } \\
\text { Exposure } \\
\text { ( Fahrenhe1t) }\end{array}$ & $\begin{array}{c}\mu_{\mathrm{a}} \\
\text { Apparent } \\
\text { Viscosity } \\
\text { (Centipoise) }\end{array}$ & $\begin{array}{c}\text { PV } \\
\text { Plastic } \\
\text { Viscosity } \\
\text { (Centipoise) }\end{array}$ & $\begin{array}{c}\text { YP } \\
\text { Yield } \\
\text { Point } \\
(1 b / 100 \text { sq.ft.) }\end{array}$ & $\begin{array}{l}\text { Inttial Gel } \\
(1 \mathrm{~b} / 100 \text { sq.ft.) }\end{array}$ & $\begin{array}{c}10 \text { Minute } \\
\text { Gel } \\
(1 \mathrm{~b} / 100 \text { sq.ft.) }\end{array}$ \\
\hline 75 & 12.5 & 9.5 & 3 & 1 & 1 \\
\hline 250 & 13.0 & 8 & 5 & 1 & 1.5 \\
\hline 350 & 11.0 & 7 & 4 & 1.5 & 1.5 \\
\hline 450 & 14.5 & 6.5 & 8 & 3 & 24 \\
\hline
\end{tabular}


TABLE 15

1/4" TUBE VISCOMETER DATA

6 PPB CHROME LIGNOSULFONATE

$\mathrm{SR} \simeq 1,420 \mathrm{Sec}^{-1}$

\begin{tabular}{|c|c|c|c|c|c|c|}
\hline $\begin{array}{l}\text { Temperature } \\
\text { (oFahrenheit) }\end{array}$ & pH & $\begin{array}{c}\Delta P \\
(\text { Psi) }\end{array}$ & $\begin{array}{c}\text { Shear Stress } \\
\left(\text { dyne } / \mathrm{cm}^{2}\right)\end{array}$ & $\begin{array}{c}\mu_{e} \text { Effective } \\
\text { Viscosity } \\
\text { (Centipoise) }\end{array}$ & $\begin{array}{c}\text { Power Law } \\
n\end{array}$ & $\begin{array}{c}\text { Coefficients } \\
k\end{array}$ \\
\hline 450 & 7.3 & 1.1 & 92.7 & 6 & 0.336 & 8.09 \\
\hline
\end{tabular}


$60 \cdots$

\section{FIGURE 27}

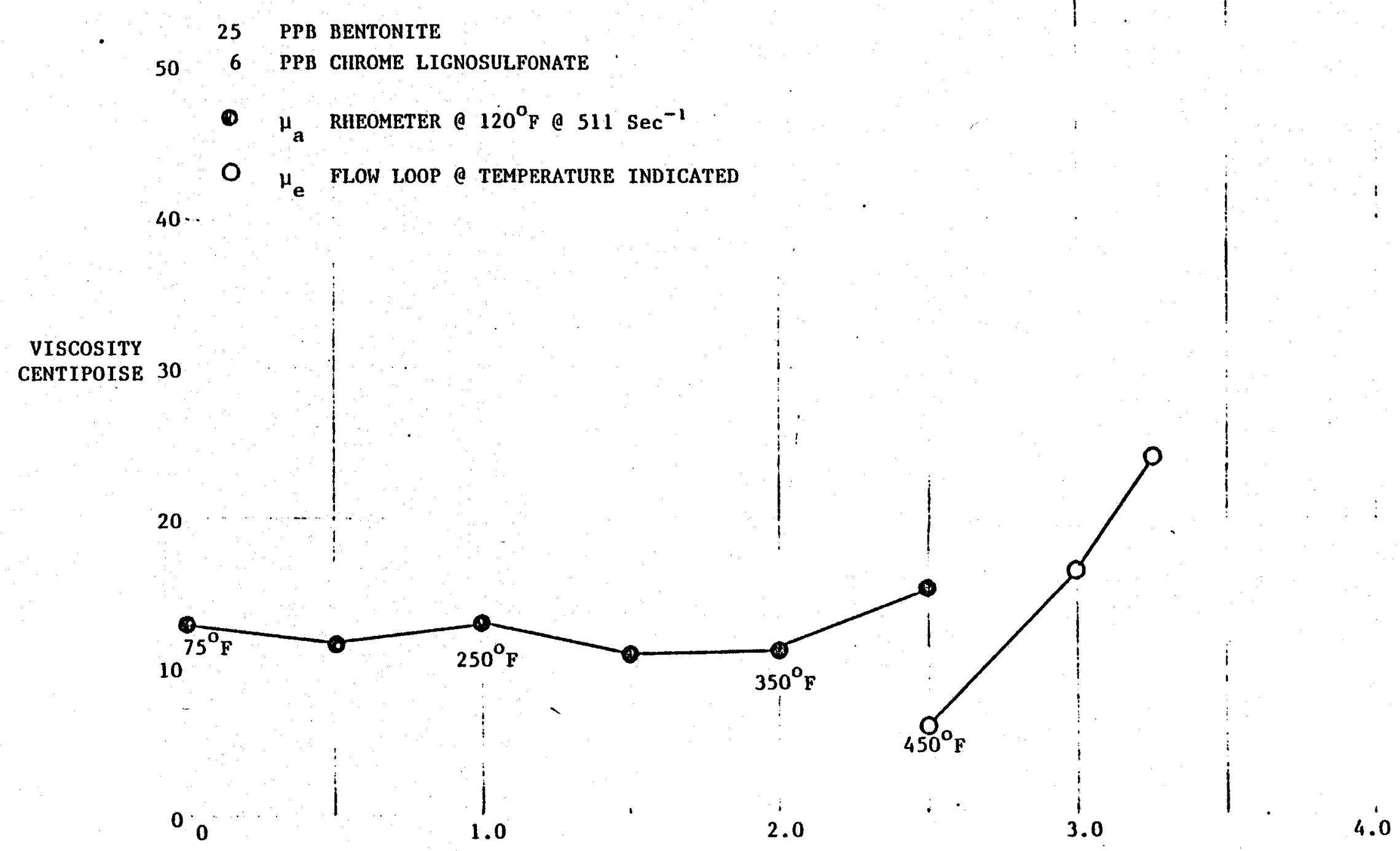

TIME HOURS 


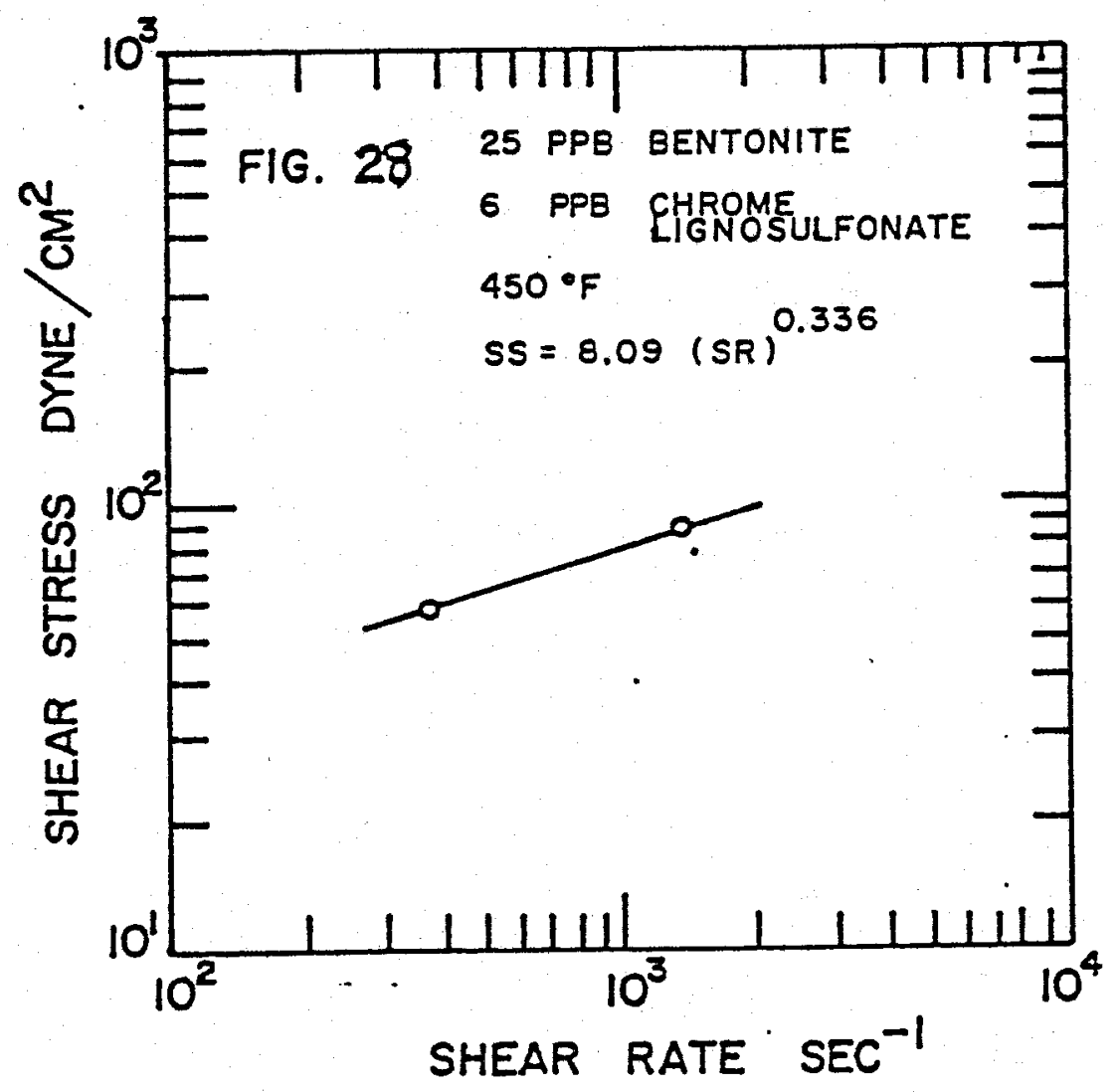


TABLE 16

BAROID RHEOMETER DATA
6 PPB CHROME LIGNOSULFONATE
e $120^{\circ} \mathrm{F} \mathrm{SR} \simeq 511 \mathrm{Sec}^{-1}$

6 PPB MODIFIED LIGNITE A

\begin{tabular}{|c|c|c|c|c|c|}
\hline $\begin{array}{c}\text { Af ter Temperature } \\
\text { Exposure } \\
\text { ( }{ }^{\circ} \text { Fahrenhe1t) }\end{array}$ & $\begin{array}{c}\mu_{a} \\
\text { Apparent } \\
\text { Viscosity } \\
\text { (Centipolse) }\end{array}$ & $\begin{array}{c}\text { PV } \\
\text { Plastic } \\
\text { Viscosity } \\
\text { (Centipoise) }\end{array}$ & $\begin{array}{c}\text { YP } \\
\text { Yield } \\
\text { Point } \\
(1 \mathrm{lb} / 100 \text { sq.ft.) }\end{array}$ & $\begin{array}{c}\text { Inftial Gel } \\
(1 \mathrm{~b} / 100 \mathrm{sq} . \mathrm{ft} .)\end{array}$ & $\begin{array}{c}10 \text { Minute } \\
\text { Gel } \\
(1 b / 100 \text { sq.ft.) }\end{array}$ \\
\hline 75 & 42.5 & 26 & 16.5 & 4.5 & 14 \\
\hline 250 & 38.5 & 30 & 18.5 & 3 & 3 \\
\hline 350 & 35 & 29.5 & 5.5 & 3.5 & 3.5 \\
\hline 450 & 27 & 20.5 & 6.5 & 3 & 3 \\
\hline
\end{tabular}


TABLE 17

1/4" TUBE VISCOMETER DATA
6 PPB CHROME LIGNOSULFONATE
$S R \simeq 760 \mathrm{Sec}^{-1}$

6 PPB MODIFIED LIGNITE A

\begin{tabular}{|c|c|c|c|c|c|c|}
\hline $\begin{array}{l}\text { Temperature } \\
\text { (OFahrenheit) }\end{array}$ & $\mathbf{p H}$ & $\underset{(\text { Psi) }}{\Delta \mathrm{P}}$ & $\begin{array}{l}\text { Shear Stress } \\
\left(\text { dyne } / \mathrm{cm}^{2}\right)\end{array}$ & $\begin{array}{c}\mu_{\mathrm{e}} \text { Effective } \\
\text { Viscosity } \\
\text { (Centipo1se) }\end{array}$ & $\begin{array}{c}\text { Power Law } \\
n\end{array}$ & $\underset{k}{\text { Coefficlents }}$ \\
\hline 75 & 9.6 & 5.4 & 428 & 56 & 0.761 & 2.59 \\
\hline 250 & 9.8 & 1.5 & 118 & 15 & 0.143 & 40.08 \\
\hline 350 & 9.1 & 0.8 & 60 & 8 & 0.172 & 16.73 \\
\hline 450 & - & 4.0 & 317 & 42 & 0.734 & 2.28 \\
\hline
\end{tabular}




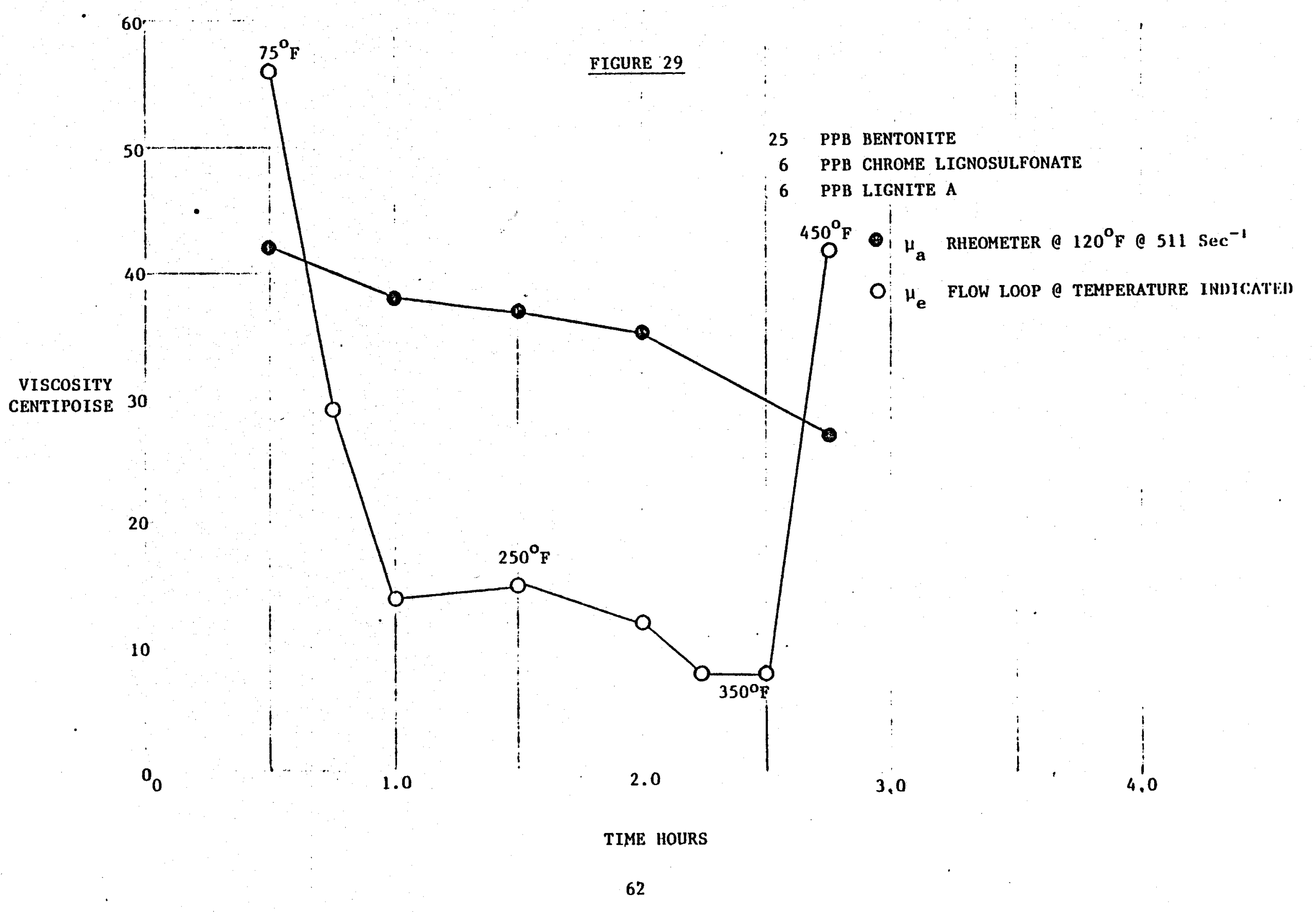



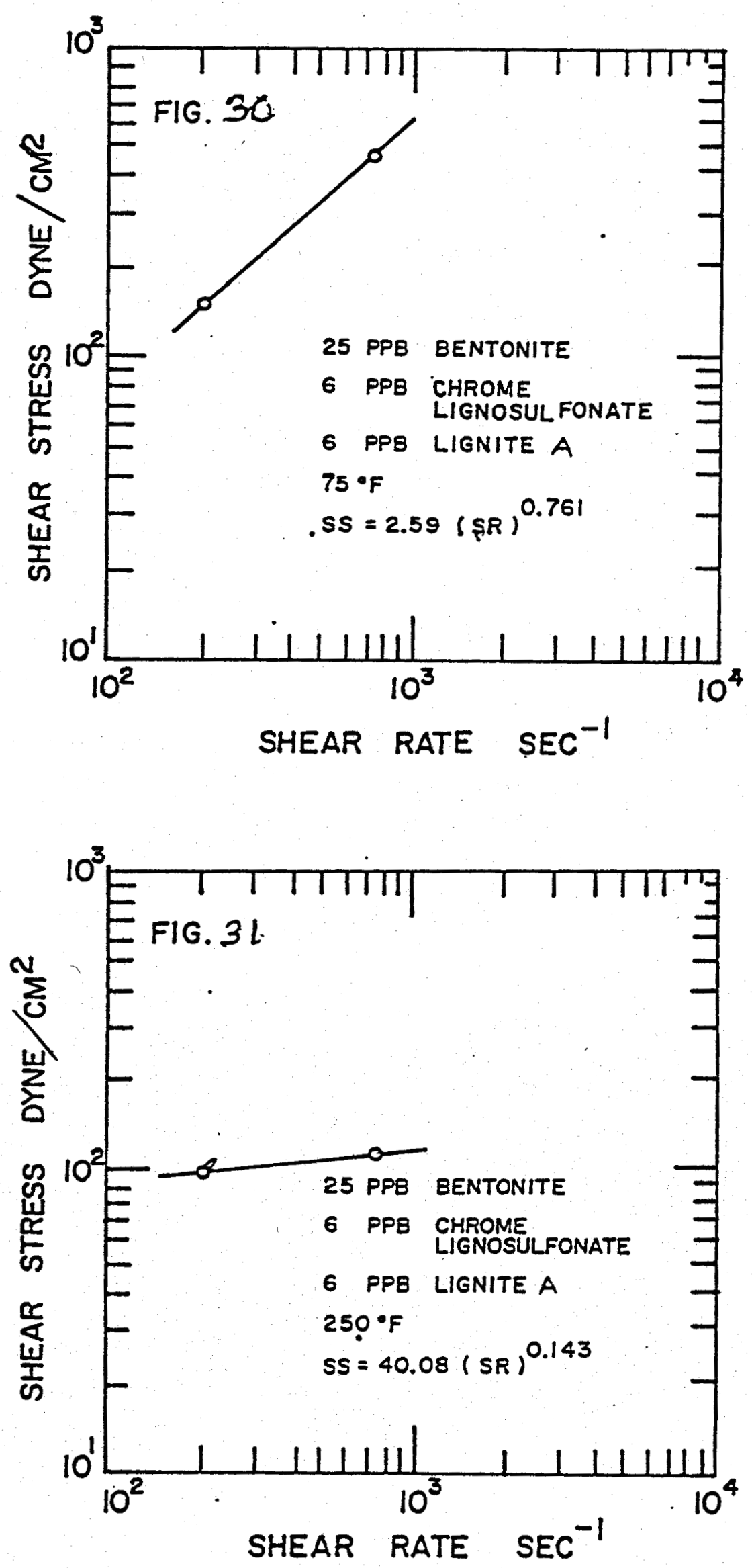

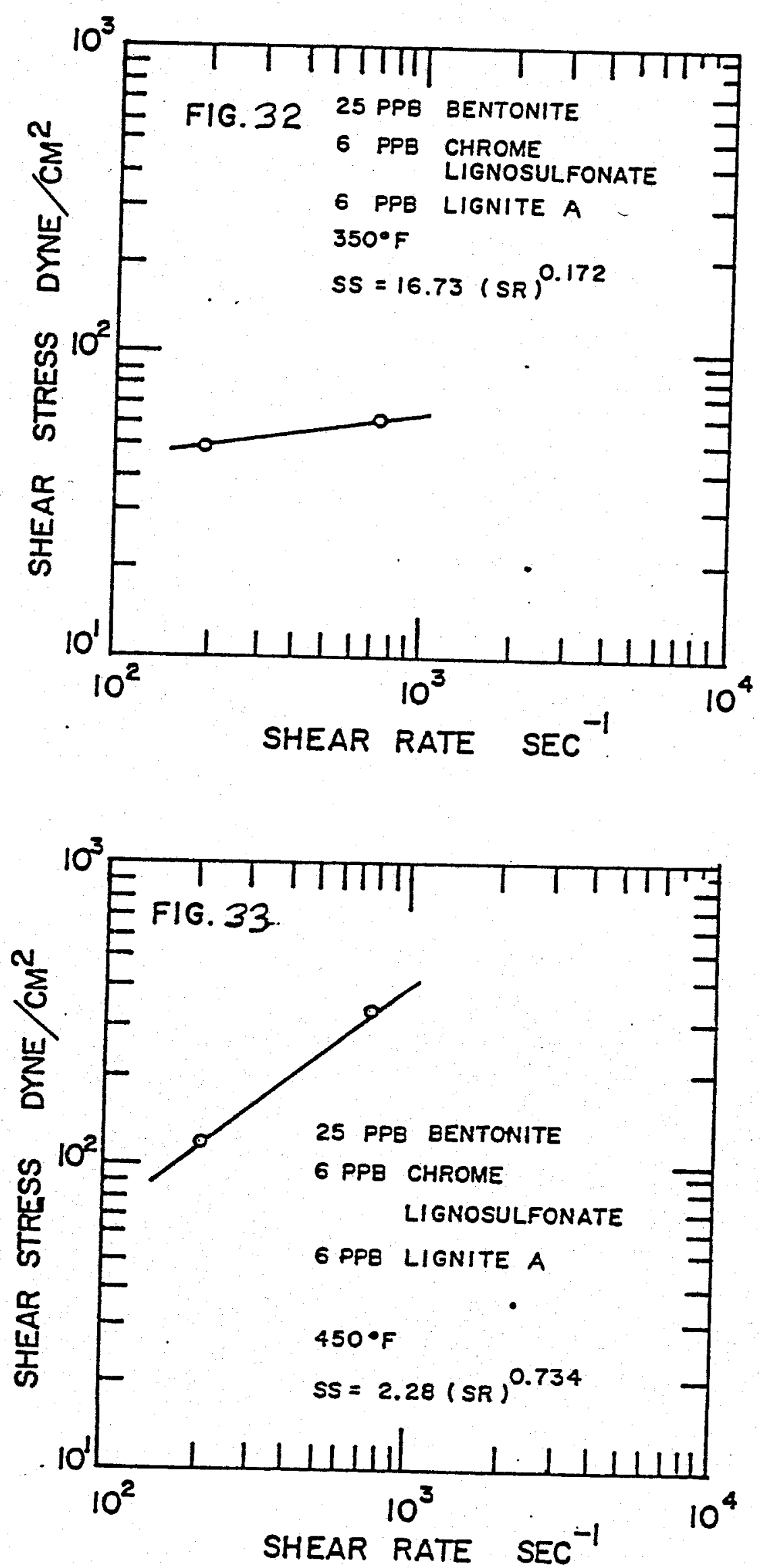


\section{TABLE 18}

BAROID RHEOMETER DATA

6 PPB CHROME LIGNOSULFONATE

(c) $120^{\circ} \mathrm{F}$ SR $\simeq 511 \mathrm{sec}^{-1}$

6 PPB MODIFIED LIGNITE B

\begin{tabular}{|c|c|c|c|c|c|}
\hline $\begin{array}{c}\text { After Temperature } \\
\text { Exposure } \\
\text { ( Fahrenheit) }\end{array}$ & $\begin{array}{c}\text { Apparent } \\
\text { Viscosity } \\
\text { (Centipoise) }\end{array}$ & $\begin{array}{c}\text { Plastic } \\
\text { Viscosity } \\
\text { (Centipoise) }\end{array}$ & $\begin{array}{c}\text { Yield } \\
\text { Point } \\
(1 b / 100 \text { sq.ft.) }\end{array}$ & $\begin{array}{c}\text { Inttial Gel } \\
(1 b / 100 \text { sq.ft.) }\end{array}$ & $\begin{array}{c}10 \text { Minute } \\
\text { Gel } \\
(1 \mathrm{~b} / 100 \mathrm{sq} . \mathrm{ft} .)\end{array}$ \\
\hline 75 & 12.5 & 10 & 2.5 & 1.0 & 1.0 \\
\hline 250 & 13.0 & 8.5 & 4.5 & 1.0 & 2.5 \\
\hline 350 & 8.0 & 5.5 & 2.5 & 1.0 & 2.5 \\
\hline
\end{tabular}


TABLE 19

$1 / 4$ " TUBE VISCOMETER DATA

6 PPB CHROME LIGNOSULFONATE

$S R \simeq 760 \mathrm{Sec}^{-1}$

6 PPB MODIFIED LIGNITE B

\begin{tabular}{|c|c|c|c|c|c|c|}
\hline $\begin{array}{l}\text { Temperature } \\
\text { ('oahrenhe1t) }\end{array}$ & pH & $\underset{(\mathrm{Ps} 1)}{\Delta \mathrm{P}}$ & $\begin{array}{l}\text { Shear Stress } \\
\left(\text { dyne } / \mathrm{cm}^{2}\right)\end{array}$ & $\begin{array}{c}\mu_{e} \text { Effective } \\
\text { Viscosity } \\
\text { (Centipoise) }\end{array}$ & $\begin{array}{c}\text { Power Law } \\
n\end{array}$ & $\begin{array}{c}\text { Coefficients } \\
k\end{array}$ \\
\hline 250 & 9.8 & 0.64 & 50.7 & 6.5 & 0.575 & 1.113 \\
\hline 350 & 7.5 & -3.1 & 245.0 & 32.0 & 0.083 & 126.540 \\
\hline
\end{tabular}




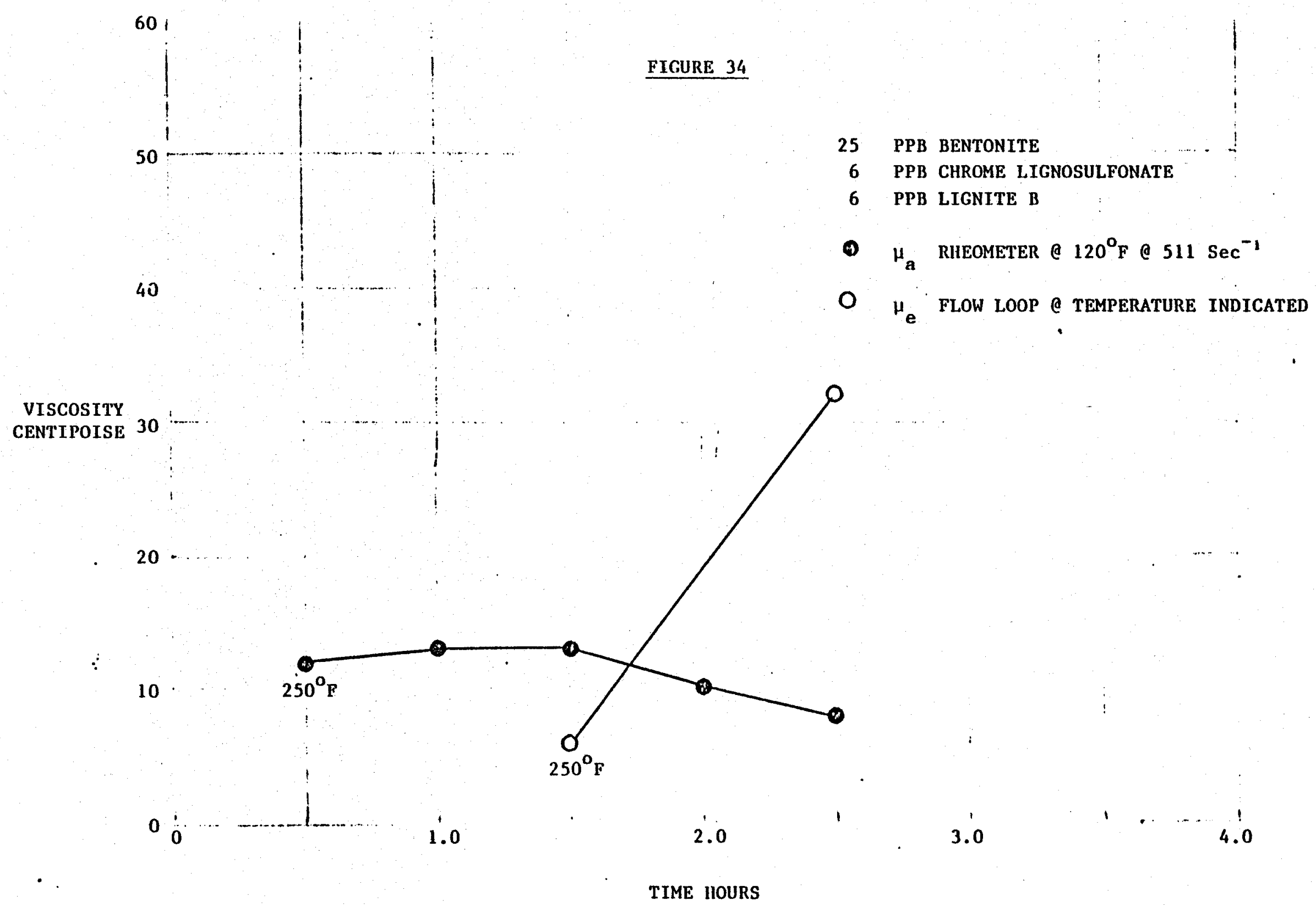



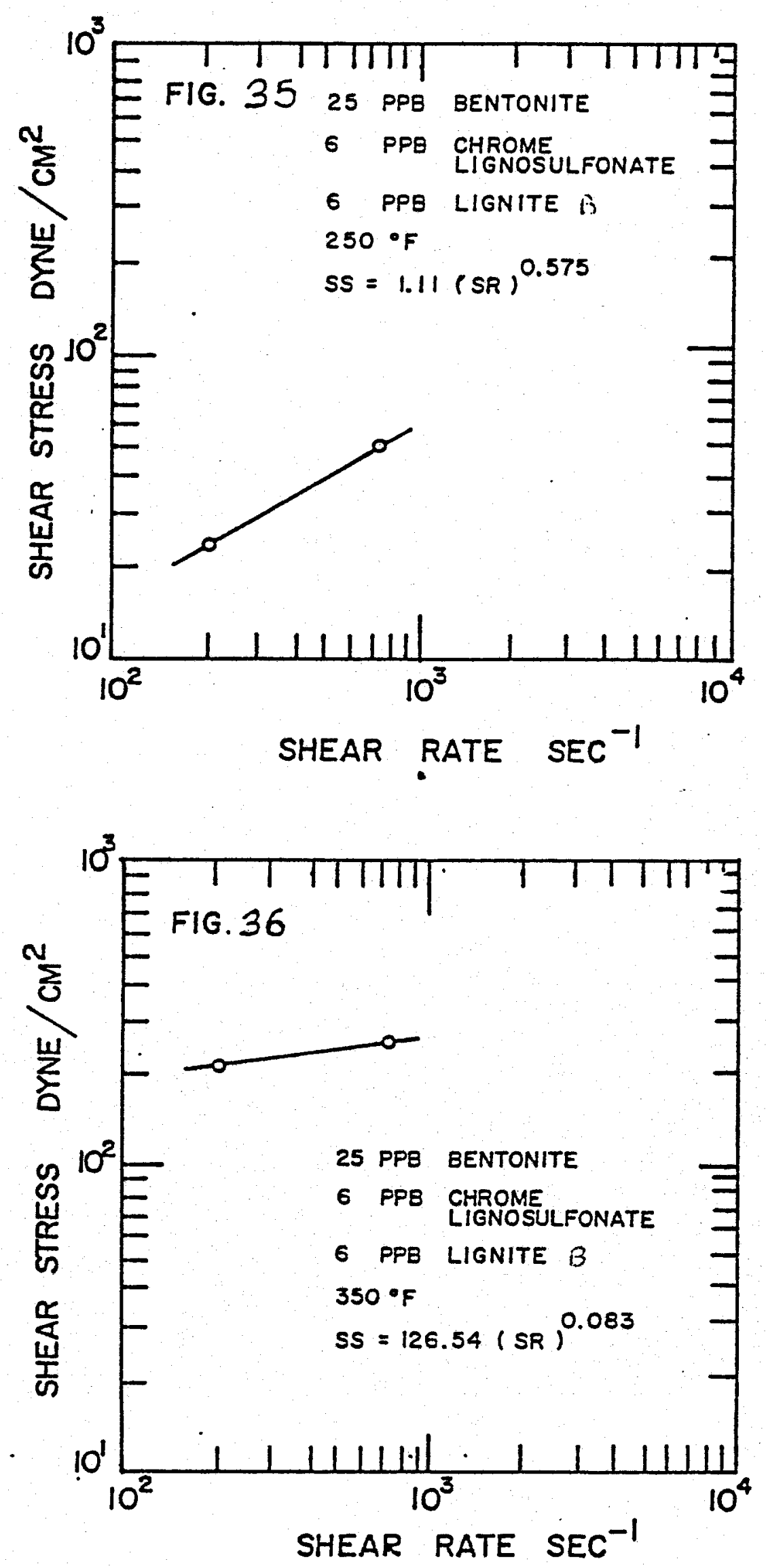


\section{MUD VISCOSITY DATA TO $400^{\circ} \mathrm{F}$ USING MAGCOBAR FLOW LOOP}

In order to obtain experience with flow loops and some early data it was decided to use the $400^{\circ}$ flow loop owned by Magcobar (Houston). These tests were carried out while the 0.U. flow loop was being built.

\section{HIGH TEMPERATURE DATA}

The viscosity of aine muds were measured at $200-400^{\circ} \mathrm{F}$ using a modified Magcobar flow loop and at $115^{\circ} \mathrm{F}$ using the Fann rheometer. The data shown in Figures 36 through 44 and Tables 20 through 28 suggest that (a) Lignosulfonates experience thickening at about $325^{\circ} \mathrm{F}$, (b) Polyanionic cellulose and modified polysaccaride become thickened at about $275^{\circ} \mathrm{F}$, (c) Starch experiences a gradual thickening with temperature, (d) HEC, Guar gum, and Xanthum gum experience a gradual thinning with temperature increases, and (e) CMC becomes thickened until $250^{\circ} \mathrm{F}$ is reached, then thins back with temperature increases. Although 29 flow loop tests were run, only 9 tests produced adequate data. Problems due to tube plugging and pump fallures made the other 20 sets of test data questionable.

\section{LOW TEMPERATURE DATA}

The $115^{\circ} \mathrm{F}$ apparent viscosity measured on the mud as it returned from the flow loop displayed the same viscosity-temperature trends as did 
the flow loop for the chrome Iignosulfonates, polyanionic cellulose, HEC, starch, modified polysaccaride, guar gum, and xanthum gum, but opposite effects were observed for CMC and ferrochrome lignosulfonates. The attached data summary sheets describe the muds tested and test observations.

EQUIPMENT USED

Figure 35 is a diagram of the modifled Magcobar flow loop. Modifications consisted of installing pressure taps across a 10 inch section of the high temperature part of the flow loop. Effective viscosities $\left(\mu_{e}\right)$, were calculated using the pressure drop across this section, $\Delta P$ in $p s i$, the flow rate through the section, $Q$ in $\mathrm{ml} / \mathrm{sec}$, and the equation,

$$
\mu_{e}=270 \frac{\Delta P}{Q}, c p
$$

at average shear rate $8 \mathrm{~V} / \mathrm{D}$ where,

$$
\frac{8 V}{D}=\frac{D \Delta P}{4 \mu_{e} L}
$$

After the mud was heated and tested at a given temperature it was cooled back to $115^{\circ} \mathrm{F}$ and its apparent viscosity $\left(\mu_{a}\right)$ measured using the Fann rheometer and the equation,

$$
\mu_{\mathrm{a}}=\mathrm{PV}+5.42 \frac{\mathrm{YP}}{\mathrm{Q}}, \mathrm{CP}
$$

This equation allows the calculation of the mud viscosity at approximately the same shear rate as the mud experienced in the flow loop test section. 
TEST No. 1

CONDITIONS OF TEST:

Base Mud Dionized Water +25 PPB Bentonite

Test Chemical Added, Quantity 6 PPB Ferrochrome Lignosulfonate

Shear Rate Range, $\mathrm{Sec}^{-1}$ 1,035 to 1,302

OBSERVATIONS :

When the test temperature reached $325^{\circ} \mathrm{F}$ the viscosity started increasing rapidly, as shown in Test No. 1, the Fann rheometer data did not display the thickening above $325^{\circ} \mathrm{F}$. 


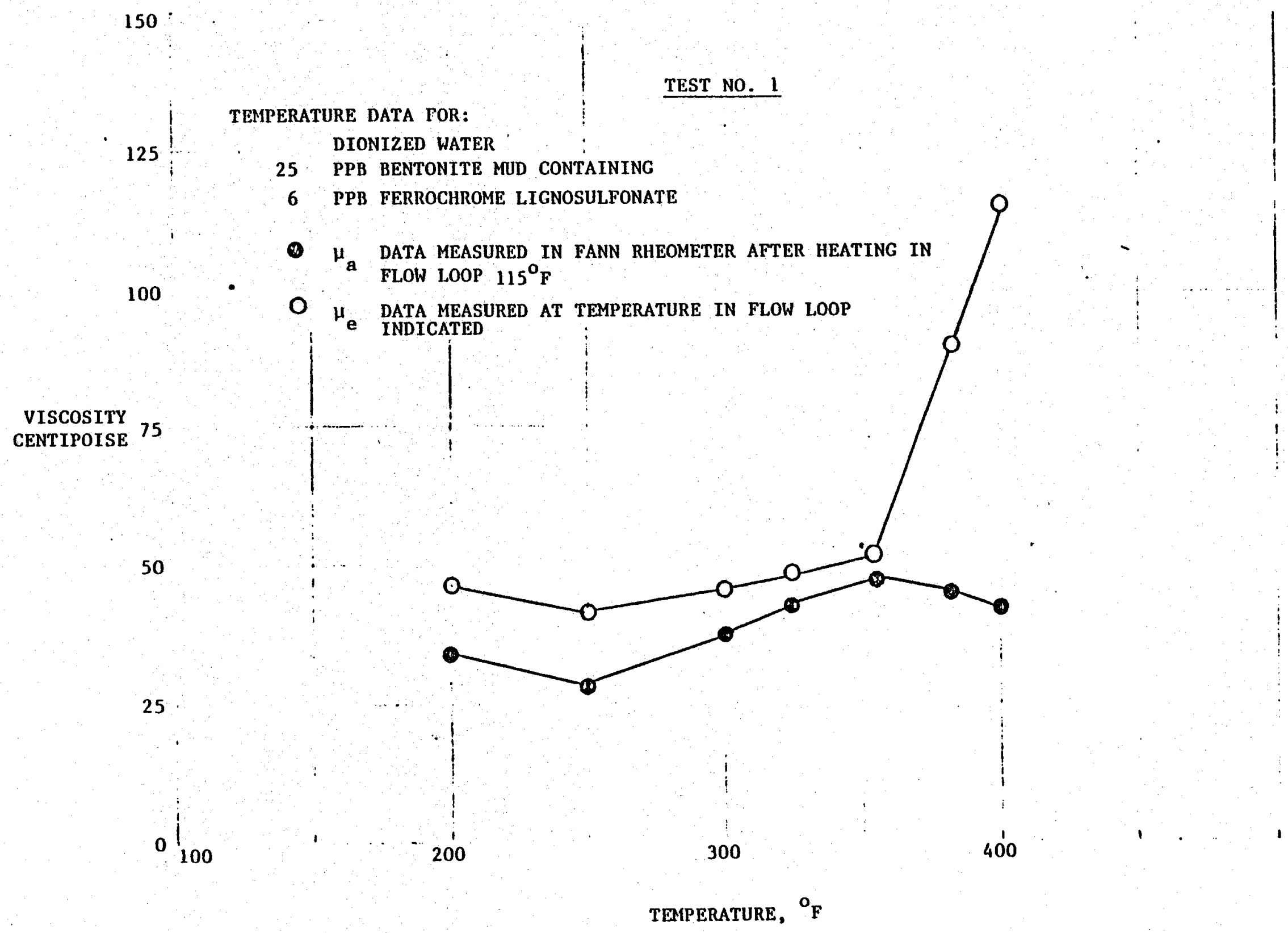


DATA SUMMARY SHEET

TEST NO. 2

CONDITIONS OF TEST:

Base Mud

Dionized Water +25 PPB Bentonite

Test Chemical Added, Quantity

6 PPB Chrome Lignosulfonate

Shear Rate Range, $\mathrm{Sec}^{-1}$

1,017 to 1,348

OBSERVATIONS :

As with ferrochrome 1ignosulfonate the viscosity started increasing when the temperature reached $325^{\circ} \mathrm{F}$, however the mud did not have as excessive thickening at $375^{\circ} \mathrm{F}$. 


\section{TEST NO. 2}

125 TEMPERATURE DATA FOR:

DIONIZED WATER

25 PPB BENTONITE

6 PPB CHROME LIGNOSULFONATE

$100 \quad \mu_{a}$ DATA MEASURED. IN FANN RHEOMETER AFTER HEATING IN FLOW LOOP $115^{\circ} \mathrm{F}$

O $\mu_{\mathrm{e}}$ DATA MEASURED AT TEMPERATURE IN FLOW LOOP INDICATED

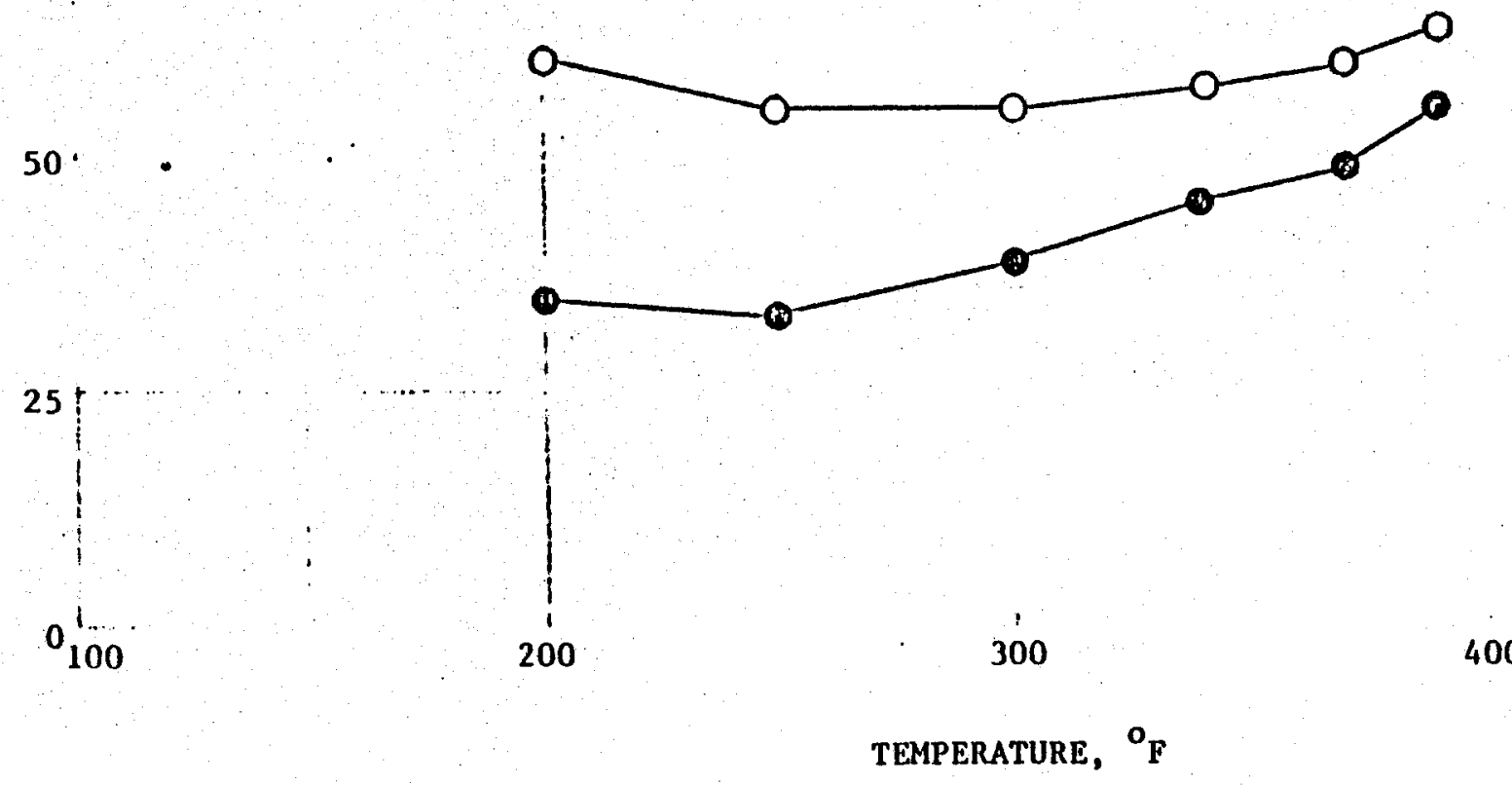


TEST No. 3

CONDITIONS OF TEST:

Base Mud

Dionized Water + 11.5 PPB NaCl + 25 PPB Bentonite

Test Chemical Added, Quantity

2 PPB Polyanionic Cellulose

Shear Rate Range, $\mathrm{Sec}^{-1}$ 781 to 1,905

OBSERVATIONS :

The flow loop and the Fann rheometer showed viscosity increases at $250^{\circ} \mathrm{F}$ and $275^{\circ} \mathrm{F}$ respectively. The flow loop shows a maximum viscosity of $55 \mathrm{CP}$ reached after $350^{\circ} \mathrm{F}$. 


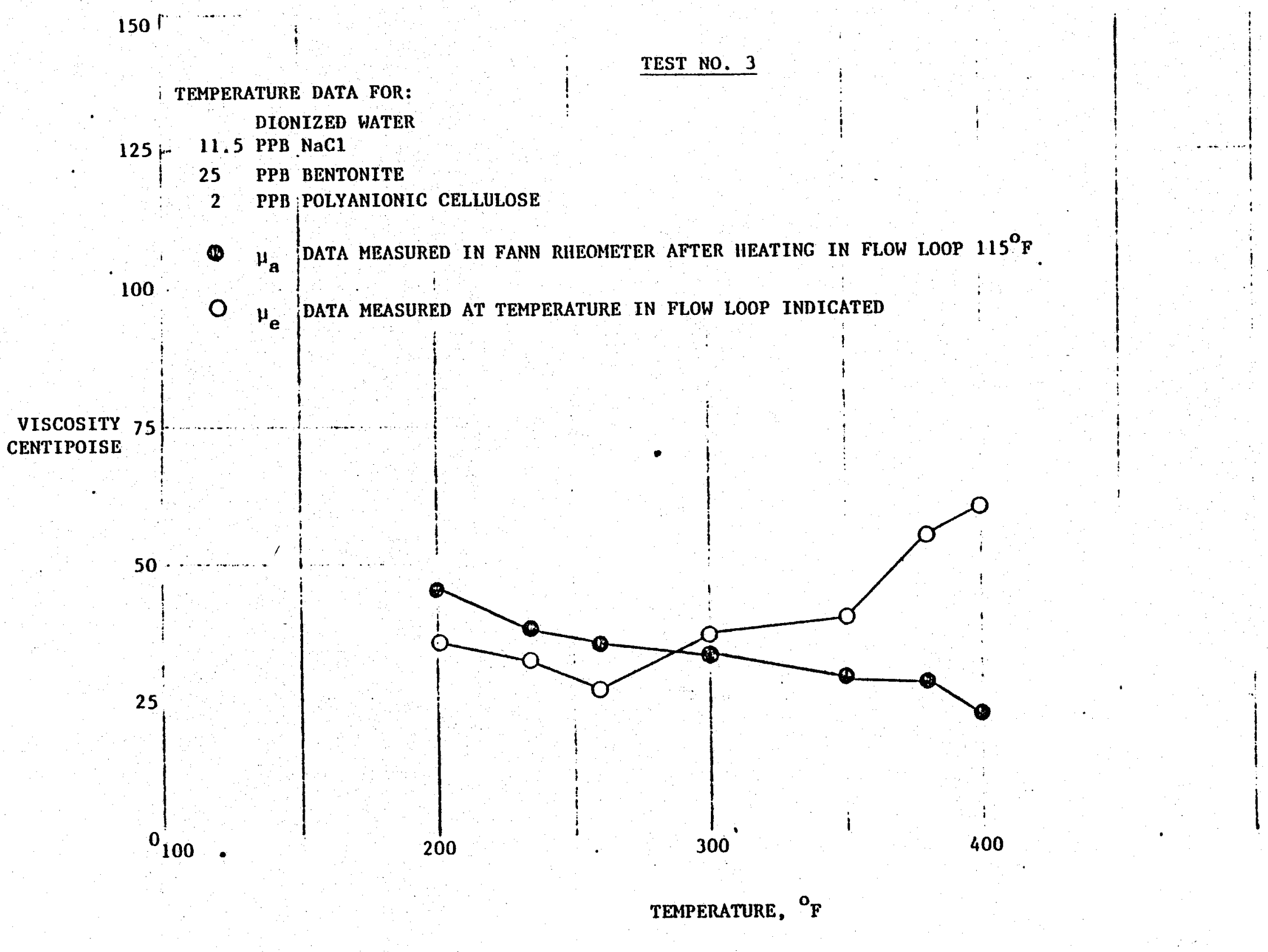


78

DATA SUMMARY SHEET

TEST NO. 4

CONDITIONS OF TEST:

Base Mud

Test Chemical Added, Quantity

Shear Rate Range, $\mathrm{Sec}^{-1}$
Dlonized Water + 11.5 PPB NaCl + 25 PPB Bentonite

6 P PB Modified Polysaccharide

896 to 1,558

OBSERVATIONS :

This chemical shows a viscosity reduction at $275^{\circ} \mathrm{F}$ with no excessive

thickening up to $375^{\circ} \mathrm{F}$. 

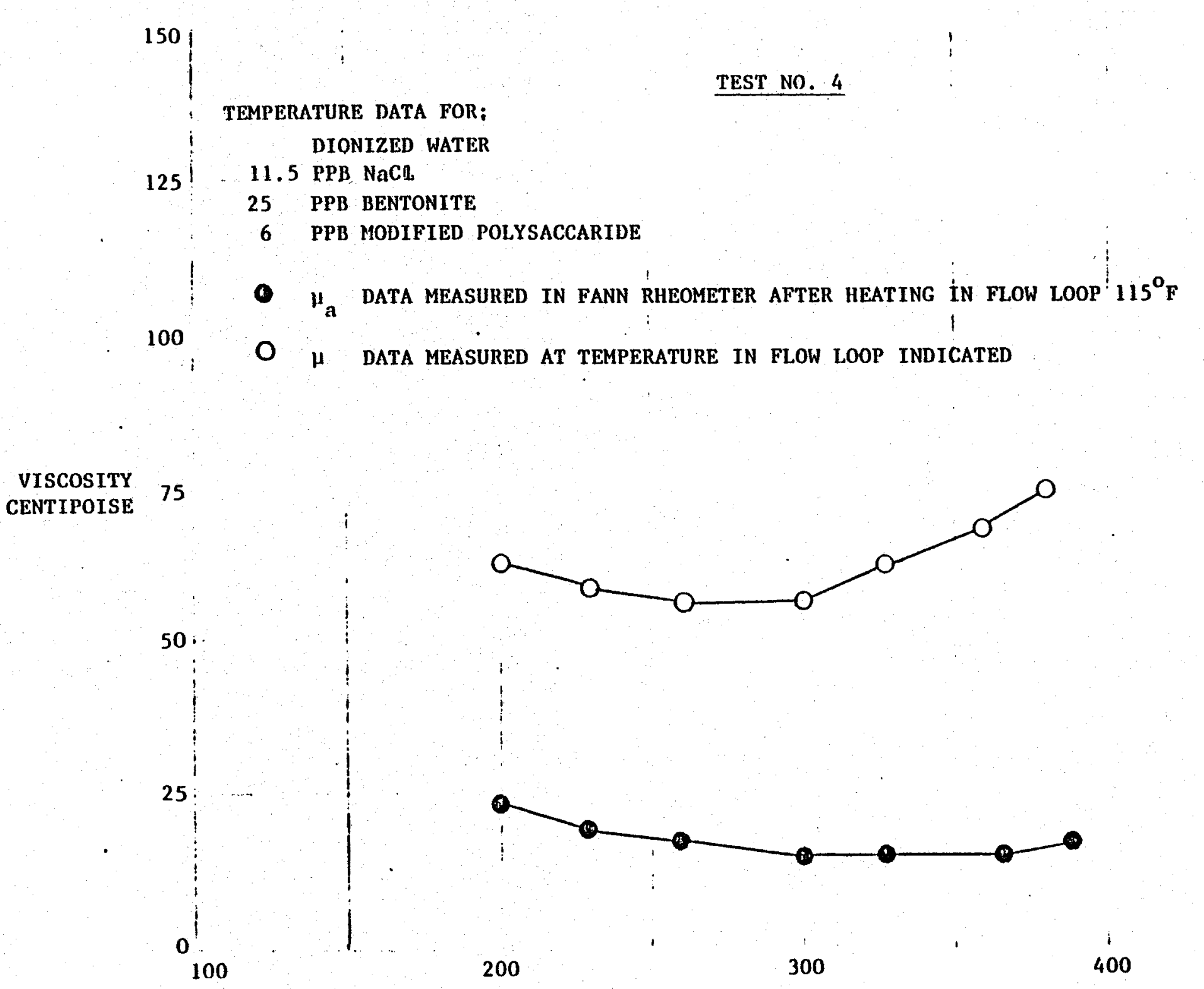

TEMPERATURE, ${ }^{\circ}$ F 
TEST NO. 5

CONDITIONS OF TEST:

Base Mud

Dionized Water + 11.5 PPB Salt + 25 PPB Bentonite

Test Chemical Added, Quantity

6 PPB Starch

Shear Rate Range, $\mathrm{Sec}^{-1}$

1,177 to 2,012

OBSERVATIONS :

As temperature increases, the viscosity of the system goes up

gradually. 
1501

TEMPERATURE DATA FOR:

TEST NO. 5

DIONIZED WATER

VISCOSITY

CENTIPOISE 75 .

11.5 PPB SALT

25 PPB BENTONITE

6 PPB STARCH

(1) $\mu_{a}$ DATA MEASURED IN FANN RHEOMETER AFTER HEATING IN FLOW LOOP $115^{\circ} \mathrm{F}$

$100-0 \quad \mu_{e}$ DATA MEASURED AT TEMPERATURE IN FLOW LOOP INDICATED

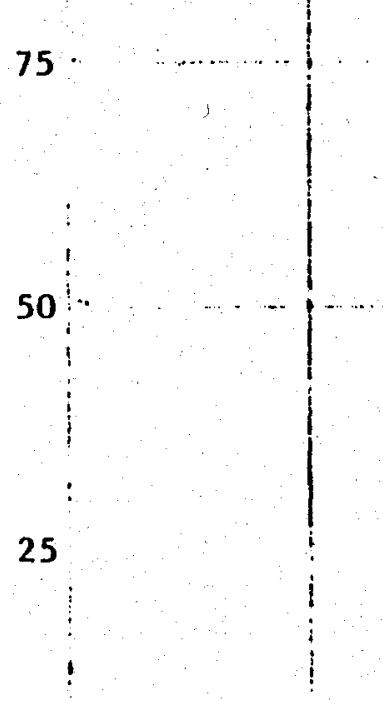

$0_{100}$

200

300

400

TEMPERATURE, ${ }^{o_{F}}$ 
82

DATA SUMMARY SHEET

TEST NO. 6

CONDITIONS OF TEST:

Base Mud

Test Chemical Added, Quantity

Shear Rate Range, $\mathrm{Sec}^{-1}$

OBSERVATIONS :
DIonized Water

2 P PB Hydroxyethyl Cellulose

Increase in temperature produces a reduction in viscosity in both

the flow tube data and the Fan n rheometer data. 


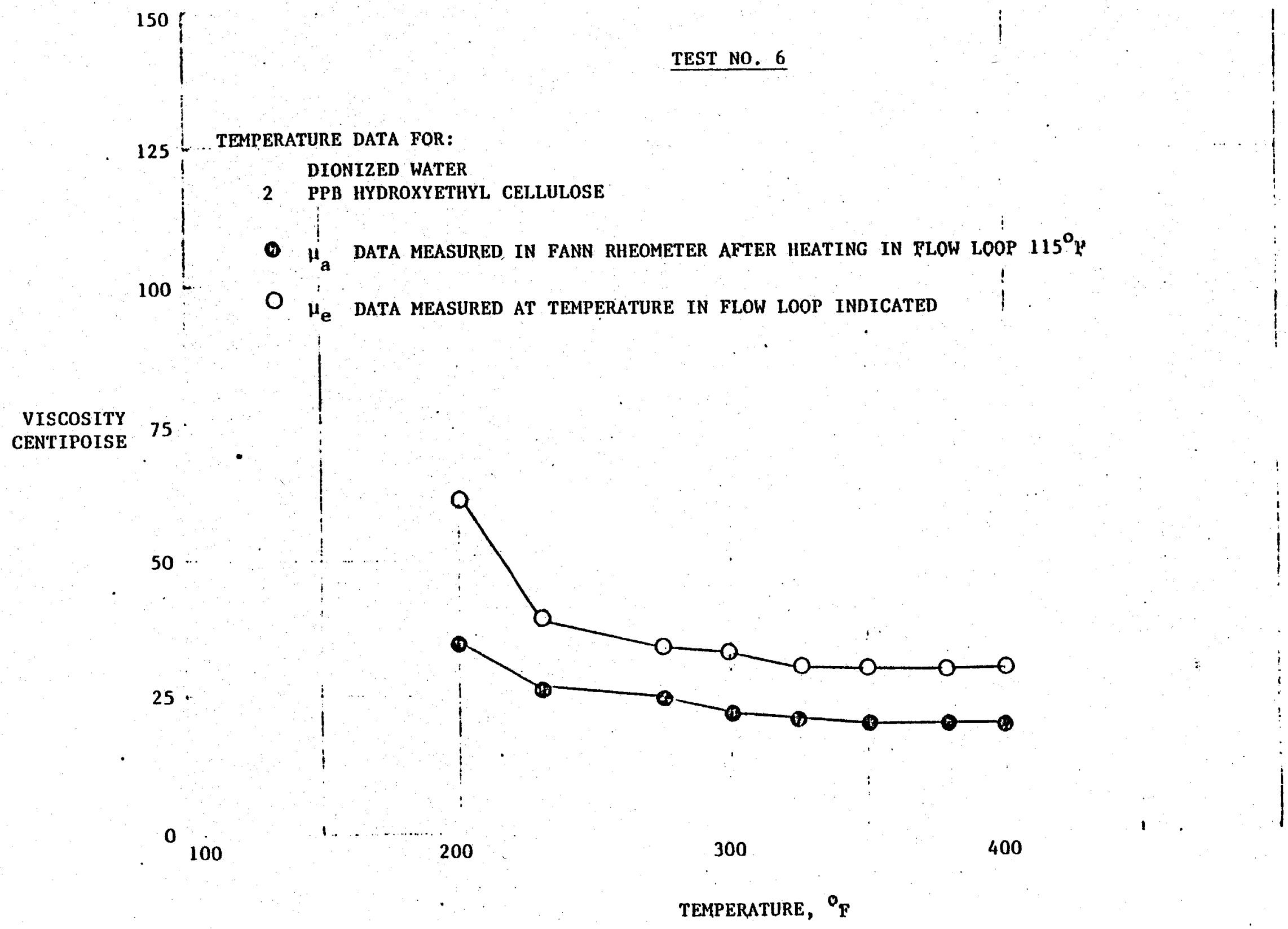


DATA SUMMARY SHEET

TEST NO. 7

CONDITIONS OF TEST:

Base Mud

Test Chemical Added, Quantity

Shear Rate Range, $\operatorname{Sec}^{-1}$.
Dionized Water

2.3 PPB Guar Gum

811 to 1,675

OBSERVATIONS :

As with HEC the viscosity of Guar gum decreases with temperature. 
$150 r$

$\therefore$

TEST NO. 7

TEMPERATURE DATA FOR: DIONIZED WATER

$125 \quad 2.3 \mathrm{PPB}$ GUAR GUM

- $\mu_{a}$ DATA MEASURED IN FANN RHEOMETER AFTER heATING IN FLOW LOOP $115^{\circ} \mathrm{F}$

$100 \quad 0 \mu$ DATA MEASURED AT TEMPERATURE IN FLOW LOOP INDICATED

VISCOS ITY

CENTIPOISE
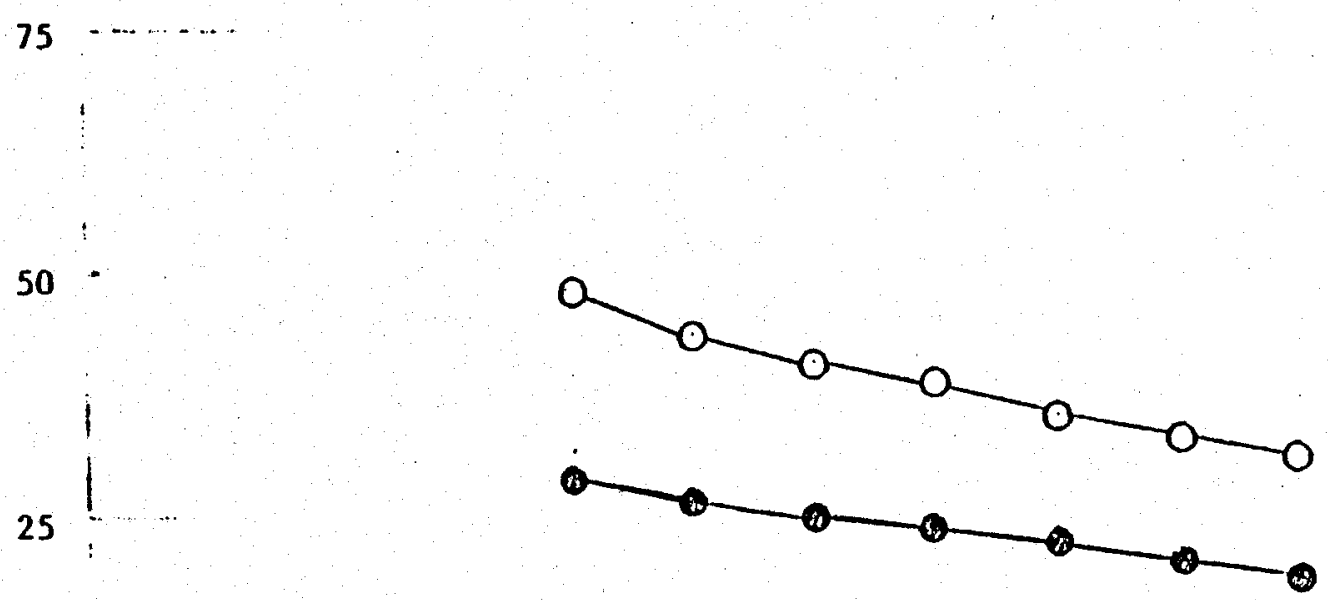

0100

200

300 
DATA SUMMARY SHEET

TEST NO. 8

CONDITONS OF TEST:

Base Mud

Dionized Water

Test Chemical Added, Quantity

2.3 PPB Xanthum Gum

Shear Rate Range, $\mathrm{Sec}^{-1}$

811 to 1,398

OBSERVATIONS :

The viscosity of Xanthum gum - water solution decreases with temp-

erature. At about $250^{\circ} \mathrm{F}$ the viscosity stabilizes. 

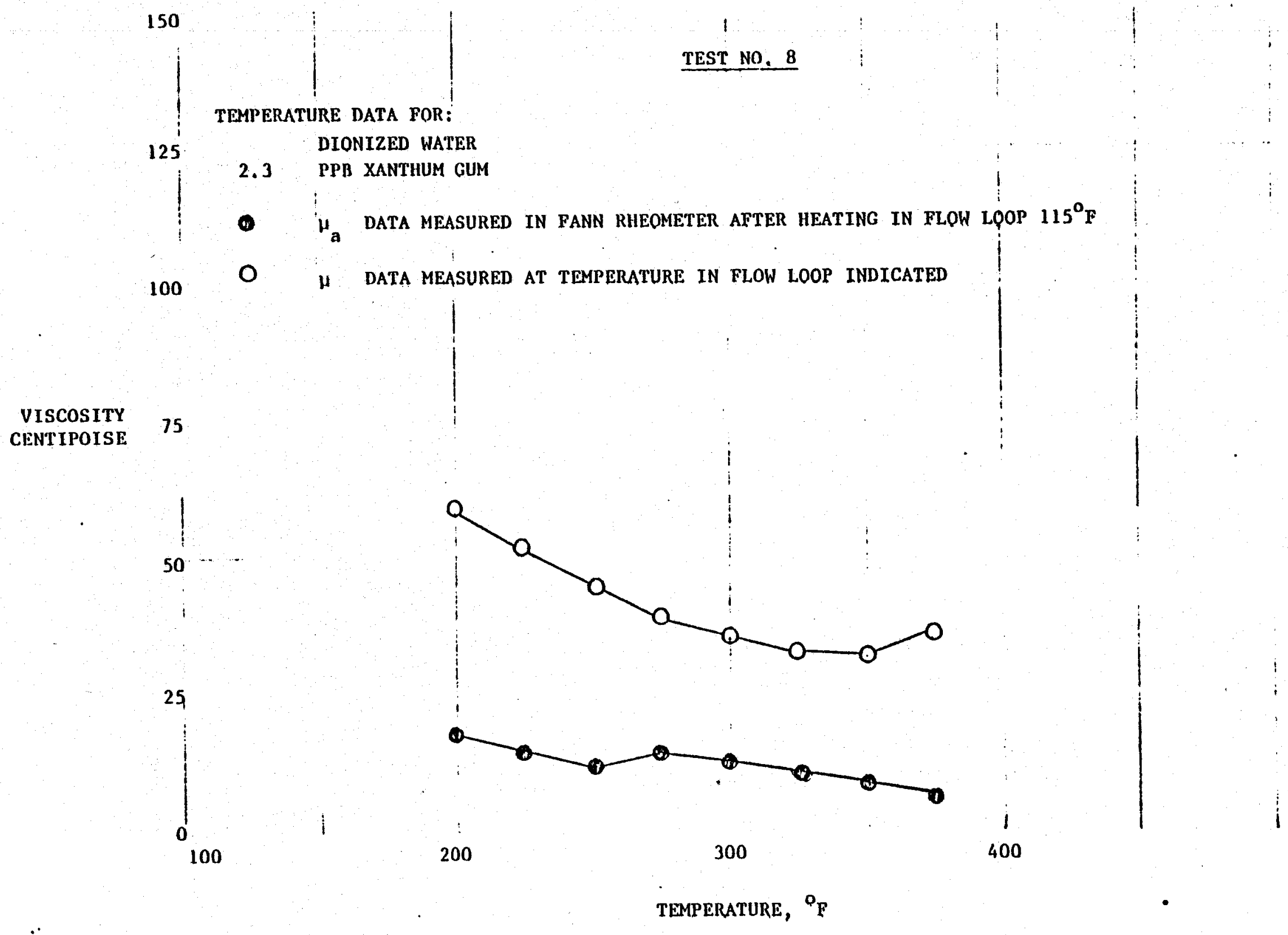


\section{DATA SUMMARY SHEET}

TEST NO. $\underline{9}$

CONDITIONS OF TEST:

Base Mud

Dionized Water + $11.5 \mathrm{PPB} \mathrm{NaCl}+$ 25 PPB Bentonite

Test Chemical Added, Quantity

2 PPB CMC

Shear Rate Range, $\mathrm{Sec}^{-1}$

235 to 1,605

OBSERVATIONS :

CMC in a salt water mud thickens until $250-275^{\circ} \mathrm{F}$ is reached, then

it thins back. The Fann rheometer data shows a very different response than the flow loop. 
150

\section{TEST NO. 9}

TEMPERATURE DATA FOR:

I 25 DIONIZED WATER

11.4 PPB NaCl

2 PPB CMC

100

$\mu_{a}$ DATA MEASURED IN FANN RHEOMETER AFTER HEATING IN FLOW LOOP $115^{\circ} \mathrm{F}$

$\mu_{e}$ DATA MEASURED AT TEMPERATURE IN FLOW LOOP INDICATED

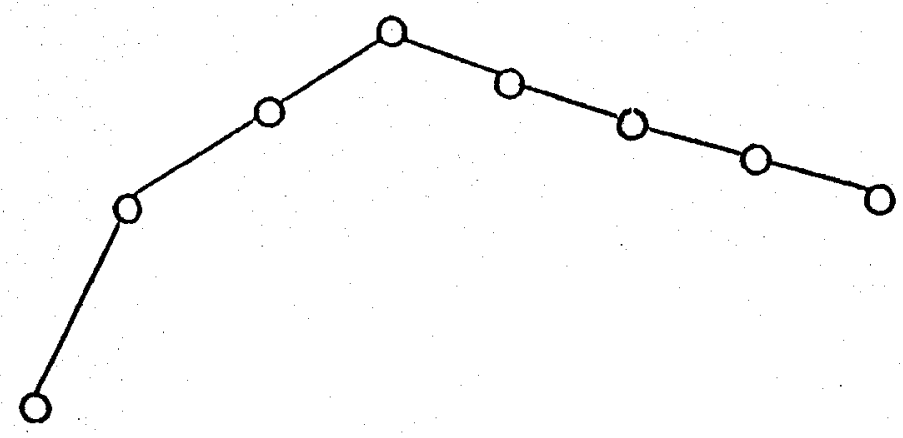

25

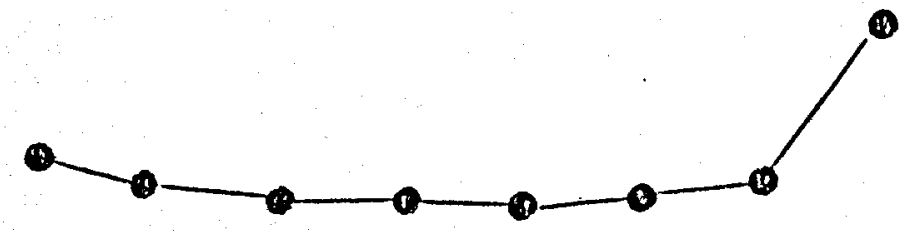

$0_{100}$

200

300

400

TEMPERATURE, ${ }^{\circ} \mathrm{F}$ 
APPENDIX A 
SYSTEM DESIGN FOR HIGH TEMPERATURE TESTING AND STABILIZATION

OF DRILLING FLUIDS

CHAPTER I

INTRODUCTION

The effects of high temperature on drilling fluid properties become very serious as deep wells or geothermal wells are drilled. Some drilling fluld compounds such as phosphates and tannin become thermally unstable and degrade at relatively low temperature, approximately $200^{\circ} \mathrm{F}$. Even the more temperature-resistant and widely used thinners such as chrome lignosulfonate can show instability after prolonged exposure to temperatures much beyond $300^{\circ} \mathrm{F}$. Generally, mud degradation indicates decomposition, increasing corrosivity, flocculation, which in turn results in filtrate loss, or a combination of these. It is important to realize at what temperatures mud chemicals start to loose their function and how serious the adverse effects are, in order to reduce drilling costs and to prevent the potential hazards of pressure surges. In the search for temperature-stable materials, much effort has been made to establish adequate laboratory procedures for determining thermal stabilitfes of drilling fluids although no standardized procedure or apparatus has been widely accepted. 
There are two general approaches to this problem: the aging cell method and circulation method. To age or stabilize the mud, the former requires time-consuming manipulation in the laboratory while the latter takes much shorter time and less effort to obtain properly aged muds. Other advantages of the latter method are the capabilities of continuous monitoring of mud properties and laboratory simulation of the muds produced during field drilling.

In this study a circulation system is designed for studying such mud properties as viscosity, filtration and corrosion up to $550^{\circ} \mathrm{F}$. The mud sample is pumped through a flow loop consisting of heating, test and cooling sections. The fluid is subject to intensive shearing by springloaded valves to promote mud aging and maintain a sufficient pressure to retard boiling. The cycle is repeated about once in two minutes depending on flow rate. The cool advancing mud is heated by the hot returning mud in a mud-to-mud. heat exchanger of paralleled tubes. Electric heater elements and in a molten salt heat exchanger. The hot mud is cooled by water which is flowing in the annulus of a double pipe heat exchanger. Pressure drops between taps along the lengths of three different sized tubes are measured in a test section to determine rheological parameters at elevated temperatures for non-Newtonian drilling fluids.

In a similar Magcobar system, only one pressure drop measurement is made to determine the effective viscosity of test fluids. Viscosities at two intermediate shear rates and one low shear rate are of concern in this system to obtain a more complete picture of rheological properties. By using strip recorders, temperature and pressure recordings are made in a continuous manner so that (1) the system temperatures can 
be well monitored and controlled, and (2) aging histories of different fluids can be compared directly and analyzed.

This Appendix will emphasize general design considerations and design calculations of heat transfer units and tube viscometer sections. Thermal properties of drilling fluids at elevated temperatures are estimated tentatively for use in heat transfer calculations. Brief discussions of fouling problems and application of non-Newtonian rheology to flocculation characteristics are also included. 
CHAPTER II

RELATED PROBLEMS

Because of the complex interrelated effects of temperature and aging time on mud properties it is necessary to distinguish the individual effects of:

1. temperature at which the test mud has been aged,

2. time taken to stabilize the test mud,

3. temperature at which the properties are measured after or during aging,

4. the manner the test mud has been aged, and

5. mud type, pH, concentration, electrolyte content and other mud characteristics.

Drilling fluids usually show fairly well defined temperatures at which abrupt increases of yield points, excessive fluid losses and abnormal corroston start to occur, but it is still difficult to define the maximum operating temperatures at which the fluids retain their usefulness. As for rheological properties, the low shear rate viscosity cannot be deduced from the knowledge of high shear rate viscosity at elevated temperatures. High shear rate viscosity may be only slightly affected by the soaring yield point caused by flocculation, while the low shear rate viscosity is controlled predominantly by it, (Chapter IV). 
Therefore, it seems necessary to refer the temperature limits of drilling fluids to particular shear rates. 


\section{CHAPTER III}

\section{PRELIMINARY DESIGN CONSIDERATIONS}

There are a wide variety of drilling fluids to be tested with this system. It is important to design a flexible and really capable system to meet the requirements of all types of test fluids with considerably different viscosities, mud weights and other characteristics. It is also possible that circulation rate or pressure may be varied in the experiments. However, the maximum achievable temperature of the system, or the maximum heat transfer rates of the heating and cooling sections, depends on the mud properties. For example, the viscous energy dissipation of seriously flocculated muds could significantly affect the heat transfer characteristics. The variation of viscosity with temperature influences convective heat transfer, especially for the units with high heat flux densities such as the electrical heating section. In other words, the system capacity depends on fluld properties themselves which are unfortunately unknown in the designing stage.

A computer program was written to read in all possible combinatlons of mud properties, and to calculate the desired sizes of heat transfer units for each combination, as will be discussed in Chapter VII. The calculations result in a range of slzes for the desired system capacity. The largest reasonable value in the range is considered as the desired answer. 
Another problem with drilling fluids is its fouling and probable corrosive characteristics. Special attention is needed in the heat exchanger design:

1. Test fluids should be in the "tube" side of heat exchangers, not the shell side.

2. For maintenance convenfence, mud tubes should be relatively straight.

3. Provision should be made to keep the temperature distributions around the mud tubes as even as possible, due to the high heat flux densities generated by electrical heater elements connected to the tubes. 
CHAPTER IV

\section{BEHAVIOR OF DRILLING FLUIDS AT ELEVATED TEMPERATURES}

The rheological and filtration properties of drilling fluids are closely assoclated with flocculation. Flocculation at elevated temperatures, therefore, make viscosity and filtration the most prominent parameters to be affected by temperature. Viscosity usually shows a marked variation with temperature. Yield point, may also vary erratically before reaching a critical temperature. Beyond this point, mud particles react violently and the yield point can rise considerably.

Figure 47 shows some examples of temperature dependent driling fluids in terms of flow behavior index $n$. The diversity of the parameter introduces difficulties in the sizing of heat transfer units.

Apparent viscosities at high and low shear rates reveal very different characteristics with varying temperature. Low shear rate viscosity will reflect the abrupt increase of yield point approaching the flocculation temperature. The higher the shear rate, the less the flocculation effect on the apparent viscosity.

At very high shear rates power law fluids exhibit only minor changes in apparent viscosity. Under these limited conditions an equiv- . alent PV (plastic viscosity) and YP (yield point) can be defined for the power law fluid. A power law fluid by definition does not have any yield point, consequently this calculated YP is only a convenience. 
FIGURE 38

\title{
SOME EXAYPLES OF TEMERATURE DEPENDEXCE OF DRIIIING FLUIDS IN
}

\author{
TERMS OF FLOW BEHAVIOR INDEX
}

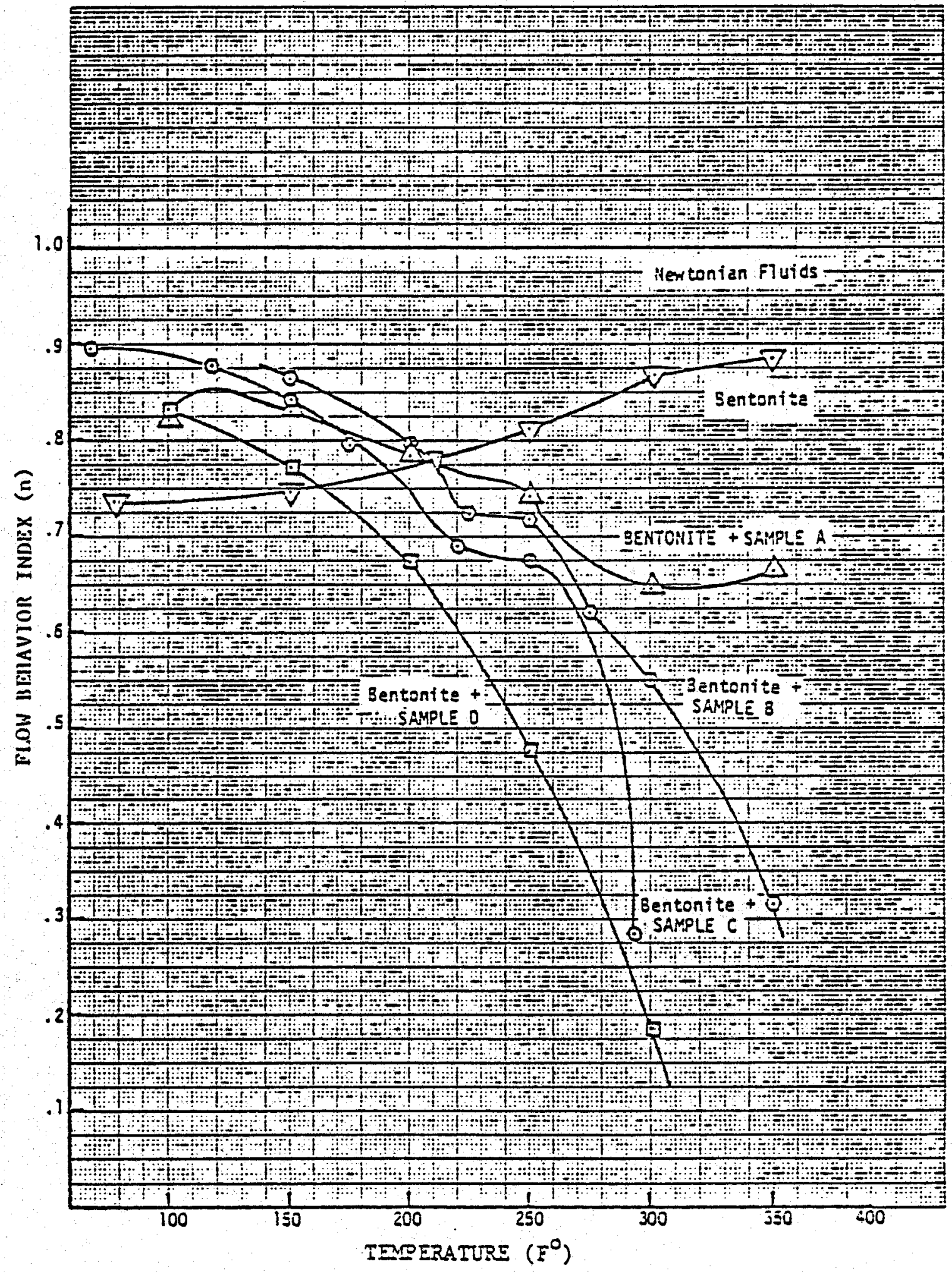


The advantages of using $n$ from the power law model,

$$
\tau_{y x}=K\left(\frac{d u}{d y}\right)^{n}
$$

are:

1. It is dimensionless and can be compared directly among samples,

2. It's magnitude indicates the relative influence of the hIgh and low shear rate viscosities, since apparent viscosity for a power law fluid is

$$
\mu_{a}=K\left(\frac{d u}{d y}\right)^{n-1}
$$

3: It will reveal the extent of departure from Newtonian characteristics.

As for the temperature effects on filtration properties of drilling fluids, the API fluid losses usually show increases after aging at elevated temperatures. Dynamic filtration also reveals a similar trend, at least in the tenperature ranges in which previous studies have been done. Theoretically, dynamic fluid loss is approximately proportional to the shear stress at the surface of a filter cake and in inverse proportion to the filtrate viscosity. As temperature increases, filtrate viscosity will usually show a decrease and this tends to Increase the filtrate loss. On the other hand, shear stresses may increase or decrease with temperature and shear rate. In conclusion, dynamic filtration tends to increase with temperature in the majority of cases, although theoretically there are some possibilities of the reversed trend. 
Serious corrosion problems arise with increased temperatures. While deteriorating at elevated temperatures, lignosulfonates can release hydrogen sulfide and cause steel embrittlement. 


\section{CAAPTER V}

\section{SELECTION OF HEAT TRANSFER UNITS}

There are wany alternatives to achieve the required temperature for the system. A cholce among steam, external electrical heating, internal electrical heating and heat transfer liquids were considered. The following shows advantages and disadvantages of each unit:

\section{Steam}

EIgh pressure superheated steam is available at temperature of $700^{\circ} \mathrm{F}$ and 400 psi pressure. Higin pressure stean is usually desirable in heat transfer operations if it is saturated. However, this steam has excessive superheat which requires extended surfaces such as longitudinal fins in a double pipe to improve heat transfer performance. The main problem with using steam at the University of Oklahoma was the high cost of installing piping and flashing system to reduce the steam to atmospheric pressure. It was therefore not adopted.

\section{External Electric Heating}

It is so called external because an electric element is silver soldered along the side of a mud tube and heat is provided from the outside, as compared to the next arrangement. Electric heating was first used by Magcobar to heat 
drilling mud samples, it has the following advantages:

a. It is highly efficient as far as the thermodynamical effectiveness is concerned. Almost all the heat energy generated in the resistance wire is absorbed by the flu1d, provided the system is insulated.

b. The control of heat flux and therefore, of the maximum systen cemperature is relatively simple.

c. Very high heat flux density is obtainable.

d. It's modular nature provides flexibility and makes further extension of the unit relatively easy.

\section{FIGURE 39}

POSSIBLE ARRAYGEMENT OF GEATER ELEEENT AND TUBING IN CROSS SECTION TO ENEANCE EEAT TRANSFER BY NATURAI CONVECTION
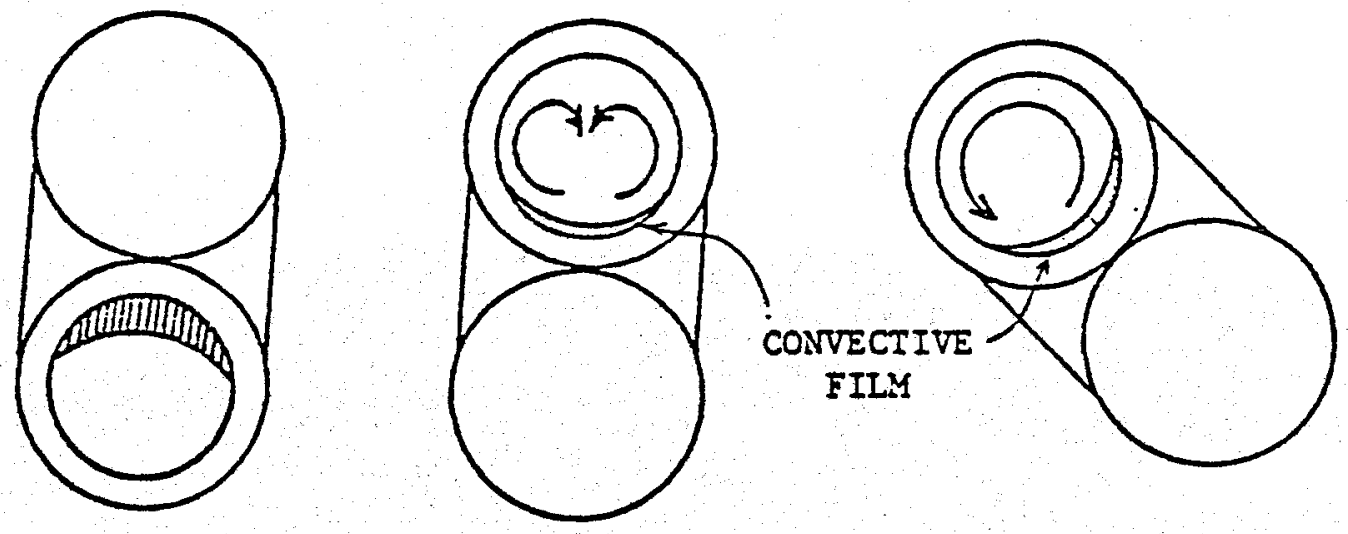

Eeater element above the tube

Poor in Convection

(a)
Heater element below the tube

Fair in Convection

(b) Eeater element
$45^{\circ}$ eilted

Good in Convection

(c) 
A drawback associated with external heating is the uneven temperature distribution around the tube periphery, due to excessive heat flux density near the tube wall adjacent to the heater element. It has caused serious fouling or 'plugging' in the tube. Frequent tube reaming is necessary if plugging cannot be avoided. Adoption of thick wall tubing like $5 / 16^{\prime \prime} \times .064 "\left(0.1845^{\prime \prime}\right.$ ID) and the arrangement as shown in Figure 48, may help ease the problem. Natural convection caused by a large temperature difference around the tube could aid the forced convection by this arrangement and oppose it by positioning the tube below the heater element. Although thicker tube walls provide higher conduction resistance, the controlling factor in heat transfer rates is determined by the fluid instead of the metal walls.

\section{Internal Electric Heating}

The problem of the uneven temperature distributions may be solved by the following arrangement. A large electrical current is applied directly across the part of the tube to be heated. The electric power is dissipated in the stainless steel tubing which acts as a resistance element. The longer the heating section, the lower the amperage and the higher the voltage. Both ends of the tubing are silver-soldered to copper blocks as shown in Rreith et al 25 The copper blocks on both ends are connected to the current source. The rest of the flow loop is protected and electrically insulated at the two connections to the heating section by special insulation washers. 
Drilling fluids are wore or less electrolytic liquids. Their electrical resistivities are probably of the same order as sea water, 1.e. 21 ohm-cm, compared to $74 \times 10^{-6}$ ohm-cm of stainless steel. The extreme difference indicates most of the current would be carried by the metal, not by the electrolyte. It is, therefore, feasible to adopt this setup, espectally for shorter heating lengths such as 10 feet for protection and control reasons.

Longer lengths are applicable only when the joints between tubes are well soldered, to permit good electrical conduction between them. This arrangement has not been adopted because of difficulties in applying it to longer heating length.

4. Eeat Transfer Liquids

The use of heat transfer liquid has the advantage of providing a uniform temperature distribution for the mud to be heated. Its disadvantage is the addittonal complications of the circuit for the heat transfer liquid.

A choice among heat transfer liquids such as liquid metals, organic and Inorganic salts was considered. An Inorganic salt mixture called Eitec (e.1. Du Pont De Nemours \& co. Inc.) was selected for this application because of the following merits:

a, Negligible vapor pressure at operating temperature, which permits its use in an atmospheric system or open bath.

b. Superior thermal properties for heat transfer. 
c. Stable and nontoxic at high temperatures.

d. Low corrosion to steel and many other popular metals.

A problem assoclated with Hitec is caused by its relatively high melting point $\left(288^{\circ} \mathrm{F}\right.$ in pure state). Fully covered insulation should be provided to prevent it from being solidized. Exposure to the ambient temperature and the presence of joints and fittings, which are the causes of local hot spots and potential leaking, can be reduced by adopting the least-exposed shell of a single large-diameter cylinder for salt accomodation.'A vertical arrangement of the cylinder is helpful in improving natural convection in the salt side of the heat exchanger and in simplifying fabrication. Natural convection in such a closed-end enclosure has been studied extensively in thermosyphon systems.1930 The natural convection which plays an important role in heat transfer due to excellent thermal properties, could be aided by the external forces provided by either of the following methods:

a. Circulation in the cylinder is imparted by a gear pump which is installed at the bottom. A 3 " tube is led from the discharge end of the pump to the salt bath, where a manfold is used to prevent channelizing of the flow. Circulation is alded by an axial-flow agitator attached to a motor shaft which drives the pump at the bottom. The internal circulation removes the heat from the electrical elements located around the inside 
circumference of the cylinder and transmits it to the tube bundle near the center. Some uncertainties as soclated with this arrangement are:

1. The forced convection may not aid but oppose the natural convection, because the latter is a complex phenomenom influenced by several parameters, as discussed in Chapter IX.

2. Channelizing of the circulation cannot be eliminated and it reduces efficiency of the downward flow in the central core, (Fig.50).

3. The pressure created by the agitator is usually Iimited to a few psi, which is not sufficient to overcome the high pressure drop across the tube bundle. The local velocity far away from the manifold would become too low. The turbulence produced by its motion may also destroy the delicate pattern of the natural convection.

4. The circulation is essentially parallel to the boundary layer near the tube walls. Lack of radial convection restricts the efficiency of heat transfer.

Some improvements can be made by the following arrangement:

b. A smaller cylinder such as a 2 " pipe with both ends sealed is placed in the vertical cylinder. A few tubes along with the same number of immersion heating elements are installed alternately around the inner 
cylinder in a circle with a small clearance from its surface. As shown in Figure 5l, a velocity fleld is generated by the rotation of the cylinder which is driven by a fractional horsepower motor at the top of the stationary sleeve. The cross flow to the mud tubes and heater elements greatly enhances heat transfer. This arrangement shows the advantages in simplicity and efficlency. Attachment of a few "low" fins on the cylinder surface further promotes the convection, with an increase in motor rating. Among the above arrangements, only the external electric heating and the rotating cylinder units are considered, along with the mud-to-mud heat exchanger. They will be discussed in more detail later and be compared in Table 22. 
CHAPTER VI

\section{EVAIUATION OF THERIMAL PARAMETERS OF DRIILING FLUIDS}

Heat capacity and thermal conductivity are two major thermal parameters in heat transfer. Their variations with temperature are relatively insignificant in the design calculations. Data on the thermal properties of drilling fluids is lacking in the literature. Some assumptions need to be ade for estimation of these parameters used in the heat transfer calculations. Some established correlations for suspensions of inactive particles are available:

1. Heat capacity of a suspension liquid is the weighted average of the values for the individual components. 2939 This statement fits better for the suspensions consisting of components with nearly equal heat capacities or negligible amounts of the otherwise dissimilar-valued components. For example, heat capacities of water, barite and bentonite are $1.004,0.113$, and $0.224 \mathrm{BTU} / 1 \mathrm{~b}^{\circ} \mathrm{F}$ respectively. The $12 \mathrm{ppg}$ base mud with $4 \%$ bentonite yields a value of $0.646 \mathrm{BTU} / \mathrm{Ib}^{\circ} \mathrm{F}$. Other examples for different und weights are compared in Table 21. Thermal parameters of the major constituents in drilling muds are shown in Table 20 . The contributions made by other additives in the results are small due to negligible amounts in the muds. 
TABLE 20

THERMAL PARAMETERS OF MAJOR CONSTITUENTS IN DRIILING FIUIDS

\begin{tabular}{|c|c|c|}
\hline Constituent & $\begin{array}{c}\text { Reat Capacity } \\
\text { BTU/1b } F\end{array}$ & $\begin{array}{c}\text { Thermal Conductivity } \\
\text { BIU/hft }{ }^{\circ} F\end{array}$ \\
\hline Barite & $0 . i 13 @ 208^{\circ} \mathrm{F}$ & $0.872 @ 212^{\circ} F(11)$ \\
\hline Bentonite & 0.224 & \\
\hline Water & $1.004 @ 200^{\circ} \mathrm{F}$ (24) & $0.393 @ 200^{\circ} \mathrm{F}(24)$ \\
\hline
\end{tabular}

TABLE 21

COMPARISON OF CAICULATED THERMAL PARAMETERS OF DRIIIING FLUIDS . BASED ON MUD WEIGHT (For $20 \mathrm{gph}$ Flow Rate)

\begin{tabular}{|c|c|c|c|}
\hline $\begin{array}{c}\text { Mud Weight } \\
\text { WPg }\end{array}$ & $\begin{array}{c}\text { Heat Capacity } \\
\text { Cp } \\
\text { BTU/1b }{ }^{\circ} F\end{array}$ & $\begin{array}{c}\text { W } \times \mathrm{Cp} \times 20 \\
\text { BTU/h }{ }^{\circ} \mathrm{F}\end{array}$ & $\begin{array}{c}\text { Thermal Conductivity } \\
\mathrm{K} \\
\text { BTU/hft }{ }^{\circ} \mathrm{F}\end{array}$ \\
\hline 9 & 0.914 & 164.5 & 0.401 \\
12 & 0.646 & 155.0 & 0.447 \\
15 & 0.485 & 145.5 & 0.489 \\
18 & 0.377 & 113.1 & 0.544 \\
\hline
\end{tabular}


2. Theral conductivity of a binary suspension of inactive particles is expressed below: ${ }^{29}$

$$
k_{5}=k_{1} \frac{2 k_{1}+k_{p}-2 x_{v}\left(k_{1}-k_{p}\right)}{2 k_{1}+k_{p}+x_{v}\left(k_{1}-k_{p}\right)}
$$

where $k_{s}, k_{l}, k_{p}$, are the thermal conductivities of the suspension, Iiquid phase and suspended particles respectively and $x_{v}$ is the volume fraction of solid in the suspension.

3. Thermal expansion plays a significant role in natural convection problems. The coefficients of thermal expansion of suspensions of inactive particles can be approximately. derfved from those of base liquids by assuming negligible changes in the volume of solid particles with temperature:

$$
B_{m}=B_{w}\left(1-x_{v}\right)
$$

where $B_{m}$ and $B_{w}$ are the coefficients of thermal expansion of the mid and water respectively, $x_{v}$ is the volume fraction of solid particles in the mud.

4. Capacity rate is the product of heat capacity and mass flow rate. As shown in Table 21 , capacity rates of weighted muds decrease with mud weight. Therefore, the design calculations will be based on lower mud welght of 9 ppg.

The above theoretical relations for fnert suspensions are not necessarily valid for drilling fluids of reactive particles. Experiments should be conducted if ectual thermal parameters need to be known. 
TABLE 22

COMPARISON OF HEAT IRANSFER UNITS EMPLOYED

\begin{tabular}{|c|c|c|c|c|}
\hline & $\begin{array}{l}\text { Mud-to-Mud } \\
\text { Exchanger }\end{array}$ & $\begin{array}{c}\text { Electrical } \\
\text { Heater }\end{array}$ & $\begin{array}{l}\text { Molten Salt } \\
\text { Exchanger }\end{array}$ & $\begin{array}{l}\text { Water } \\
\text { Cooler }\end{array}$ \\
\hline $\begin{array}{l}\text { Unit } \\
\text { Arrangement }\end{array}$ & $\begin{array}{l}2 \text { parallel } \\
\text { tubes silver } \\
\text { soldered side } \\
\text { by slde }\end{array}$ & $\begin{array}{l}\text { Tubes silver } \\
\text { soldered with } \\
\text { electrical } \\
\text { tubular } \\
\text { elements }\end{array}$ & $\begin{array}{l}\text { Rotating } \\
\text { cylinder with } \\
\text { molten salt } \\
\text { in the } \\
\text { annulus }\end{array}$ & $\begin{array}{l}\text { Annular } \\
\text { water } \\
\text { jacket }\end{array}$ \\
\hline $\begin{array}{l}\text { Terminal Termp } \\
\text { of the fluid }\left({ }^{\circ}\right)\end{array}$ & $115-300$ & $300-550$ & $375-550$ & $365-115$ \\
\hline $\begin{array}{l}\text { Calculated } \\
\text { Heat Requirement } \\
\text { (BTU/h) }\end{array}$ & 30,433 & 41,250 & 28,875 & 41,125 \\
\hline $\begin{array}{l}\text { Heat Loss From } \\
\text { the unit (BTU/h) }\end{array}$ & $\begin{array}{c}3800 \\
\text { (uninsulated) }\end{array}$ & $\begin{array}{c}800 \\
\text { (insulated) }\end{array}$ & 1500 & -- \\
\hline $\begin{array}{l}\text { Designed } \\
\text { Heat Requirement } \\
(B T U / h)\end{array}$ & 34,233 & 42,050 & 30,375 & 41,125 \\
\hline $\begin{array}{c}\text { Medium } \\
\text { Heat Transfered } \\
\text { With }\end{array}$ & $\begin{array}{l}\text { Drilling } \\
\text { Mud }\end{array}$ & $\begin{array}{l}\text { Electrical } \\
\text { tubular } \\
\text { elements }\end{array}$ & Hitec & Water \\
\hline $\begin{array}{l}\text { Terminal Temp } \\
\left.\text { of the medium }{ }^{\circ} F\right)\end{array}$ & $550-365$ & About 650 & About 750 & $78 \div 92$ \\
\hline $\begin{array}{c}\text { Convective } \\
\text { Film Coefficlent } \\
\left(B T U / h f t^{2}\right.\end{array}$ & 112 & 101 & 160 & 112 \\
\hline $\begin{array}{l}\text { Overall Film } \\
\text { Coefficient } \\
\text { (BTU/hft }\end{array}$ & $59^{\star \star}$ & --- & $133 * *$ & $77 * *$ \\
\hline $\begin{array}{l}\text { Required } \\
\text { Length }(f t)\end{array}$ & 49 & $64 *$ & 30 & $95^{\star \star}$ \\
\hline
\end{tabular}

Based on $75 \%$ of the maximum wattage

** Assuming fouling factor of $0.001 \mathrm{hft} t^{\circ} \mathrm{F} / \mathrm{BTU}$

*** Based on a flow rate of $6 \mathrm{gpm}$ 
CHAPTER VII

SIZING OF TEE MUD-TO-MDD BEAT EXCAANGER

The system requires heating and cooling of the test fluid repetitively. The optimum design is to take advantage of the heated returning mud to heat the cool advancing mud, in the view point of power consumption. Both streams have nearly equal capacity rates. It is generally a good practice to have both capacity rates equal if they are not too small to retard the heat transfer. In the NTU (Number of Transfer Units) method of heat exchanger design, effectiveness, which is the ratio of actual heat transfer rate to the thermodynamically maximu possible heat transfer rate, shows 50\% for unity NTU. 21 To determine the outlet temperature of the mudto-mud exchanger, it is reasonable to assume $50 \%$ effectiveness, beyond which the effectiveness curve starts to approach an asymtotical limit. That means an additional length of transfer units has less efficiency and increasing pumping pressure. To shorten the startup time, it is also desirable to limit the length of this section, from the heat balance:

$$
W c_{p}\left(t_{2}-t_{1}\right)=W^{\prime} c_{p}^{\prime}\left(t_{1}^{\prime}-t_{2}^{\prime}\right)
$$

The inlet temperatures $t_{1}, t_{1}^{\prime}$, of the two streams are known to be $110^{\circ} \mathrm{F}$ and $550^{\circ} \mathrm{F}$ respectively. The effectiveness $50 \%$ represents outlet temperatures of $330^{\circ} \mathrm{F}$ in both streams.

Next, to find convective filn coefficients of drilling fluids, 
Oliver and Jenson's empirical correlation for power law fluids in horizontal tubes is used $:^{36}$

$$
\frac{\bar{h} d}{k}=1.75\left[\frac{W C_{p}}{k L}+.0083\left(\frac{C_{p} \mu_{a}}{k} \frac{B g \rho^{2} d^{3} \Delta t}{\mu_{a}^{2}}\right) 0.7\right) 1 / 3\left(\frac{\gamma_{b}}{\gamma_{w}}\right)^{0.14}
$$

where $\bar{h} d / k$ is the mean Nusselt number, WC $/ \mathrm{kL}$ the Graetz number, $C_{p} \mu_{a} / k$ the Prandt 1 number, $B g \rho^{2} d^{3} \Delta t / \mu_{a}{ }^{2}$ the Grashof number and the fluid consistency $\gamma=K\left(\frac{3 n+1}{4 n}\right) \cdot 8^{n-1} \cdot 15$ The first term on the right-hand side represents forced convection, while the second term natural convection. The ratio $\gamma_{b} / \gamma_{w}$ allows correction for the variation of viscosity with temperature. Natural convection usually plays a significant role in flow at relatively low velocities. Large temperature differences and large unit dimensions are indicated by the Grashof number. It is necessary to evaluate the relative gnitudes of the two convections for different flow rates, viscosities and mud weights. From the dimensional analysis of the NavierStokes equation, the ratio of $\mathrm{Gr} / \mathrm{Re}^{2}$ ( $\mathrm{Gr}$ is the Grashof number, $\operatorname{Re}$ the Reynolds number) is found to be a qualitative indicator of the significance of the buoyancy force in heat transfer ${ }^{13}$. The ratio $\mathrm{Gr} / \mathrm{Re}^{2}$ is equal to $\left[\left(\pi^{2} / 16 g_{C}\right) \cdot\left(B g d{ }^{5} \Delta / Q^{2}\right)\right]$ from which it shows that viscosity and mud weight play no roles in determining the relative significance of the two convections. The diameter and flow rates have profound influence on the contribution of natural convection. Table 23 , Indicates negligible natural convection in the presence of forced convection except for the cases of low flow rates and large temperature differences between the fluld and the wall according to whether $G r / \operatorname{Re}^{2}$ is of the order of one. Precaution should be taken for the probable high local temperature on the tube wall close to the connected electrical heater element. Onission of buoyancy forces will lead to 
overestimation and yield more conservative results. Ignoring the natural convection, the equation becomes:

$$
\frac{\overline{\mathrm{h}} \mathrm{d}}{\mathrm{k}}=1.75 \Delta^{1 / 3}\left(\frac{\mathrm{WC}}{\mathrm{k} \mathrm{L}}\right)^{1 / 3}\left(\frac{\gamma_{\mathrm{b}}}{\gamma_{\mathrm{W}}}\right)^{0.14}
$$

where $\Delta^{1 / 3}$ is a constant depending on flow behavior index $n$, as given in Skelland. 36 There are three unknowns in this equation, the average film coefficlent $\bar{h}$, total heated length $L$, and wall temperature $t_{w}$ at which $\gamma_{w}$ is given. Solution of these unknowns calls for trial and error. For the mud-to-mud exchanger, $t_{w}$ is approximated by the average of the temperatures of both streams because of the symetilc geometry and equal capacity rates. One restriction of using Equation 3 is the condition of a uniform temperature along the tube wall. The varying wall temperature in the mud-tomud exchanger requires an iteration scheme accomplished by using digital computer:

(1) Divide the required heat flux $q$ and the range between the outlet and inlet temperatures $t_{2}-t_{1}$ into $n$ segments in each of which temperature is considered uniform, and Eq. (3) becomes applicable.

(2) Assume a total length $L$ of the heat exchanger.

(3) Let $I^{\prime \prime}=0$

(4) Evaluate the average temperatures of both streams and at the tube wall in each temperature segment by starting from the the inlet temperature of the cold stream and the outlet temperature of the hot stream.

(5) Calculate $\gamma_{b}^{\prime} / \gamma_{W}$ from the power law parameters $K$ and $n$ which are interpolated by cubic spline.

(6) Calculate the Graetz number $W C_{p} / k I$. 
(7) Calculate the film coefficients $h$ and $h$ ' by Eq. (3).

(8) Calculate the overall film coefficient $U$ by:

$$
1 / 0=1 / h+A / A^{\prime} h^{\prime}+x_{w} A / k_{w} A_{w}+R_{E}
$$

where $R_{f}$ is the thernal resistance produced by fouling (Chapter VIII).

(9) Determine the logarithmic mean temperature difference (IMID) by :

$$
\operatorname{IMTD}=\frac{\left(E_{1}^{\prime}-t_{2}\right)-\left(t_{2}^{\prime}-t_{1}\right)}{\ln \frac{\left(t_{1}^{\prime}-t_{2}\right)}{t_{2}^{\prime}-t_{1}}}
$$

with $t_{1}, t_{2}, t_{1}^{\prime}, t_{2}^{\prime}$, replaced by the terminal temperatures of the temperature segment. For the mud-to-mud exchanger, LMTD is just $t_{2}^{\prime}-t_{1}$.

(10) Calculate the required length L for the temperature segment by :

$$
L_{I}=\frac{g / n}{\pi d U \cdot \operatorname{LMTD}}
$$

(11) Let:

$$
\begin{aligned}
& I^{\prime}=L^{\prime}+L \\
& L_{t}=L^{\prime} n / i
\end{aligned}
$$

where $i$ is iteration number of the 1th temperature segment.

(12) Replace the $L$ in Step (2) by $\left(L+L_{t}\right) / 2$ and repeat the calculation loop starting from step (6) until the difference $L-L_{t}$ is sufficiently small.

(13) Let $L^{\prime \prime}=I^{\prime}$. 
(14) Proceed to the next temperature segment and repeat the calculation from Step (2) with L obtained from Step (12) in the last iteration.

(15) Print $L^{\prime \prime}$ which is the final result after all temperature segments are calculated.

The flow chart of the above scheme is shown in Appendix A.

\section{TABLE 23}

SIGNIFICANCE OF NATURAI CONVECTION BASED UPON THE GR/RE ${ }^{2}$ RATIO

\begin{tabular}{|c|c|c|}
\hline Flow Rate & $\begin{array}{c}50^{\circ} \mathrm{F} \\
(8 \mathrm{cp})\end{array}$ & $\begin{array}{c}200^{\circ} \mathrm{F} \\
(8 \mathrm{cp})\end{array}$ \\
\hline $20 \mathrm{gph}$ & $6 \times 10^{-3}$ & $3 \times 10^{-2}$ \\
\hline $8 \mathrm{gph}$ & $4 \times 10^{-2}$ & $1.6 \times 10^{-1}$ \\
\hline
\end{tabular}

Table 5 shows the results for a particular mud data. The effects of mud parameters and cirçulation rates on the heat transfer rate and the required heating length were investigated. The calculated film coefficient $h$ ranges from 53 to $250 \mathrm{BTU} / \mathrm{h} \mathrm{ft}^{2} \mathrm{~F}$ and the theoretical length requirements scatter from 58 ft to 43 ft. Because the standard deviation of Eq. (3) was claimed to be about $15 \%$, safety factor $15 \%$ may be added to yield 56 ft.

The ratio of wall thickness to the mean wall area $x_{w} / A_{w}$ in Eq. (4) needs to be estimated for the geometry of two brazed tubes in the mud-tomud exchanger. Since the thermal conductivity of silver solder (2IO BTU/h $\left.\mathrm{ft}^{\circ} \mathrm{F}\right)$ is considerably higher than that of stainless steel $\left(10 \mathrm{BTU} / \mathrm{h} \mathrm{ft}{ }^{\circ} \mathrm{F}\right)$, the actual geometry can be approximated by a simple model of flat surface 
TABLE 24

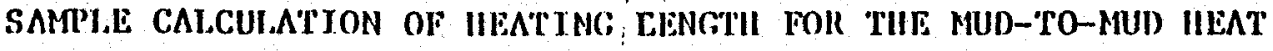
EXCIIANGFIR

\begin{tabular}{|c|c|c|c|c|c|c|c|c|c|c|c|c|}
\hline Temr & N & 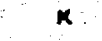 & APP.VIS & COAA. & COnA" & 11 & Non & $\mathbf{u}$ & LENGTH & WALL TEMP & AEN & PAL \\
\hline 186.333 & 0.040 & 0.091 & 12.644 & 1.031 & 1.049 & $107 \cdot 033$ & 117.014 & 21.312 & 3.412 & 241.333 & 800.228 & 0.360 \\
\hline 129.000 & 0.037 & 0.067 & 11.003 & 0.906 & 1.013 & 102.576 & 113.182 & 99.211 & 3.803 & 254.000 & 533.040 & 0.349 \\
\hline 141.667 & 0.033 & 0.004 & 10.962 & 0.5 .73 & 1.000 & 101.081 & 182.470 & 49.730 & 3.630 & 268.687 & 976.969 & 0.321 \\
\hline 159.933 & 0.016 & 0.008 & 0.748 & 0.964 & 1.006 & 100.620 & 111.040 & 40.533 & 3.696 & 279.333 & 405.207 & $0.20 \mathrm{r}$ \\
\hline 167.000 & 0.009 & 0.005 & 0.104 & 0.035 & 0.900 & 09.ess & 108.707 & 47.160 & 3.751 & 292.000 & 600.684 & 0.271 \\
\hline 170.047 & 0.000 & 0.005 & 0.020 & 0.983 & 0.975 & 90.626 & 106.063 & 47.320 & 3.730 & 304,666 & 733.081 & 0.253 \\
\hline 192.333 & 0.790 & 0.006 & 0.072 & 0.056 & 0.000 & $100 \cdot 24 !$ & 100.730 & 47.030 & 3.699 & 317.333 & 763.521 & 0.240 \\
\hline 208.000 & 0.006 & 0.000 & 7.254 & 0.033 & 1.003 & $97 \cdot 130$ & 110.353 & $4 T \cdot 440$ & 3.729 & 329.909 & $\operatorname{ar1} 1.092$ & 0.214 \\
\hline 217.067 & 0.790 & 0.073 & 0.861 & 0.943 & 1.018 & 00.747 & 112.435 & 90.102 & 3.672 & 342.066 & 921.900 & 0.204 \\
\hline 230.333 & 0.772 & 0.079 & 6.460 & 0.950 & 1.027 & 100.243 & 113.908 & 40.023 & 3.624 & 355.332 & 077.629 & 0.103 \\
\hline $243,0<0$ & 0.794 & 0.087 & 6.077 & 0.954 & 1.033 & 101.300 & 1180001 & 49.323 & 3.507 & 367.998 & 1000.764 & 0.103 \\
\hline 285.667 & 0.684 & 0.129 & 0.217 & 0.808 & 1.038 & 100.291 & 116.258 & 81.009 & 3.463 & 300.663 & 1212.302 & 0.162 \\
\hline 260.332 & 0.073 & 0.837 & 0.100 & 0.091 & 1.044 & 109.240 & 118.308 & 81.627 & 3.434 & $393 \cdot 332$ & $120 c .071$ & 0.159 \\
\hline 201.004 & 0.682 & 0.147 & 4.908 & 0.095 & 1.040 & 110.234 & 128.400 & 81.065 & 3.408 & 409.898 & 1280.286 & 0.187 \\
\hline 293.606 & 0.081 & 0.127 & 9.064 & 1.033 & 1.000 & 115.177 & 123.731 & 64.080 & 3.273 & 410.663 & 1200.207 & 0.184 \\
\hline
\end{tabular}

TOTAL LENGIHE aJ.T REET

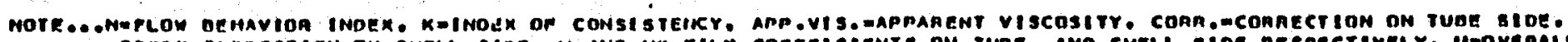

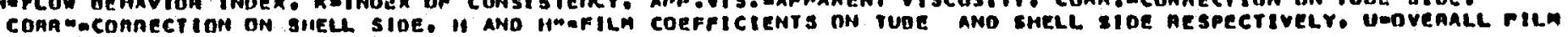

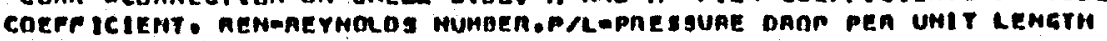


with thickness equal to twlce the tube wall thickness and width equal to the width of silver solder between the contacting tubes, assuming infinite thermal conductivity of silver solder (Figure 49). More exact solution for the conduction resistance of the actual geometry can be obtained by the finite difference method. 1 The probable error caused by adopting the simplified model is not serious because the overall heat transfer rate is controlled by the convective film instead of the conduction resistance. The model indicates that increasing soldering thickness will help conduction somewhat. Wrapping the two brazed tubes by copper foils may also enhance conduction and reduce excessive local heat flux density to some extent. However, considerable improvements may not be expected by such changes.

As shown in Figure 49 with an inclined arrangement as in Figure $48(\mathrm{c})$ each stream is heated or cooled from one side of the tube. The hotter layers near the wall become less dense than the bulk fluld and tend to rise, thereby, distorting the flow pattern and affecting the heat transfer. The relatively cooler layers in another tube show identical but opposite flow pattern. This configuration takes advantage of the buoyant forces.

The fouling factor $R_{f}$ in Eq. (4), is an unknown for drilling fluids because it depends on flow velocity, wall temperature and fluid composition etc. An empirical value of $0.003 \mathrm{~h} \mathrm{ft} \mathrm{C}^{\circ} \mathrm{F} / \mathrm{BTU}$ for muddy water was considered the calculations. 12 If the tubes are reamed often during operations, a value of $0.001 \mathrm{~b} \in \mathrm{t}^{2} \mathrm{O}_{\mathrm{F} / \mathrm{BTU}}$ may be used instead. 
FIGURE 40

SIMPLIFIED MODEL OF THE BRAZED PARALIEI-TUBES
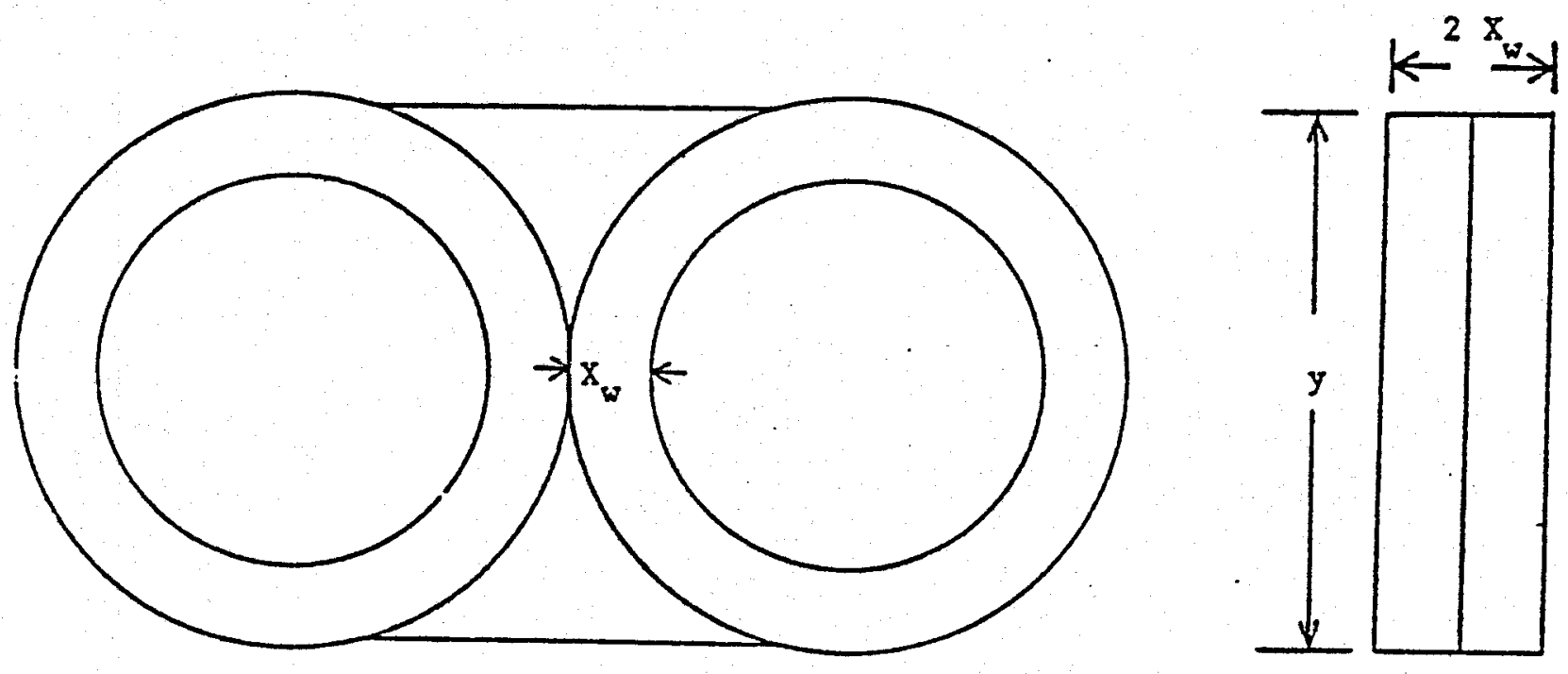
CHAPTER VIII

\section{SIZING OF THE ELECTRICAL HEATING SECTION}

From the energy balance, the heat generated by the electric current through the resistance wire should transmit to the mud in the parallel-brazed tube, assuming negligible heat loss through insulation and viscous dissipation energy of the mud. We have:

$$
q_{e}=W C_{p}\left(t_{2}-\zeta_{1}\right)
$$

The heat flux required to heat the mud from $t_{1}=330^{\circ} \mathrm{F}$ to $t_{2}=550^{\circ} \mathrm{F}$ is equivalent to $10 \mathrm{kw}$ and $8 \mathrm{kw}$ for flow rates $20 \mathrm{gph}$ and $15 \mathrm{gph}$ respectively. The surface temperature of the electrical heater should be known for estimating heat losses from the insulated tubes. From the following equation:

$$
q_{e}=q_{e}=\overline{h A}\left(t_{w}-t_{b}\right)
$$

the temperature difference $t_{w}-t_{b}$, is estimated to be about $120^{\circ} \mathrm{F}$ for film coefficient $h$ of $104 \mathrm{BTU} / \mathrm{h}$ ft ${ }^{\circ} \mathrm{F}$. The average temperature of the mud tube is $(330+550) / 2$ or $440^{\circ} \mathrm{F}$. The overall mean temperature of the soldered pair is therefore, about $500^{\circ} \mathrm{F} .{ }^{4}$ The heat loss per unit area is found from the heat transfer equation for cylindrical surfaces:

$$
q_{1}=\frac{t_{1}-t_{0}}{\frac{r_{0}}{k_{1}} \ln \frac{r_{0}}{r_{1}}+R_{2}}
$$

where $t_{i}$, $t_{0}, I_{i}$, $I_{0}$, are the temperatures and radii of the inside and 
outside surface of the insulation respectively, $k_{i}$ thermal conductivity of the insulation and $R_{a}$ ' convective resistance of the air. Using the following data:

$$
\begin{aligned}
& r_{i}=.42^{\prime \prime}, r_{0}=1.98^{\prime \prime} \text { (for } 1 \frac{1}{\prime \prime} \text { nominal thickness of insulation) } \\
& k_{i}=.52 \mathrm{BTU} / \mathrm{h} \mathrm{ft}{ }^{2} \mathrm{~F} / \text { inch (for calcium silicate at } 500^{\circ} \mathrm{F} \text { ) } \\
& R_{a}=.55 \mathrm{~h} \mathrm{ft}{ }^{2}{ }^{\circ} \mathrm{F} / \mathrm{BTU} \text { (McAdams, } 27 \mathrm{p} .179 \text { ) }
\end{aligned}
$$

the heat loss per unit area is found to be $15.1 \mathrm{BTU} / \mathrm{h} \mathrm{ft}^{2}$. Total heat Ioss for the 50-foot length of heating section is estimated to be 3375 BTU/h or $1.0 \mathrm{kw}$. The overall wattage requirements are 8.5 and $6.7 \mathrm{kw}$ for flow rates $20 \mathrm{gph}$ and $15 \mathrm{gph}$ respectively. The former requires eight heater elements, the latter just six.

The average heat flux density created by the heater element on the soldered side of the tube periphery is estimated approximately according to Figure 49 . The heater elements have the following specifications:

$$
\begin{aligned}
& \text { Wattage }=1965 \text { watts } \\
& \text { Heated length } L=89.5^{\prime \prime} \\
& \text { Diameter }=0.246^{\prime \prime}
\end{aligned}
$$

If the soldered width $y$ is $95 \%$ of the element diameter, $1 . e .0 .234^{\prime \prime}$, the heated area on the outside boundary of the tubing is $\mathrm{Ld} \sin ^{-1}(\mathrm{y} / \mathrm{d})=27.1$ sq. In. Heat flux density is therefore, $1965 / 27.1=72.5$ watts/sq. in. Comparing with 55.9 watts/ sq. In. for $\mathrm{y}=0.25^{\prime \prime}$, there is an improvement of about $23 \%$ in reducing heat flux density.

\section{ASSOCIATED FOULING PROBLEMS}

Fouling refers to the undesirable deposit on heat exchanger 
surfaces which increases resistance to heat transmission. There are a few fouling mechanisms most frequently occured - crystallization, sedimentation, coking and others. ${ }^{37}$ Drflling fluids are usually suspensions contatiing a rather high concentration of a large variety of salts. Salts of inverse solubility character, exhibiting decreasing saturation concentration with temperature beyond certain temperature, are often blamed for fouling due to their crystallization (such as $\mathrm{CaSO}_{4}, \mathrm{CaCO}_{3}$ ). In suspensions, sedimentation of suspended particles accounts for many fouling problems, especially in the cases of low flow velocities. Therefore, drilling fluids are very susceptible to fouling particularily in the case of high heat flux density, under which crystallization of salts may become excessive. As shown in Chapter XII, the mud pressure in the electrical heating section, due to large pressure drop in the tube, may become insufficient to prevent boiling at least in the localized area near the high-flux boundary. Local boiling, superimposed by the above mentioned crystallization and sedimentation, can make fouling or plugging a sertous problem.

There are some precautions which may help lessen the plugging problem somewhat, in addition to the arrangements, mentioned in Chapter V:

(1) Cover the largest possible area between the tubing and heater element with silver while soldering.

(2) Install twisted tapes in the tube bore to produce swirling flow in the tube ${ }^{12}$ and to help remove heat from the hot spots.

(3) Overdesign the required length and, during operations, turn down the heater wattage.

(4) Adopt triangular heater elements instead of tubular elements, because more surface area per unit volume of the refactory 
around the resistance wire is avallable and can provide more welding surfaces.

(5) Mintain high flow velocity and high pump pressure.

(6) Install such turbulence promotors in the tube bore as spiral wires, twisted strips or wavy rods ${ }^{34}$ to reduce the thermal boundary layer at the tube wall which is the major resistance to heat transfer and, in turn, the probable cause of plugging problems. 
CHAPTER IX

\section{SIZING OF THE MOLTEN SALT HEAT EXCHANGER}

The heat transfer in a vertical salt bath without external mechanical forces is an "internal flow" problem of natural convection. The type of flow in this case depends on the height-radius ratio $1 / 5$ for fixed Prandtl number $P r$ and Grashof number. The Rayleigh number is $R_{a}+G_{T} \times P_{T}$. For relatively large $I / r$, the upward boundry layer flow around the inside wall of the cylinder is coupled by the downward flow of the central core (Figure 23 ). Large value of ( $R a$ ) (r/1) generally indicates large heat transfer between the cylinder wall and the cold tube surfaces, by means of the boundary layer flow. The thickness of the relatively thin boundary layer tends to keep constant along the axial direction except near both ends. To evaluate the significance of natural convection, the Grashof number and the Rayleigh number Ra should be known first. The term $\Delta t^{\prime}$ in the Grashof number is unknown and must be estimated by trial and error. From Table 5, which sumarizes the parameters of the molten'salt, Gr/ $\mathrm{t}^{\prime}$ is found to be $4.85 \times 10^{5}{ }^{\circ} F^{-1}$ for a $6^{\prime \prime}$ pipe. Martin ${ }^{26}$ and Japiksa correlated experimental data for open thermosyphons. Thelr conclusions indicate the Nusselt number Nu applied to our case will be close to 46 . It corresponds to a film coefficient $\mathrm{h}$ of around $40 \mathrm{BTU} / \mathrm{h} \mathrm{ft} \mathrm{f}^{\circ} \mathrm{F}$. This somewhat low value suggests the need of mechanical aid to enhance heat transfer. Sizing of the rotational arrangement mentioned in Chapter IV is attempted as follows. 
FIGURE 41

FLOW PATTERN OF NATURAL CONVEGTION

IN AN ENCLOSURE

HEATED FROM THE OUTSIDE

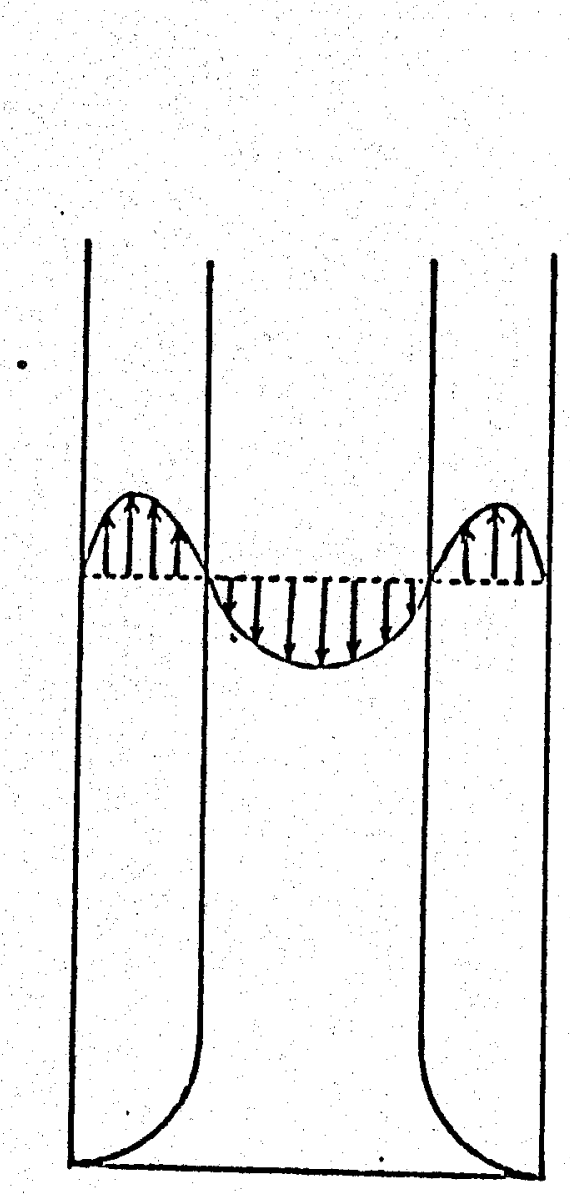

FIGURE 42

SCHEMATIC DIAGRAM OF THE ROTATING

CYLINDER HEAT EXCHANGER

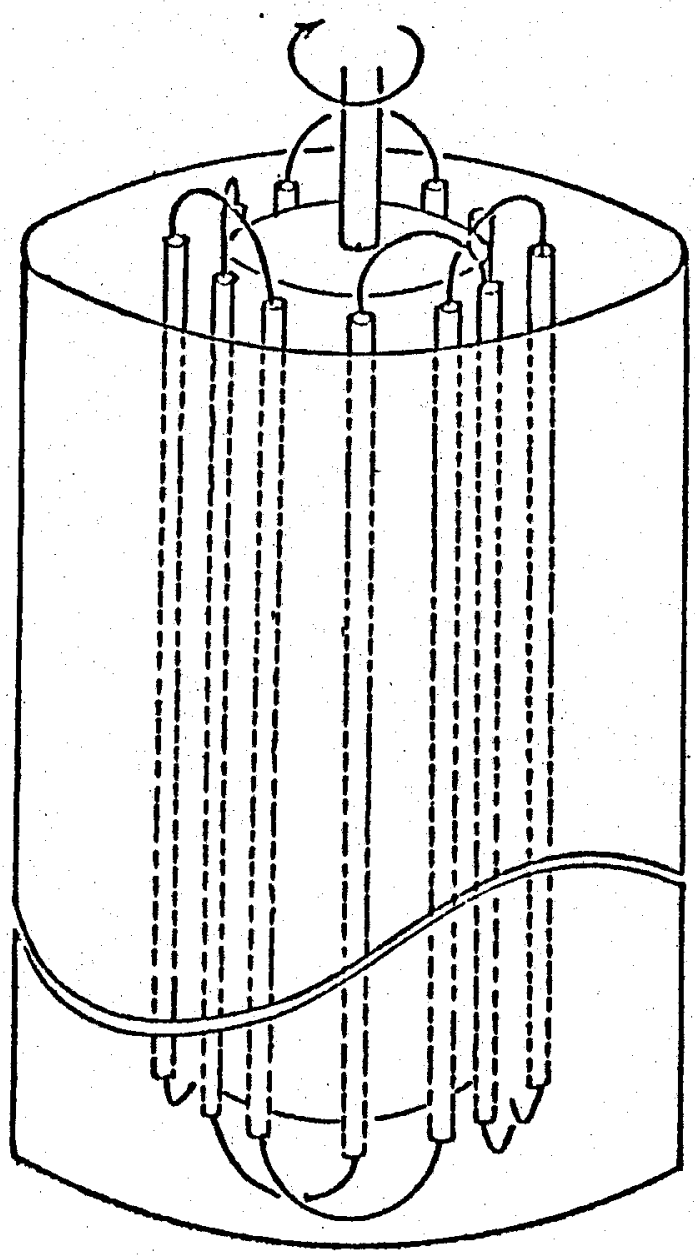


The velocity field produced by the rotating cylinder can be obtained by solving the Navier-Stokes equation for the laminar flow. ${ }^{42}$ The result is expressed as:

$$
v=\left(\frac{\omega R_{1}}{12}\right)\left(\frac{R_{1}\left(R_{2}^{2}-r^{2}\right)}{r\left(R_{2}^{2}-R_{1}^{2}\right)}\right)
$$

where $v$ is the tangential velocity at radius 5 . The raquired torque of the rotation is given by: 35

$$
\tau=1.823 \times 10^{-6} L_{\mu \omega} \frac{R_{1}{ }^{2} R_{2}{ }^{2}}{R_{2}{ }^{2}-R_{1}{ }^{2}}
$$

The theoretical horsepower requirement is:

$$
H P=\tau \omega / 550
$$

Combinining Equations (10), (11), and (12) yields:

$$
v=1447.58\left(\frac{R_{1}}{R_{2}}=\right) \frac{R_{2}^{2}-I^{2}}{\sqrt{R_{2}{ }^{2}-R_{1}{ }^{2}}} \cdot \sqrt{\frac{\mathrm{EP}}{L_{1}}}
$$

Cross flow velocity and convection can be increased by adopting a larger rotating cylinder and by allowing smaller clearance $R_{2}-R_{1}$ as indicated in Equation (13). Table 26 compares the results for different combinations of geometry and horsepower requirement. The distance $x$ between the consecutive tube and heater elements determines whether the downstream tube is affected by and in the wake of the upstream one. The experience from comercial cross flow shell-and-tube heat exchangers indicates that the closely spaced tube bundle promotes, turbulence and heat transfer considerably. The relatively small values of $x / d$ shown in Table 25 , indicate. probable influences of turbulent wakes, 10 although the even smaller ratio for comercial heat exchangers are usually not larger than 3.0 . Conservative 
estimation of the film coefficient can be made by using Zukauskar's correlation of the cross flow to a single tube: 43

$$
\frac{h d_{0}}{k}=0.26\left(\frac{d_{0} p v}{\mu}\right) 0.6\left(\frac{c_{p} \mu}{k}\right) 0.37
$$

for $1000<\operatorname{Re}<100,000$, where $v$ is the main stream velocity far away from the tubes and represents the velocity produced by the rotating cylinder. The fill coefficients are calculated and shown in Table 26. Following the same calculation procedure as for the mud-to-mud heat exchanger, the overall film coefficient $U$ is found to be $73 \mathrm{BTU} / \mathrm{h} \mathrm{ft}{ }^{2}{ }_{\mathrm{F}}$ and the required length of tube is $39 \mathrm{ft}$. for motor horse power of $1 / 50 \mathrm{HP}$. The considerably high values of velocities in Table 26 result from the low viscosity of Hitec and the smooth surfaces of cylinders. A few blades of fins can be attached on the cylinder surface to promote turbulence and reduce the required rotating speed.

TABLE 25

SOME PARAMETERS OF MOLTEN SALT AT $600^{\circ} \mathrm{F}$

\begin{tabular}{|c|c|c|c|}
\hline Density $\left(L B / F T^{3}\right)$ & 115.6 & Prandtl Number Pr & 12.2 \\
\hline $\begin{array}{l}\text { Heat Capacity } \\
(B T U / L B \\
F)\end{array}$ & 0.373 & Grashof Number Gr & $3 \times 10^{7}$ \\
\hline $\begin{array}{l}\text { Thermal Conductivity } \\
\left(B t u / h \in t^{\circ} F\right)\end{array}$ & 0.221 & Rayle1gh Number $R z$ & $3.7 \times 10^{8}$ \\
\hline Viscosity (cp) & 3.0 & Latent Heat (BTU/LB) & 35 \\
\hline $\begin{array}{l}\text { Thermal Expansion } \\
\left(\mathrm{E}^{-1}\right)\end{array}$ & $\begin{array}{l}2.31 x \\
10^{-4}\end{array}$ & Melting Point $\left({ }^{O} F\right)$ & 288 \\
\hline
\end{tabular}


As for the mechanical setup, it is simple in fabrication and easy for maintenance. The cylinder is driven by a motor with a conveyor belt. A ball bearing at the bottom of the stationary sleeve serves as support to the rotating axis. The weight of the sealed empty, inner cylinder is almost balanced by the buoyancy force of the liquid salt, which reduces the bottom support load.

TABLE 26

CALCULATED FIIM COEFFICIENT ON THE SHELL SIDE OF THE MOLTEN SALT BEAT EXCHANGER FOR DIFFERENT DIMENSIONS AND HORSEPOWER RATINGS

(BASED ON $L=5.5 \mathrm{FT}, R_{2}=3.03$ INCHES)

\begin{tabular}{|r|r|r|r|r|r|r|r|r|}
\hline$R_{1}$ & $\mathrm{HP}$ & $\begin{array}{c}\omega \\
(\mathrm{rpm})\end{array}$ & $\mathrm{I}$ & $\mathrm{x}=2 \pi \frac{\mathrm{I}}{12}$ & $\begin{array}{c}V \\
(\mathrm{ft} / \mathrm{sec})\end{array}$ & $\mathrm{x}+\mathrm{d}$ & $\mathrm{Re}$ & $\mathrm{h}$ \\
\hline 1.1875 & $1 / 60$ & 4084 & 1.5625 & 0.818 & 27.9 & 3.3 & $3.2 \times 10^{4}$ & 3512 \\
1.1875 & $1 / 50$ & 4475 & 1.5625 & 0.818 & 30.6 & 3.3 & $3.6 \times 10^{4}$ & 3770 \\
1.1875 & $1 / 40$ & 5003 & 1.5625 & 0.818 & 34.2 & 3.3 & $4.0 \times 10^{4}$ & 4016 \\
\hline 1.4375 & $1 / 60$ & 3229 & 1.8125 & 0.949 & 22.0 & 3.8 & $2.57 \times 10^{4}$ & 3080 \\
1.4375 & $1 / 50$ & 3537 & 1.8125 & 0.949 & 24.2 & 3.8 & $2.82 \times 10^{4}$ & 3256 \\
1.4375 & $1 / 40$ & 3955 & 1.8125 & 0.949 & 27.0 & 3.8 & $3.15 \times 10^{4}$ & 3480 \\
\hline
\end{tabular}

\section{SAFETY MEASURES}

Although no unusual safety hazards have been encountered by the industry using the salt, a few safety considerations should be made: 
1. A minimum number of fittings and valves are to be used.

2. Temperature measurement should be taken by means of remote indicating instruments.

3. The unit is best positioned at a location isolated from the operating floor area.

4. Foreign materials which may contaminate or corrode at high temperatures are to be removed before startup. Since Hitec is a strong oxidizing agent, all wood, paper or organic materials must be kept away from the salt. Only inorganic insulation is suitable.

5. Heating of the salt must be very gradual as it is likely to foam at $450^{\circ}$ to $500^{\circ} \mathrm{F}$. Totally it takes $10.7 \mathrm{kw}$. hr. to raise the temperature of the entire unit from room temperature to $750^{\circ} \mathrm{F}$. The required startup time would be $10.7 \mathrm{kw}$. hr. $/(6) \times 1.965 \mathrm{kw.})=: 55$ minutès. As long as two hours or more is needed to start up, if heating rate is reduced.

6. A small amount of water $(0.36 \mathrm{gal})$ is added to lower the melting point $\left(240^{\circ} \mathrm{F}\right)$ and to improve contact between the salt and tubular heaters. A relief valve at the top of the jacket provides outlet for the steam envolved as the salt is melted and heated.

7. Whenever possible, welded tube bends and connections should be used. Screwed fittings of the mud tubes may be found unsatisfactory, due to the extremely large temperature change during startup.

8. Every inch of the surface exposed to the ambient temperature 
is to be adequately Insulated; otherwise, the salt will freeze in thin films on the surface.

9. An over-temperature cutoff is installed near the top and close to the immersion heaters to shut off the unit whenever the temperature exceeds an arbitarily set maximum operating temperature.

10. An insulation joint between the rotating cylinder and the motor is used to keep the latter from being overheated.

11. The sleeve of schedule 40 carbon steel pipe must be carefully checked to see if it has sufficient strength to withstand pressure surges of 1000 psi caused by any accidental tube leaks. 
CHAPTER $\mathrm{X}$

\section{SIZING OF THE WATER COOLER}

Eeat transfer from the water flowing in the annular space between the 2 " pipe and $\frac{1}{2}$ " tube in laminar flow is estimated roughly by:

$$
\frac{\mathrm{h}^{\prime} \mathrm{d}_{\mathrm{e}}}{\mathrm{k}}=\left(\frac{W \mathrm{c}_{\mathrm{p}}}{\mathrm{kL}}\right)^{0.45}\left(\frac{\mathrm{d}^{\prime}}{\mathrm{d}_{0}}\right)^{0.8}(\mathrm{GI})^{0.05}
$$

where $d_{e}$ is equivalent diameter of the annulus equal to $d{ }^{\prime}-d_{o}$, Gr is the Grashof number allowing correction for the natural convection in low velocity flows. 27 The above equation fits better for small d'/do. In our case, $\mathrm{d}^{\prime} / \mathrm{d}_{0}$ is as large as 8.3. There are errors introduced in estimating the equivalent diameter $d_{e}$ and applying the above equation. The above equation is used, however, in the absence of empirical correlations for large annuli The film coefficient $h^{\prime}$ is $367 \mathrm{BTU} / \mathrm{h} \mathrm{ft}{ }^{2}{ }^{\circ} \mathrm{F}$, based on the flow rate of 6.0 gpm and the Grashof number $1 \times 10^{7}$ for $\Delta t=67^{\circ} \mathrm{F}, 24$ which is obtained by trial and error. The overall film coefficlent and required length are calculated by the same procedure as discussed in Chapter VII, with h' derived from Equation (15). The results are shown in Table 22 for the above flow rate. The considerably large requirement of heated length results from the small flow and the insufficient film coefficient on the mud side of the water cooler.

The available 2 " outside pipe is somewhat oversized. Water becomes corrosive to ferrous materials when its surface temperature is high and • 
and dissolved air is present. 22 The use of cooling water at velocities less that $3 \mathrm{ft} / \mathrm{sec}$ is usually avoided and an outlet temperature above $120^{\circ} \mathrm{F}$ is also undesirable. The fouling in low velocity flows becomes a serfous problem and greatly retard the heat transfer. In our case, water velocity is as $10 \mathrm{w}$ as $0.58 \mathrm{ft} / \mathrm{sec}$. (Related fouling problem was discussed in Chapter VIII.) 
CHAPTER XI

\section{TUBE VISCOMETER}

Rheological properties of test fluids are determined by measuring pressure drops across three in-Iine tubes of different diameters based on the following relations.

$$
\tau_{w}=\frac{D \Delta P}{4 L}=K^{\prime}\left(\frac{8 V}{D}\right)^{n^{\prime}}
$$

For the 3 different tubes the following data must be obtained.

1. Density of the fluid at temperature $\rho\left(\mathrm{slug} / \mathrm{in}^{3}\right)$

2. Tube inside diameter $D$ (Inch)

3. Tube length L (inch)

4. Mass flow rate $W$ ( $(\mathrm{l} l u g / \mathrm{hr}$ )

5. Pressure drop $\Delta \mathrm{P}\left(\mathrm{pf} / \mathrm{ft} \mathrm{T}^{2}\right)$

This data is next converted into a $\tau_{w}$ versus $8 \mathrm{~V} / \mathrm{D}$ plot.

$$
\tau_{W}=\frac{D \Delta P}{4 L} \text { and } \frac{8 V}{D}=\frac{32 W}{3600 \pi \rho D^{3}}
$$

The effective viscosity $\left(\mu_{e}\right)$ can now be written as

$$
\mu_{e}=\frac{\tau_{W}}{8 V / D}
$$


The above equations are valid only for laminar flow. Laminar flow will exist in the tube provided the Reynolds No. is less than 2,100 .

$$
\text { Reynolds No. }=\frac{\text { DVP }}{12 \mu_{e}}
$$

To obtain $n$ ! we must vary tube diameter and fix the flow rate or vary flow rate with a single fixed diameter tube. For this project we have chosen the former approach.

The parameter $n^{\prime}$ is obtained from the Rabinowitsch-Mooney equation for steady laminar flow with no slip at the wall.

$$
n^{\prime}=\frac{\log \left(\tau_{w_{1}} / \tau_{w_{2}}\right)}{\log \left\{\left(\frac{8 V}{D_{1}}\right) /\left(\frac{8 V}{D_{2}}\right)\right\}}
$$

Take $n$ ' from the $10 \mathrm{~g}, 10 \mathrm{~g}$ plot. For relatively linear plots of $\tau_{w}$ versus $8 V / D$ the parameter $n '$ can be equated to $n$, the flow behavior index for power law fluids.

The parameter $K^{\prime}$ is now calculated from

$$
K^{\prime}=\tau_{\mathrm{w}} /\left(\frac{8 \mathrm{~V}}{\mathrm{D}}\right)^{\mathrm{n}^{\prime}}
$$

evaluated at $8 \mathrm{~V} / \mathrm{D}=1$

The fluid consistency index for power law fluids is obtained from:

$$
K=K^{\prime}\left(\frac{4 n^{\prime}}{3 n^{\prime}+1}\right)^{n^{\prime}}
$$

Since the above equations are valid only for steady state laminar flows without slipping motions at the walls, there are a few probable errors associated with the tube viscometer and some precautions should be taken: 
1. Some kinetic energy is lost while flowing through expansions or contractions. Tapping the pressure tranducer some distance away from each expansion or contraction will allow the velocity distribution to become fully developed. This "calming" distance will depend somewhat on the entry conditions.

2. When suspensions like drilling fluids flow through a narrow tube, the dispersed phase may tend to migrate. This error becomes more serious when the mud flocculates and the tube bore has scale deposited on the wall. The magnitude of the required correction is of the order of the ratio of particle diameter to the tube diameter. It is usually negligible for particles of clay size, and more significant for larger particle sizes. The relation for its correction is available for Newtonian fluids, but it is difficult to apply to nonNewtonian fluids.

3. The irregularities of tube bore due to the attachments of pressure transducers cause some end effects. The effects are reduced by making the ratios of tube length to radius sufficiently large that the pressure drops along the tube length overwheln those at the ends. The length-radius ratio such as $65: 1$ has been considered safe.

4. The effect of turbulence, which may occur in the situations of low viscosity after heating, heavy mud weight and high pumping speed, should be considered. 
The accuracy of viscometric measurements is determined by the following factors:

1. Precision of the pressure transducers and recording instruments is $\pm 0.85 \%$, or equivalently $\pm 0.09 \mathrm{cp}$ for each $10 \mathrm{cp}$ (0.5\% for the transducers, $0.3 \%$ for the recorder and $0.05 \%$ for the millivolt potentiometer).

2. Tolerance of the tube diameter is \pm 0.002 inch or $\pm 1.5 \%$ for $\frac{1}{2} "$ tubes. The precision of the corresponding viscosity is $0.15 \mathrm{cp}$ for every $10 \mathrm{cp}$.

3. Accuracy of the flow rate measurement is $\pm 0.9 \%$ ideally, based on a 15-second reading using a graduated cylinder.

Total accuracy amounts to $3.3 \%$ approximately, $1 . e ., \pm 0.65 \mathrm{cp}$ for every $20 \mathrm{cp}$. The precision of the temperature measurement by using ironconstantan thermocouples is about $\pm 0.5^{\circ} \mathrm{F}$, if properly pre-calibrated. 
CHAPTER XII

THE SYSTEM

Figure 52, shows the schematic diagram of the planned flow loop. The entire loop contains about $260 \mathrm{ft}$. of $\frac{1}{4} "$ x $0.035 "$ stainless steel tubing. At a circulation rate of $20 \mathrm{gph}$, it takes a fluid volume approximately one minute to complete a cycle of heating, cooling, intensive shearing and returning to the reservoir tank. The heating units are designed based on the circulation rate of $20 \mathrm{gph}$ and higher maximum system temperatures can be achieved by reducing circulation rate. The pressure drop per unit length of the tube varies considerably with mud composition, temperature, flocculation condition and plugging. According to Magcobar's experience with their system, the pressure drop per unit length may range from $0.2 \mathrm{psi} / \mathrm{ft}$ with added thinners being effective to 12 psi/ft in flocculated conditions. 41 Since the vapor pressure of water at $550^{\circ} \mathrm{F}$ is $1050 \mathrm{psia}$, the maximum allowed pressure drop up to the 'hot' section of the $100 \mathrm{p}$ is limited to 950 psi, if the pump pressure is mantained at 2000 psi. That means the average pressure drop is about $4 \mathrm{ps} 1 / \mathrm{ft}$. Higher pump pressure is needed if the flocculation occuring in the 'hot' section causes excessive pressure drop. For further development of the system to achieve $700^{\circ} \mathrm{F}$, the vapor pressure of water unfortunately rises sufficiently in this temperature range (up to $3100 \mathrm{psia)}$ that the required high pressure in the flow loop may become a problem. 
J-IUBE Visconcien

3 WAY VALVE IEST SECTION 1 IEST SECIION 2 IEST SCCTION 3
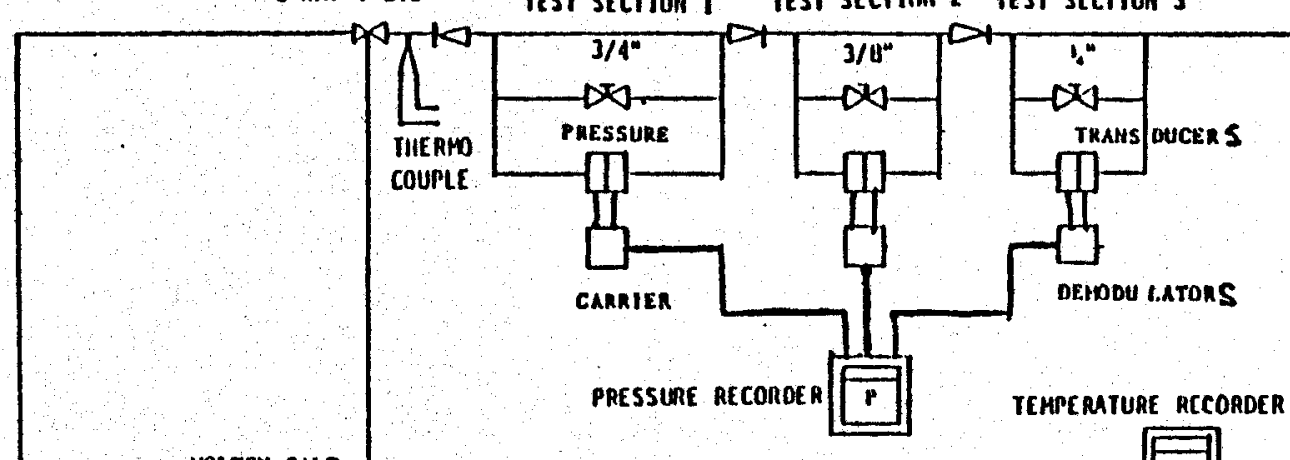

IIEAT EXCIAHGE
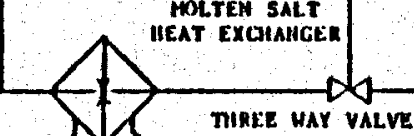

ELECTRICAL HEATINC SECTION

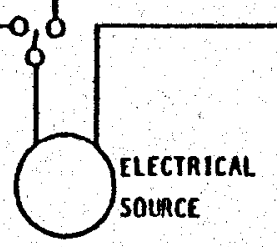

FIGURE 43

DIAGRAM OF FLOW LOOP

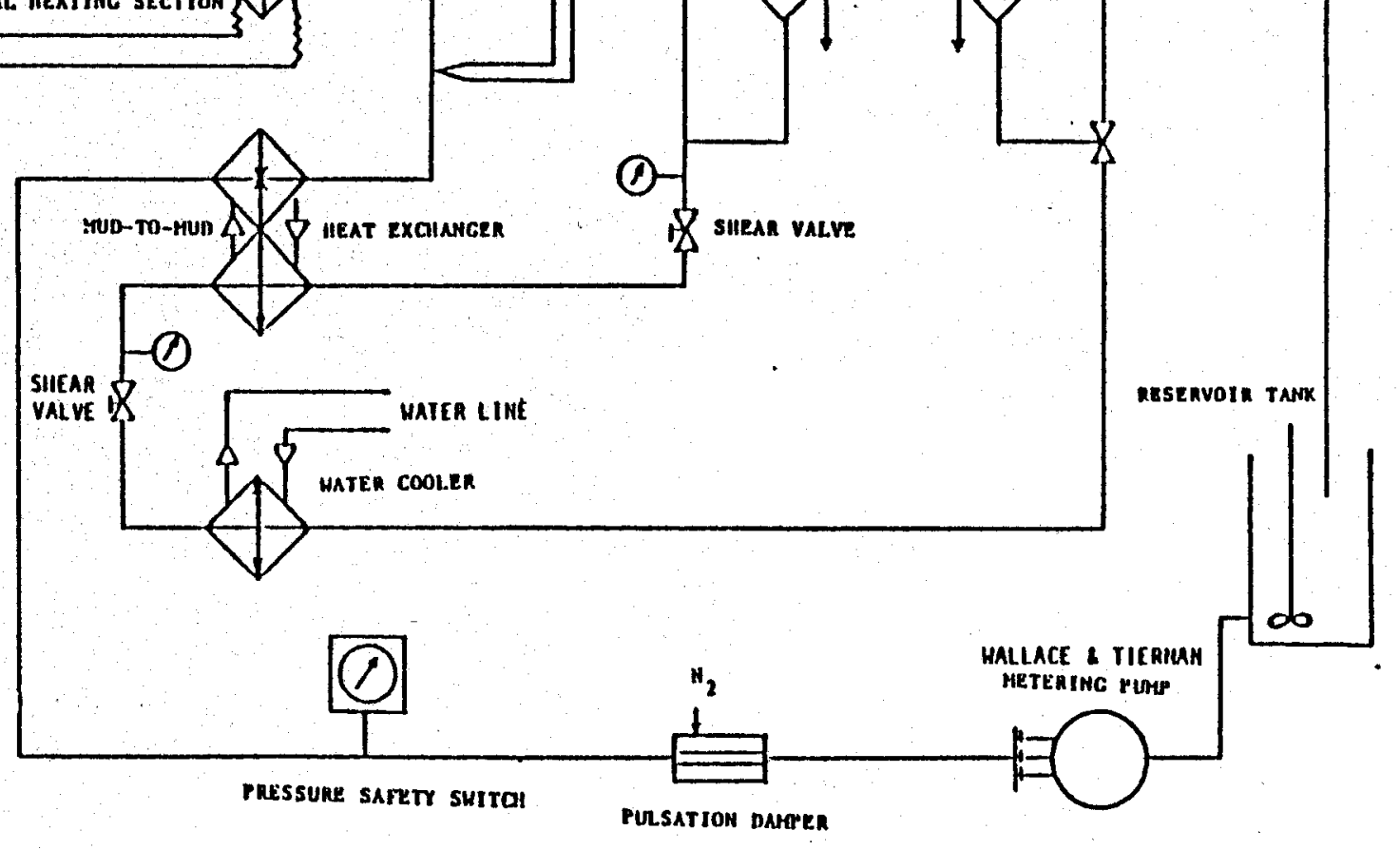


The pressure head in the system is produced by a triplex metering pump with a $\frac{1}{6}$ HP motor, and is malntained by one or more shear valves. A spring-loaded shear valve with tungsten carbide seat and ball can withstand a pressure difference of about 250 psi for a prolonged life in normal cond1tlons 40 (Figure $(-2)$. It wears faster in the cases of high circulation rate and high solidscontent. Ceramic-ball shear valves allow higher pressure differences across them. If the average pressure drop along the tube is $1 \mathrm{psi} / \mathrm{ft}$ or $6 \mathrm{psi} / \mathrm{ft}$, for instance, the pressure on the upstream side of the high temperature shear valve would be about $1840 \mathrm{psi}$ and $1040 \mathrm{psi}$ respectively for pump pressure of $2000 \mathrm{psi}$. Any shear valve in the 'hot' section is not able to keep the test mud from boiling for the latter case, while in the first case it way need three 550 psia-capacity valves totally.

\section{MEASURING AND RECORDING INSTRUMENTS}

Temperature is measured by pre-calibrated iron-constantan thermocouple wires attached to the outer surfaces of tubing. Each.couple is wire wrapped around the tubing tightly and insulated in order to reduce the conduction error. To avoid a temperature gradient close to the measuring function, teflon insulation is provided along the length of lead wire. Thermocouple junctions are positioned on the surfaces of the properly insulated portion of mud tubes to make certain the surface temperature is representative of the fluid temperature.

The electromotive force (emf) of the thermocouples are recorded on a self-balancing "Speedomax" strip recorder. The bililt-in self-compensated reference junction is calibrated by a Leeds \& Northrup 8686 millivolt potentiometer. The spurious emf developed in the thermocouple circuit by 
inductive effects of unshielded ac power leads are diminished by a filter mechanism Inside the recorder. Upon completing the installation of thermocouples and extension leads, each circuit is checked for poor contacts by measuring its overall electrical resistance using an ohmmeter which should show a consistent reading.

The following locations are chosen to attach thermocouples:

1. Between the mud-to-mud exchanger and the electric heating section in order to understand the performance of the former for further development. The efficiency of the Insulation around the electric heating section and the mud-to-mud exchanger can also be determined from this temperature reading.

2. Just before the viscometric test section in order to know the system temperature at which mud properties are measured.

3. Just before the sampling valve in order to control the water flow rate to cool the returning aud to the fixed temperature of $115^{\circ} \mathrm{F}$ at which low temperature properties are measured.

The temperature of the salt bath is to be known hence, a thermocouple well is installed to give an average temperature for the stratifled temperature distribution in the molten salt.

Pressure drops are measured by Validyne variable reluctance transducers.

The feasibility of mud filled circuits for the transducers was examined. A transducer diaphragm of stainless steel 304 was immersed in a bentonfte slurry of $\mathrm{pH} 10$ at different temperatures for one week. No serious corrosion was observed. Hence it was decided to adopt mud filled circuits in 
which the transducer diaphragms are cleaned frequently such as once a week.

A carrier demodulator connecting the transducer and recorder converts inductance ratio of the two coils in each transducer to dc output voltage which is in turn picked up by the Speedomax recorder. Transducer indicators with built-in carrier demodulators can also be used for this purpose. Callbration is made by a portable millivolt potentiometer. According to the maximum possible pressure drop per unit length as mentioned before, the operating range of the transducers can be selected as 50 psia.

\section{OTHER TEST APPARATUS}

The properties of dynamic filtration and corrosivity of test fluids are measured at high and low temperatures by the in-Iine apparatus as shown in Figures 53 and 55 . The dynamic fluid loss tube consists of a 18"-long, porous, sintered, stainless steel tube which is jacketed by a stainless steel casing. A differential pressure of 100 psi across the filter tube is usually maintained by regulating nitrogen pressure in the annulus. The amount of filtrate collected in the annulus is measured intermittently through a sampling valve.

Corrosivities of test fluids are determined by inserting sample coupons in the holder slots milled along the Inside pipe wall (Figure 55). Welght losses of the coupons are examined to study the corrosion conditions at different temperatures. 
CEAPTER XIII

TEST CONTROLS

As mentioned before, both temperature and time influence the rheological and filtration properties of drilling fluids. Except for the fully aged muds, the mud propertles change with time at a certain temperature level. Proper interpretation of experimental data calls for wellcontrolled test procedures:

I. Preliminary tests:

(1) Calibrate the temperature recorder with a millovolt potentiometer, and calibrate the pressure recorder and transducer Indicators or carrier demodulators by a O-tube manometer.

(2) Check the pressure pulsation of the pump discharge and circulation rate to decide the necessity of a pulsation damper or a flow control valve in the flow loop.

(3) Investigate the probable plugging problem by placing a fine wire longitudinaliy on the inside tube wall close to the electric heater. Correlate the pressure drop of the mud across the electrical heating section versus the chosen wattage which can be changed with the wattmeter. Check the scale on the wire which is pulled out after heating with different heat flux densities. Define the maximum operating temperatures of the section for different wattage 
densitits based on the accumulation of scale on the wire or the increased pressure drop across the section.

\section{Experimental testing:}

The testing procedures depend on whether temperature or time effects associated with the aging problem is of concerm. The temperature and chemical treatments should be controlled properly to yield interpretable data:

(1) Investigation of the time required to stabilize mud samples: Constant temperatures need to be maintained. It is advantageous to use strip recorders for acquisition of temperature and pressure data. Integrated information from the high and low temperature test sections will reveal a better picture of the properties during heating and after heating. Since apparent viscosities of drilling fluids depend on shear rate, it is preferable to maintain a constant circulation rate throughout each run with a control valve if the flow rate pulsates excessively. To study temperature effects on the aging time, the same procedure is repeated but at higher temperature levels until flocculation temperature or the system capacity is reached. "Hysteresis" effect could be checked by decreasing the cemperature levels from the above maximum achleved temperature. If there is a hystersis effect present, it means the mud stabilization depends on its thermal history as does the shearing history of thixotropic materials. It is important that no new chemicals are added to the testing fluid during operation.

(2) Investigation of temperature effect with negligible time . 
influence: By adjusting the wattmeter properly, the mud temperature can be increased smoothly, as displayed on the temperature strip recorder. The rate of Increase of temperature is preferably comparable to the circulation cycle of about two minutes, so that the time factor can be ignored The resulting data can be compared to those obtained by means of batch testing such as Hiller's high temperature rotational viscometer, 17 in which no time factor is involved. If fully aged muds are tested by this method, the experimental data should be reproducible. When insufficiently aged muds are tested at different times, the results will indicate the trends of thermal stabilizing, although the routes approaching stabilized conditions are by no means unique but dependent on the previous aging history. 
FIGURE 44

FIITRATION TEST UNIT

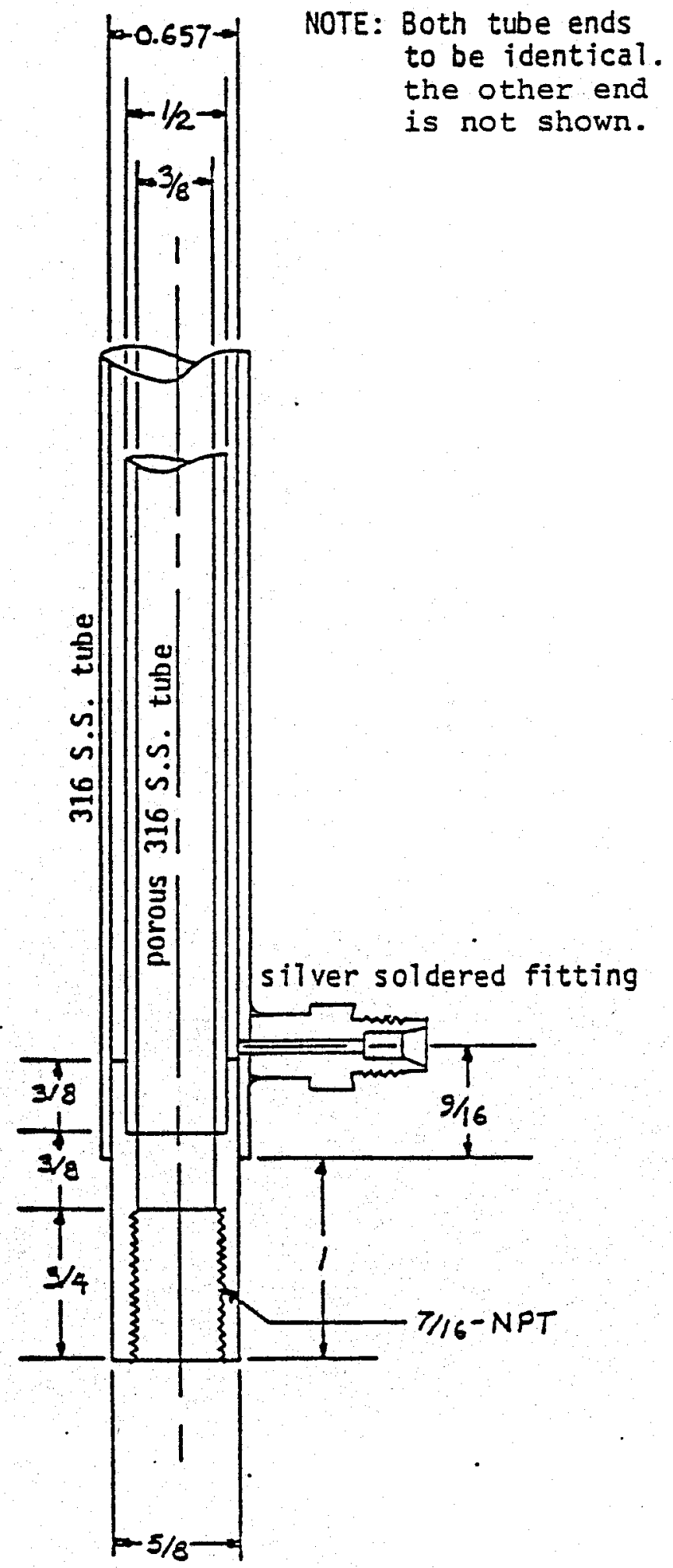


FIGURE 45

SHEAR VALVE

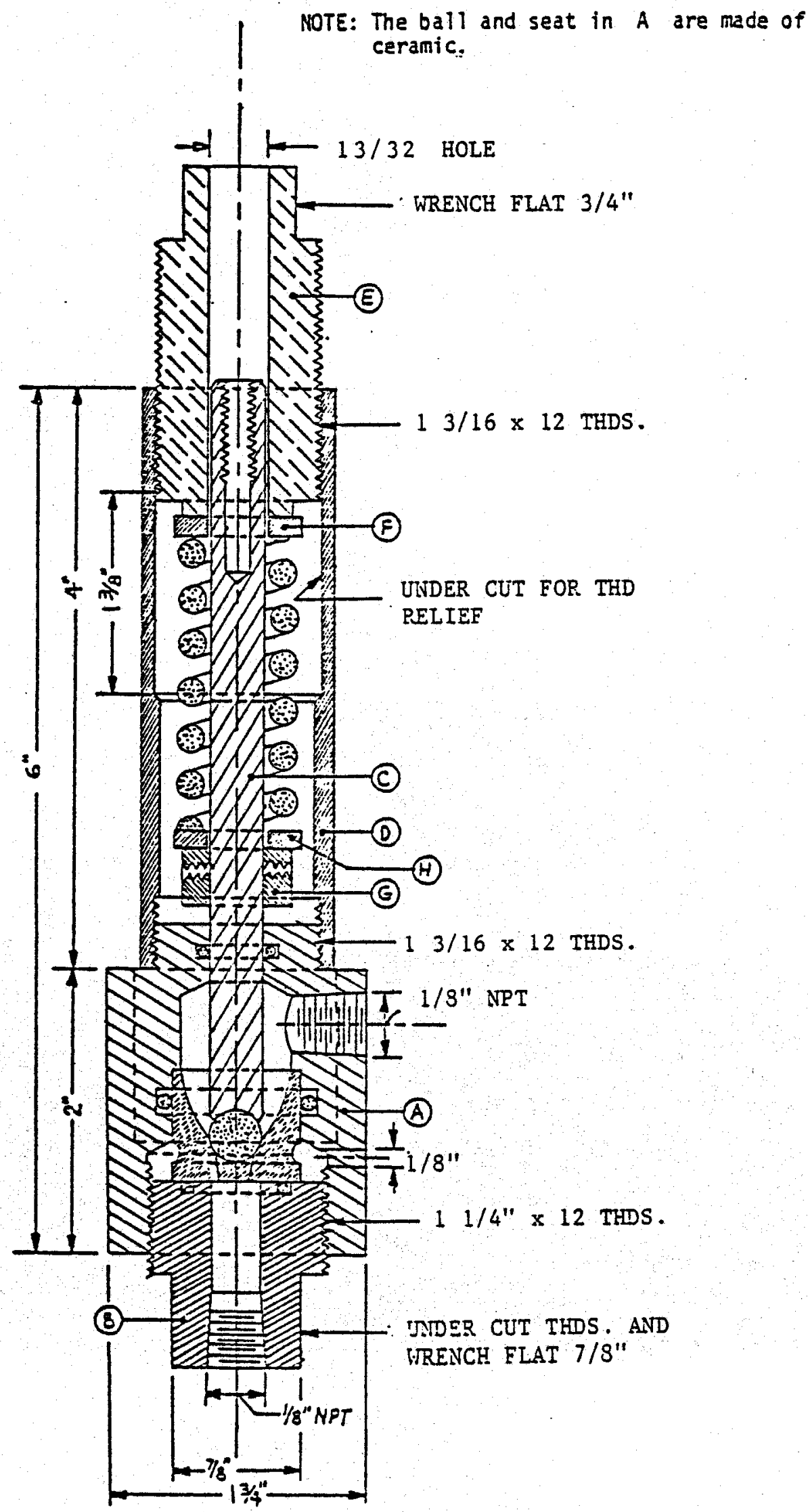


FIGURE 46

CORnOSION COUTON HOLDER
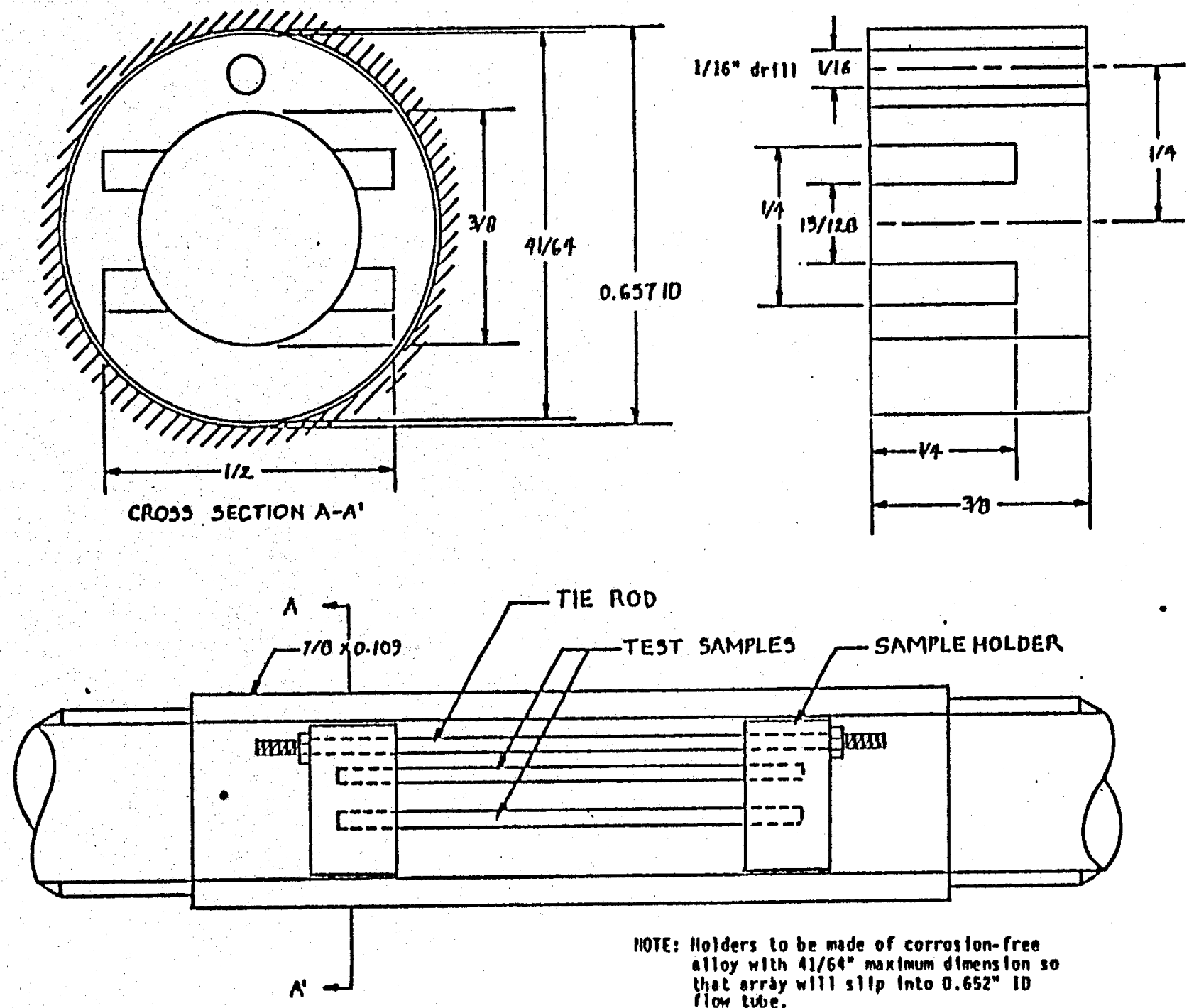


\section{NOMENCLATURE}
$A, A^{\prime} \quad$ Eeat transfer area, $f t^{2}$
B
Coefficient of thermal expansion, ${ }^{O} F^{-1}$
Cp Heat capacity, BTU/LB ${ }^{\circ} \mathrm{F}$
d Tube diameter, ft.
8 Gravitational acceleration, $32.174 \mathrm{ft} / \mathrm{sec}^{2}$
$G$ Mass flow rate per unit area, $L B / h \mathrm{ft}^{2}$
Gr Grashof number $g \beta \rho^{2} \dot{d}^{3} \Delta t / \mu^{2}$, dimensionless
Gz Graetz number $W C_{p} / k$ L, dimensionless
$h, h^{\prime} \quad$ Fill coefficient, BTU/h $\mathrm{Et}^{2}{ }^{\circ} \mathrm{F}$
EP Motor horsepower
$k$ Themal conductivity, BTU/h $\mathrm{ft}^{2}{ }^{\circ} \mathrm{F} / \mathrm{Et}$
$K$ Fluid consistency index of power law model pf $\sec ^{n} \mathrm{ft}^{-2}$
$K^{\prime} \quad$ Power law flow parameter, pf $\sec ^{n^{\prime}} \mathrm{ft}^{-2}$
$I, L_{1}$ Heat transfer length of tube or length between taps of pressure transducers, ft.
1 Length of the outside cylinder of the molten salt heat exchanger, ft
$n$, $a^{\prime}$ Flow behavior index of power law model, dimensionless
Nu Nusselt number $\mathrm{h} d / \mathrm{k}$
p Fressure drop, psi
Pr Prandtl number $C_{p} \mu / k$, dimensionless
PV Plastic viscosity of Bingham plastic model, $c p$
Q Volume flow rate, $\mathrm{ml} / \mathrm{sec}$ 
Subscripts or Superscripts

1

2

,

b

e

i

$\circ$

w

Refer to inlet condition

Refer to outlet condition

Prime: refer to shell slde of heat transfer

Refer to fluid bulk

Refer to equivalent diameter

Refer to inside surface, or insulation

Refer to outside surface

Refer to wall 


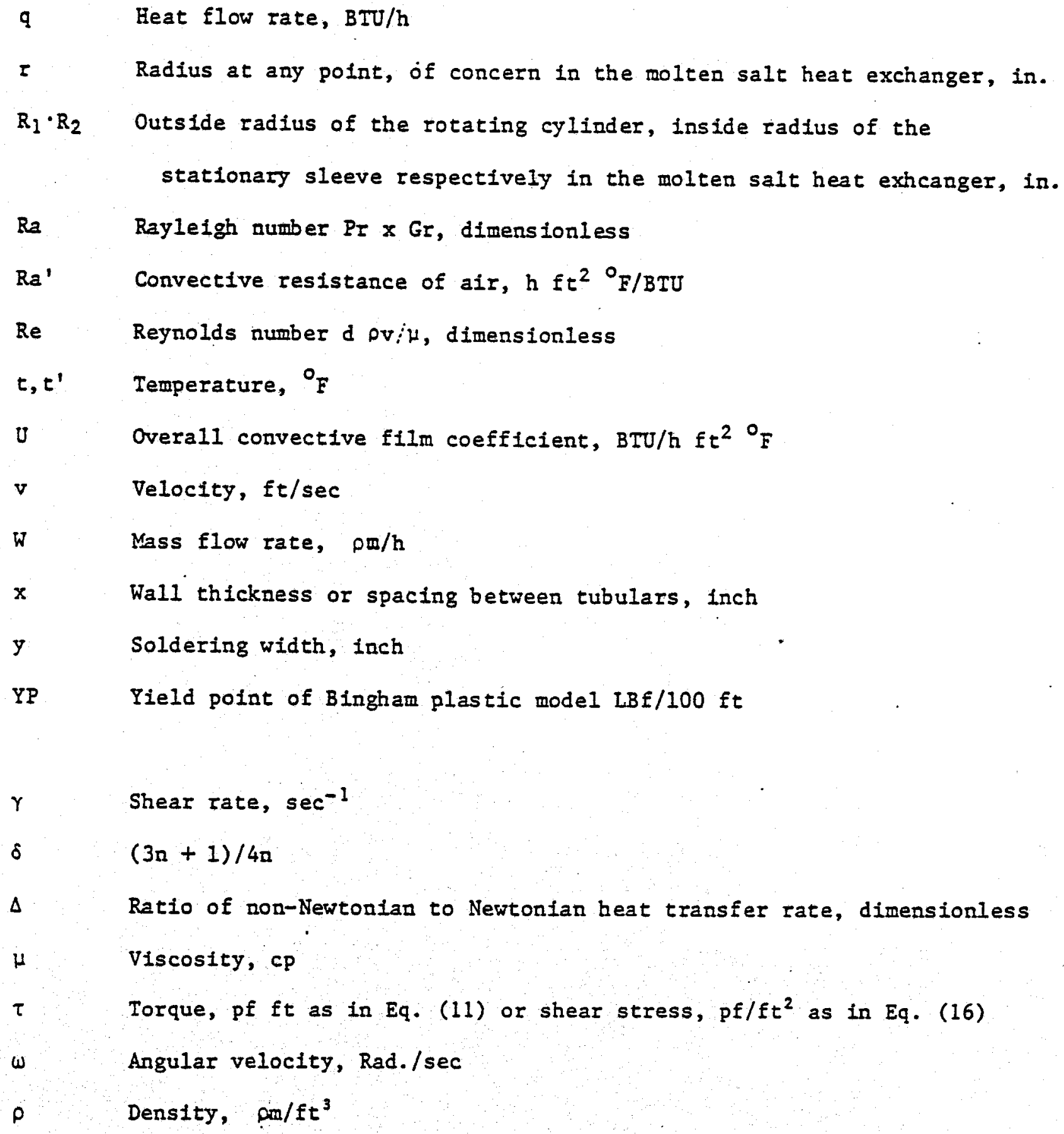


APPENDIX B 


\section{INTRODUCTION}

The need for chemical process applications requiring temperatures above $450^{\circ} \mathrm{F}$ has speeded deyelopment of new chemicals for such use. The development of Dow-therm A in 1932 for heating applications raised the operating temperature level to $700^{\circ} \mathrm{F}$. Then HTS (heat transfer salts) was offered in 1937 for temperatures above $700^{\circ} \mathrm{F}$. Presently Hitec is available with melting point of $288^{\circ} \mathrm{F}$, and it is suitable for use up to $1,000^{\circ} \mathrm{F}$.

\section{TECHNICAL APPROACH}

The molten salt apparatus consists of the following:

1. Heat exchanget shell,

2. Drilling fluid tubes,

3.- Drain system

4. Heating elements and temperature controllers

\section{Heat Exchanger She11:}

As shown in Figure 56 , the heat exchanger shell consists of welded flanged sections to form a cylindrical shell with three side flanges and flanges at bottom and top.

Construction materials:

Sections are carbon steel which has proven to be entirely satisfactory, from both the corrosion and the strength points of view at elevated temperatures. 


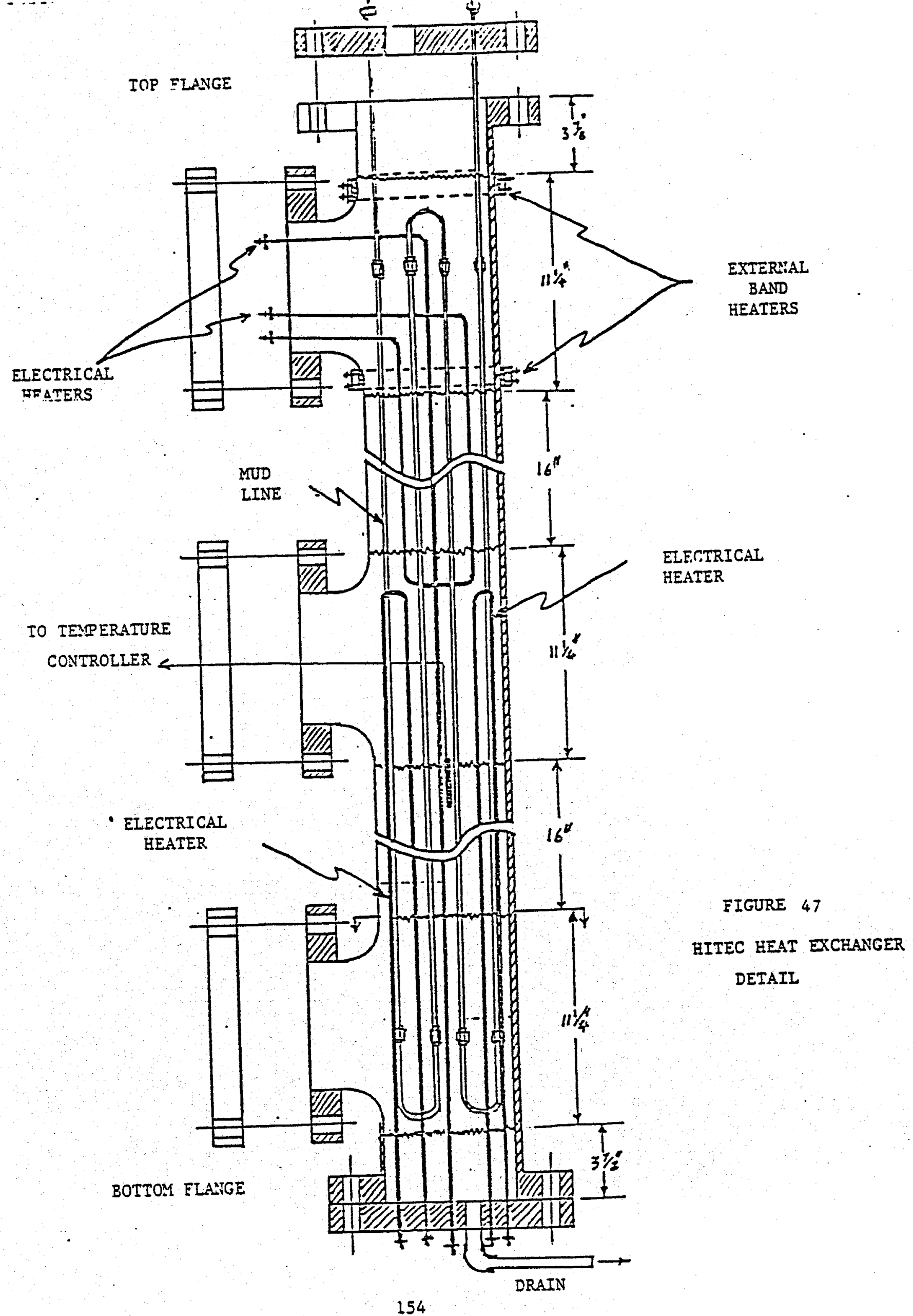


The flanges are circular flat machined, 12" diameter, 1.3" thickness with 12 bolt holes. The gasket is a brass asbestos 0 ring, 6" ID, 1/8" thickness. Bolting process should be made when the shell 1s hot. The top flange has two $1 / 4^{\prime \prime}$ holes for output and input fluid tubes and one inch diameter hole for the 1" pipe, used as open pipe relief in case of tube leakage. The first side flange $(s-1)$, has 3 $0.315^{\prime \prime}$ holes for the 2 heaters. The second side flange (S-2), has only one $1 / 2$ " hole for the temperature controller $3 / 8$ " OD mercury bulb which is connected to the controller by a $1 / 8^{\prime \prime}$ stainless steel capillary. The bulb and the capillary fit inside the molten salt heat exchanger shell. The bottom flange $(S-3)$, has $5-0.475^{\prime \prime}$ holes threaded about $3 / 8$ " depth in the flange to fasten heaters. A $1 / 2^{\prime \prime}$ drain pipe comes from the bottom to the drain tank.

\section{Drilling Fluid Tubes:}

About 30 feet of seamless stainless steel 1/4" OD tubes are used. The tube system consists of six lines connected in series configured on a cylindrical curface inside the shell, as shown in Figure 56 , flaring tube type of fittings are used. Output and input lines are connected with loop through two-way valves. The tubes were static tested to 12,000 psi pressure without leaks.

\section{Drain Systems:}

The drain system consists of:

a. Drain tank of size $27^{\prime \prime} \times 12^{\prime \prime} \times 12^{\prime \prime}$ made from $1 / 16^{\prime \prime}$ steel designed to be able to fix 4 strip heaters on three sides 
of the tank to keep it heated to about $300^{\circ} \mathrm{F}$ just above melting point of the salt.

b. Drain tank is connected also with two pipe lines. The first, comes from the heat exchanger shell in a $L$ shape and it is heated with 3 heaters routed around the one inch pipe to keep the pipe heated above $300^{\circ} \mathrm{F}$. This prevents salt plugging. The second is a horizontal $1 / 2$ " pipe to act as a drain from the heat exchanger to the tank. A valve is placed in the line. The tank has a cover with four $3 / 8^{\prime \prime}$ bolts to keep it sealed when the test is running.

\section{Heating Elements:}

a. Inside the Shell:

Two heaters, UTU type, $3 / 8$ " cross section, high watt density (40-9/16" total length, 38-5/16" heated length, 240 v,

3.6 KW). Tubular heater - triangular cross section. One heater, TRI $0.315^{\prime \prime}$ dia. Incoloy sheath, round cross section. (78", 71-1/4", 240 v. 2350 watt). One heater, TRI type $0.246^{\prime \prime}$ dia., Incoloy sheath, (52", 47-1/2", 240 Volt, 1045 Watt).

b. Outside the Shell:

Two band type heaters at the top of the shell to maintain the temperature distribution uniform at the top and bottom of the salt. (Type HB 1-1/2" wide, 7" barrel diameter, 1000 watt, 240 Volt). 
c. The Drain System:

Drain Tank:

Two strip heaters, Type PT $1 \frac{1}{2} "$ wide, two bolt teminals at right angles, $* 25 \frac{1}{2} "$ long, $240 \mathrm{v}, 750$ Watts). One

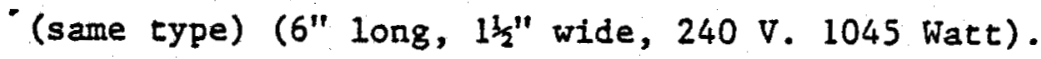

Connected drain pipes:

Two tubular heaters, round cross section, type TRI

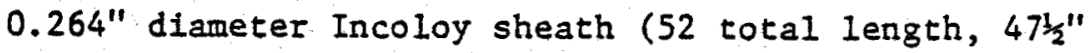
heated length, $240 \mathrm{~V} .1045$ Watt). One (same type) (94", $89 \frac{1}{2} ", 240$ V. 1965 Watts).

\section{Temperature Controllers}

Two temperature controllers have been used to monitor and adjust the temperature in molten salt and drain tank. (MF2 type - ranges to $1,000^{\circ} \mathrm{F}$ indicating). Mercury filled bulb and capillary can be flush mounted or wall-mounted. (208 Volt, 2 amps, 2.7" bulb length). Both of them are connected with 30 amps contactor relay to be able to give the power required for the system.

\section{Properties of Hitec:}

The common composition of Hitec is $55 \%$ sodium nitrate, $45 \%$ sodium nitrite.

a. The freezing point of a dry mixture of aTS is approximately $288^{\circ} \mathrm{F}$. However, the salt is hygroscopic when granular. The absorbed molsture reduces the melting point. The M.P. rises when the same salt (sample) is used more than one time. 
b. Heat Capacity. The heat capacity emphasizes the excellent thermal capacity of Hitec on a volume basis of $0.73 \mathrm{cal} . /$ (cc.) $\left({ }^{\circ} \mathrm{c}\right)$ at $300^{\circ} \mathrm{F}$, and $0.62 \mathrm{cal} /(\mathrm{cc})\left({ }^{\circ} \mathrm{c}\right)$ at $1,000^{\circ} \mathrm{F}$.

c. Viscosity. Viscosity is important to determine the pumping capacity for circulated molten salt. Hitec has a relatively low viscosity of $8 \mathrm{cp}$ at $400^{\circ} \mathrm{F}$ and $2 \mathrm{cp}$ at $1,000^{\circ} \mathrm{F}$.

d. Thermal Conductivity. Thermal conductivity values for Hitec from several sources ranges from a high of 0.66 reported by Gambill ${ }^{12}$ to a low in the range in 0.17 to 0.24 by Nelmark vargaftik et al. ${ }^{41}$. It is recommended that the value of 0.35 be used because it successfully correlates with other heat-transfer data.

e. Chemical Stability. Hitec is chemically very stable to $850^{\circ} \mathrm{F}$ from 850 to $1,100^{\circ} \mathrm{F}$ the nitrate in the mixture undergoes a slow thermal decomposition to $\mathrm{NaNO}_{3}$ and $\mathrm{Na}_{2} \mathrm{O}$ with evolution of $\mathrm{N}_{2}$. As the temperature rises, the rate of reaction increases so that at $1,500^{\circ} \mathrm{F}$ nitrogen evolution is so rapid that the salt mass appears to be boiling. When Hitec is in contact with air above $850^{\circ} \mathrm{F}$ three additional types of reactions take place to a limited extent. The principal one is the oxidation of the nitrite to nitrate. The other lesser reactions are the absorption of $\mathrm{CO}_{2}$ to form carbonates and of water vapor to produce hydroxides.

\section{Insulation:}

The insulation material (fiber-glass $\frac{1}{2}$ cylindical shell pieces 
are used to cover the heat exchanger shell, piping and the drain tank. 8. Performance of the Hitec System:

During the period of this contract it was not possible to run the Hitec system to full temperature. This was due to repeated failure of the inside shell electrical heaters. This falure was due to a manufacturing defect.

In early tests an electrically resistive type cement was used to seal the heater elements and tubing to the end flanges. This proved to be unsatisfactory in that the cement permitted seapage of water and dissolved Hitec out of the system. To provide an absolute seal against leakage, special seals were fabricated in the University of Oklahoma machine shop. This seal has performed well and no further problems have been encountered with leakage. 
APPENDIX C 


\section{ELECTRICAL CIRCUITRY FOR THREE TUBE VISCOMETER}

\section{Electrical Circuitry:}

The electrical aspect of the flow loop consists of two main divisions. The first is power distribution and control; this is illustrated in Figure 57 . The second division is electrical measurements of the flow loop dynamic variables; these are temperatures and pressures and are illustrated in Figures $58 \mathrm{~A}$ and $58 \mathrm{~B}$.

Referring to Figure 57,59 , and 60 , it can be seen that the power control and distribution system provides for the manual and automatic aspects of loop control. Manual operations encompass the three main power systems of the flow loop that is, pump power, test section heaters, and salt section heaters. Manual, On-off, control of these functions is obtained through the use of push button-latching relaycontactor arrangements. The system has the advantage that it is "fail safe"; it will not reapply power to the $100 \mathrm{p}$ if the main power is interrupted. The system also readily adapts to the inclusion of a "panic button" to shut down the loop in case of an emergency. Panic button operation is implemented in this system both as a manual push button and as a pressure limit switch.

Three channels of temperature control are provided in the flow loop test section. Each channel has its own thermocouple and temperature 
controller. Power switching to the test section heaters is done using contactors. Temperature control in the salt section is accomplished using liquid filled probes and electromechanical controllers operating contactors.

The pressure recording system for the flow loop is shown in Figure 58A. A total of seven transducers measure loop pressure at five points. The quartz transducers and charge amplifiers are Kistler A while the remaining transducers and readouts are Validyne $B$. The Validyne transducers are of the varlable reluctance type. The output signals from the pressure readout units are channeled through gain control amplifiers and connected to a multichannel strip chart recorder. The gain control amplifiers allow recorder calibration in engineering units,

The temperature recording system for the flow loop is shown in Figure 58B. This system consists simply of iron-constantan thermocouples connected to a multichannel strip chart recorder equipped with thermocouple amplifiers.

A. Mode1 5001 charge amplifier and model 6005 quartz transducer

B. Model DP15 differential pressure transducers and models CD12 and CD223-A demodulators and readouts.

C. The strip chart recorders are Leeds and Northrup 12 channel Speedo-Max recorders.

\section{Electronic Filtering of Pressure Measurements of the Flow Loop:}

During operation it was found that interpretation of pressure data was abscured by high frequency (about $1 \mathrm{~Hz}$ and greater) noise arising from pump pressure pulses and random sources. This noise was especially 
bothersome when attempting to obtain real time data from the digital pressure readouts. As an aid to data reduction and interpretation low pass filters were installed in all of the Validyne equipment. The filter circuit is shown in Figure 61. The filter can be switched in and out of the circuit so its effect on the pressure data can be interpreted in real time. It was determined experimentally that a $1 / 3 \mathrm{~Hz}$ filter adequately suppressed the noise without degrading the desired data.

It was found that the Kistler piezoelectric transducers were unsuitable for the type of pressure measurement needed in the flow loop. The problem arises because the piezoelectric transducer responds only to changes in pressure. Thus the Kistler transducer would register the initial pressure change when first starting the pump but electrical leakage in the charge amplifier drains off the initial charge induced in the transducer by the application of pressure. Thus the output of the transducer slowly decreases until the readout only indicates transient pressure fluctuations. The performance of the Validyne variable reluctance transducers was found to be satisfactory when using the previously described filters. One disadvantage of the Validyne transducers is that they cannot function at temperature exceeding $170^{\circ} \mathrm{F}$ and must be isolated from the hot flow loop with long capillaries.

Figure 62 shows the circuit diagram for the five channel gain control ampliflers. These were needed for signal conditioning and calibration since these functions were not provided in the speedo-Max recorder. 
FIGURE $48^{\circ}$

POWER CONTROL AND DISTRIBUTION BLOCK

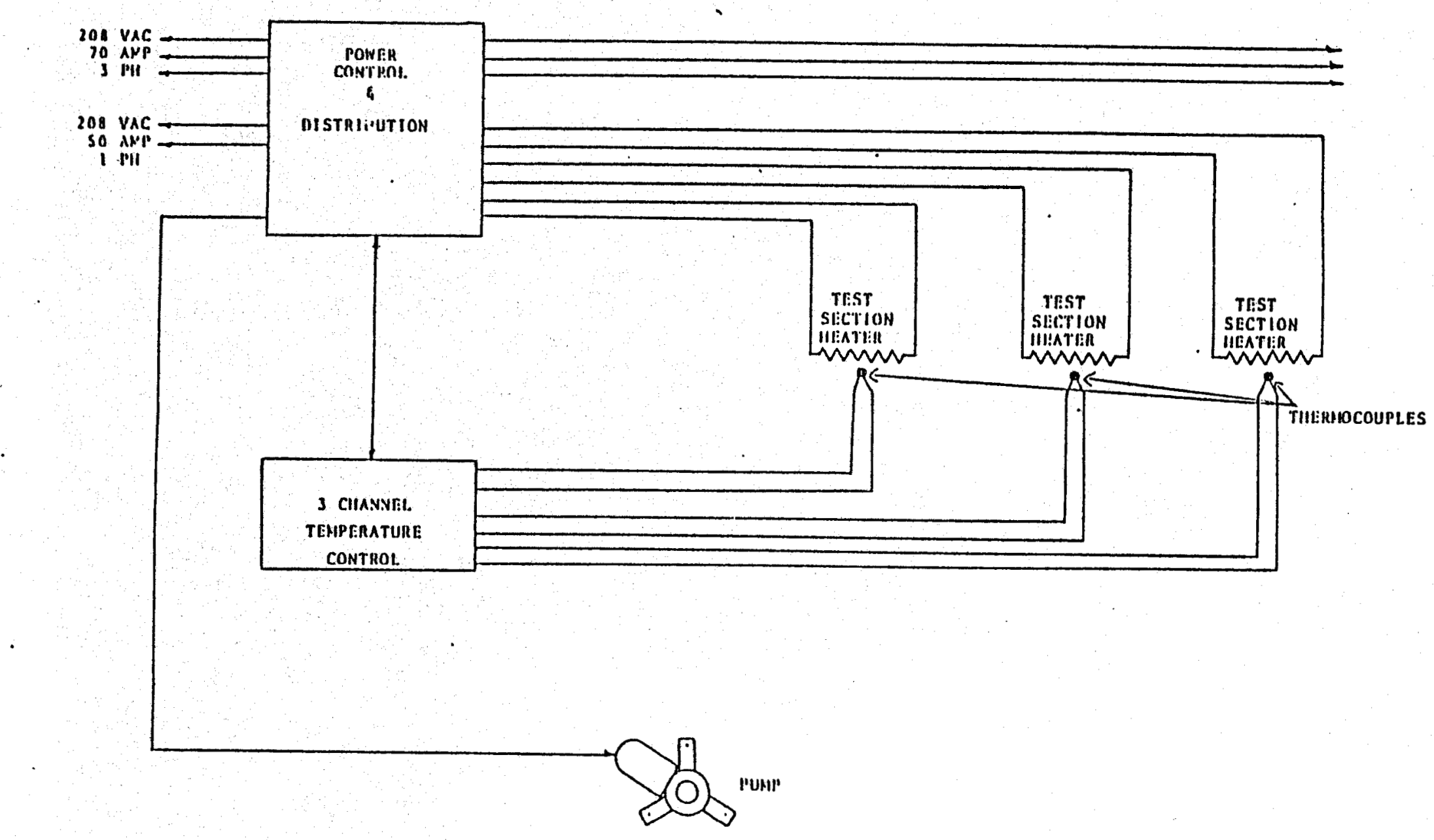




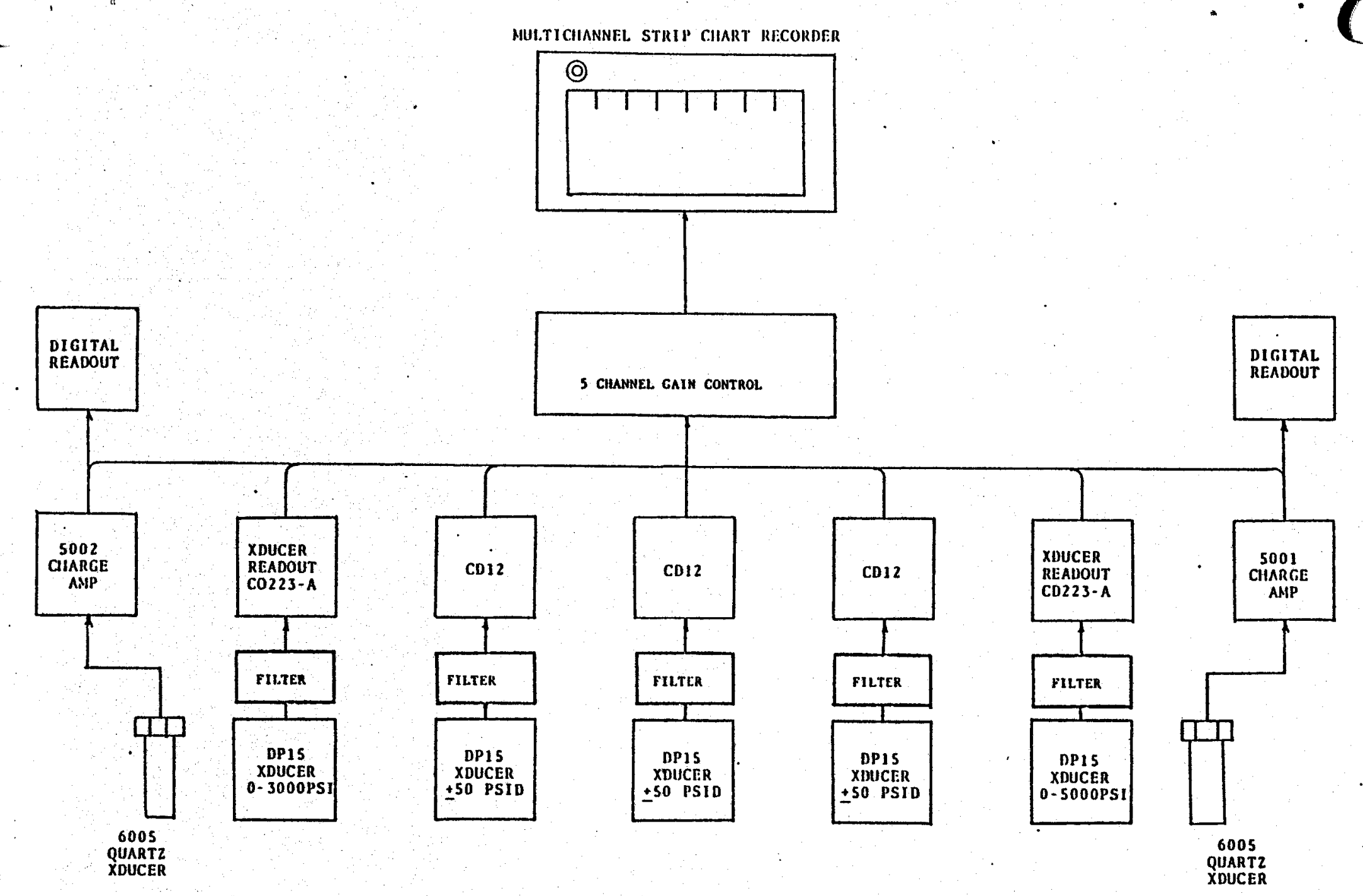

FIGURE 49

PRESSURE RECORDING SYSTEM 
MULTICHANNEL STRIP CHART RECORDER

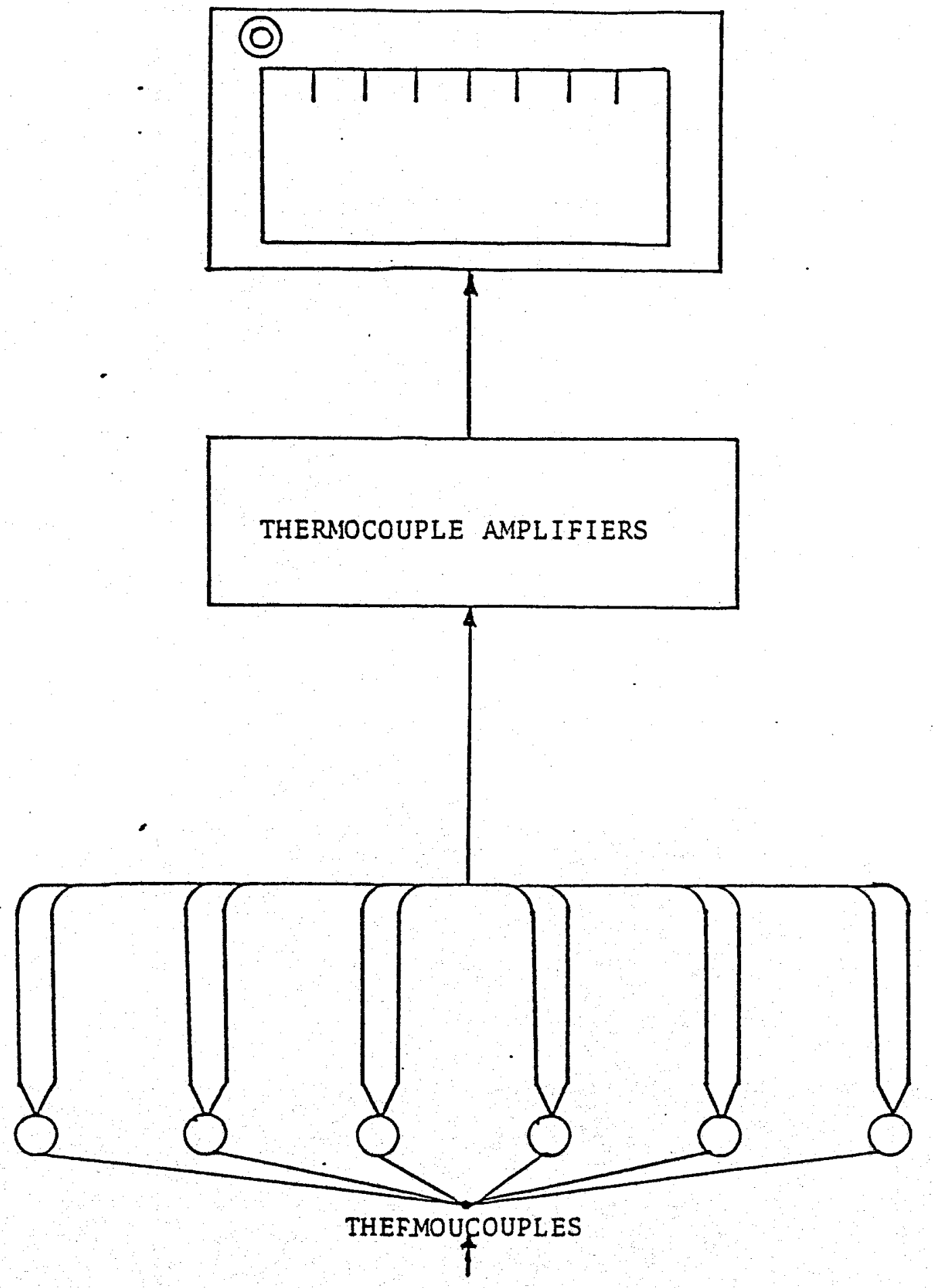

FIGURE 50

TEMPERATURE RECORDING SYSTEM

166 
FIGURE 51

MAIN POWER SWITCHING

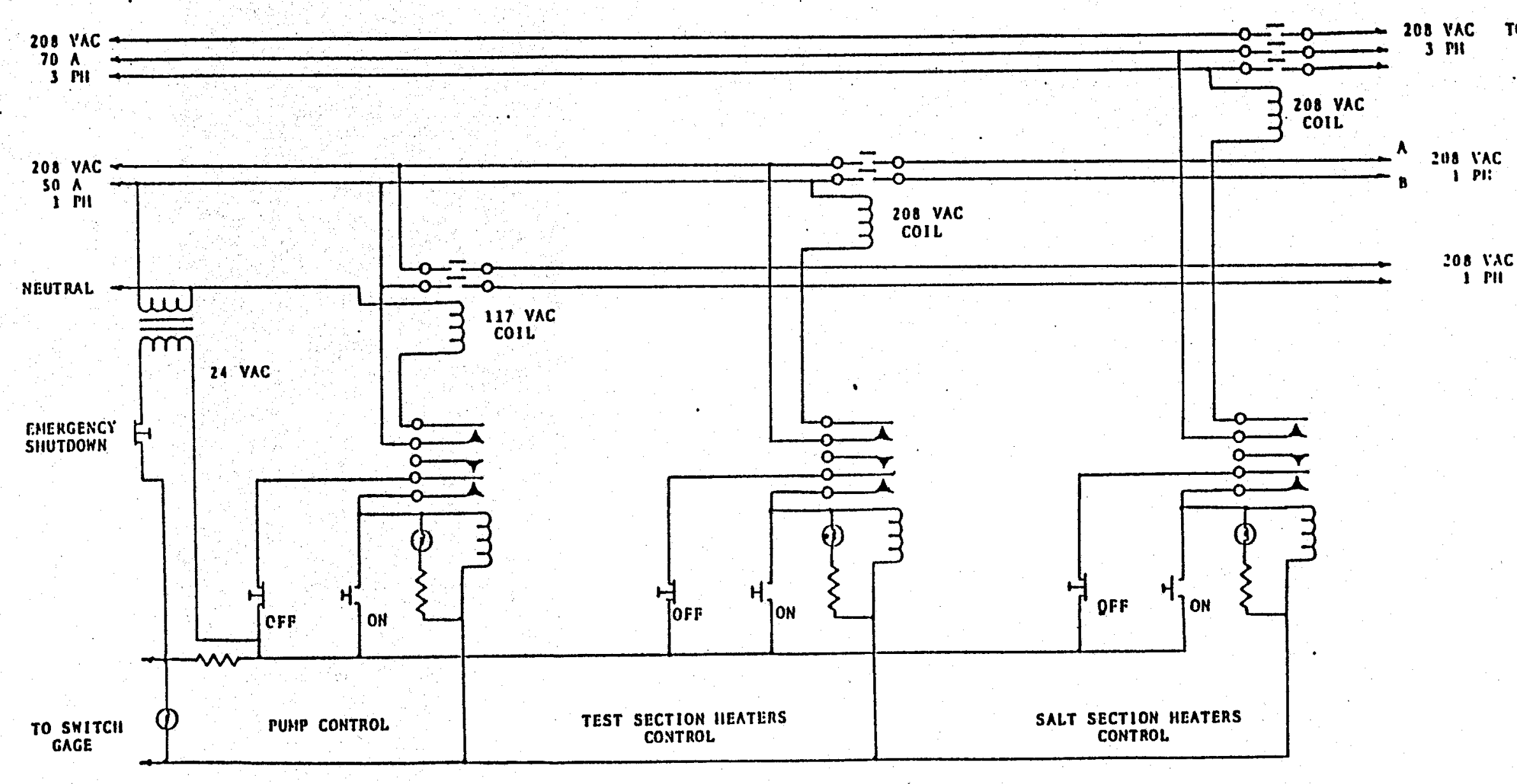

IO SALT SICTION MEATrRS 


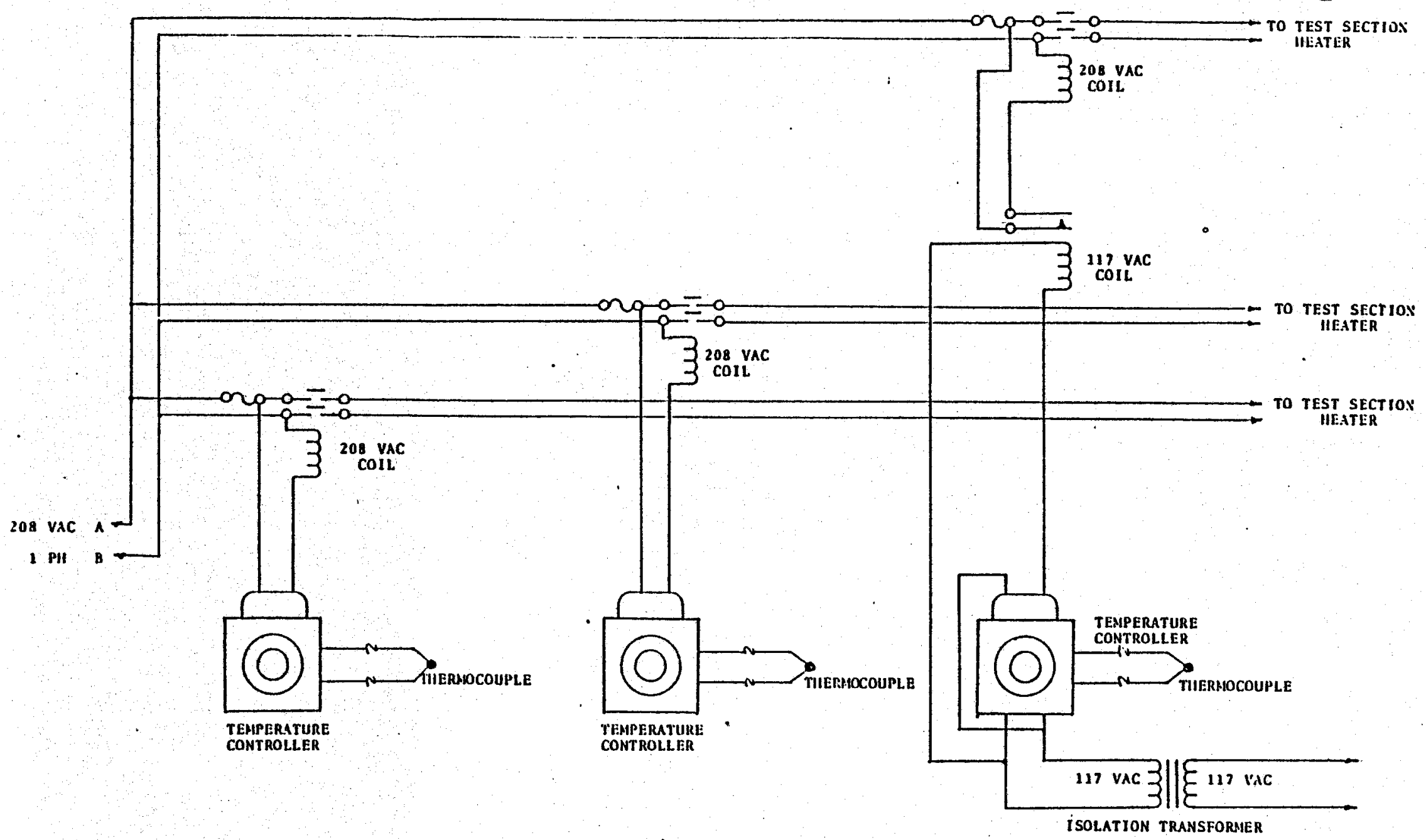

FIGURE 52

TEST SECTION HEATER POWER DISTRIBUTION AND CONTROL 


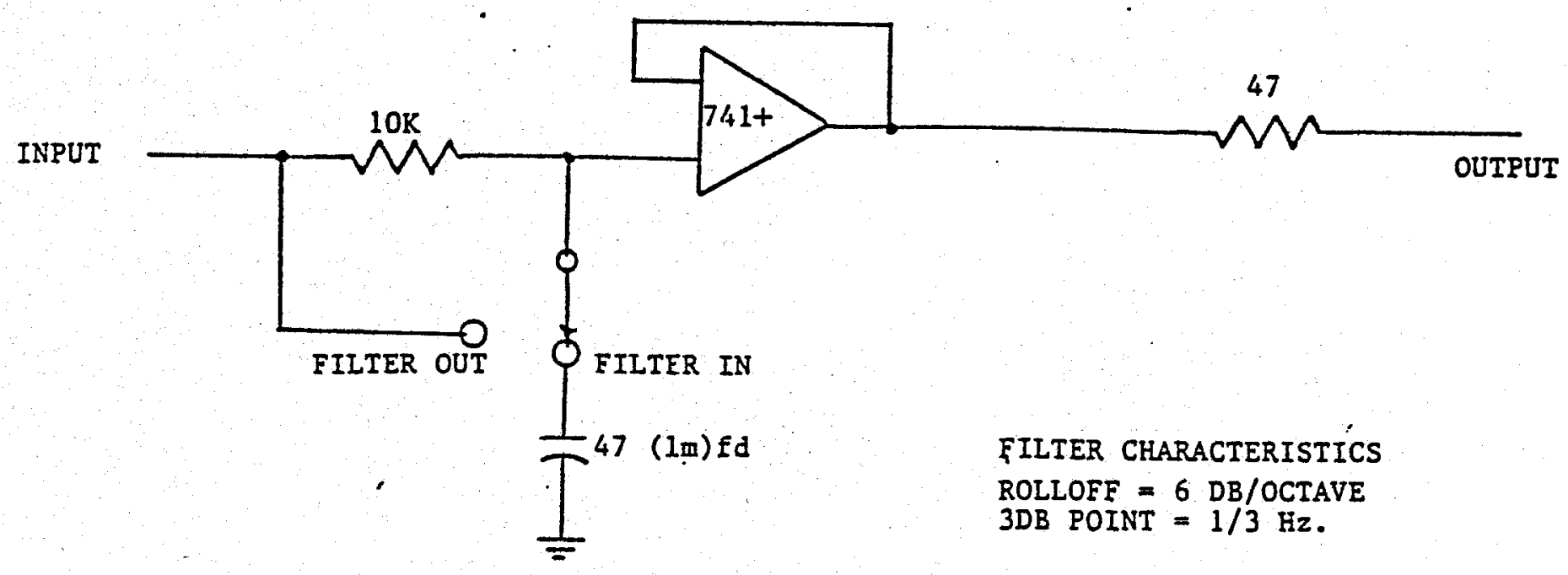

FIGURE 53

1/3 $\mathrm{Hz}$ FIITER 

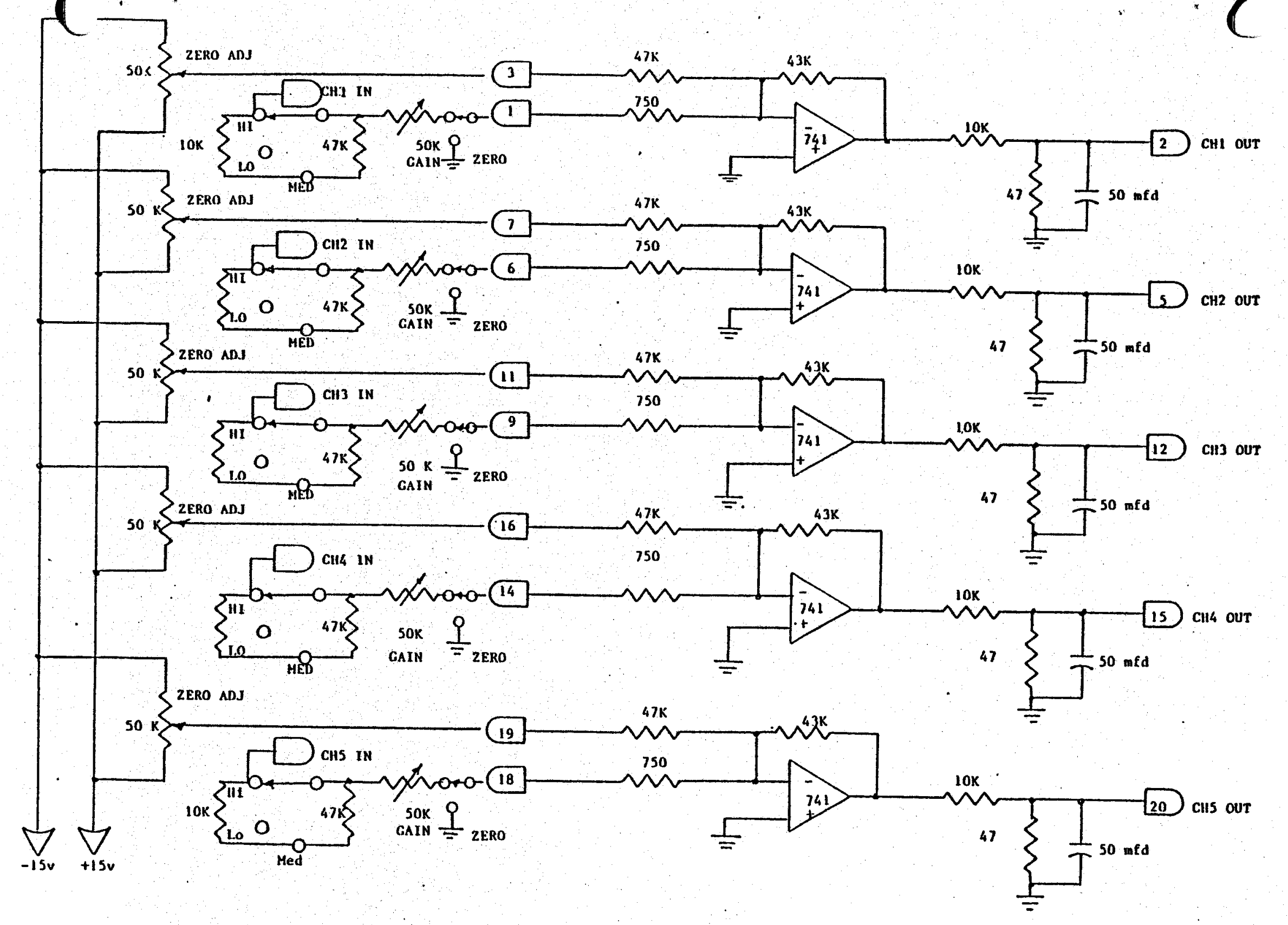

FIGURE 54

FIVE CHANNEL GAIN CONTROL

NOTE: GAIN - A

HI $\quad .85 \leq A \leq 57$ MED . $71 \leq \hat{a} \leq \frac{1}{\leq} 4$ 
APPENDIX D 


\section{FILTRATE TESTS}

A system to study dynamic filtration was included in the flow 100p. The major features of the system are discussed in Appendix A. The results of specific tests are discussed here.

A. Determination of filtrate loss of a clay-water slurry containing 6.PPB chrome lignosulfonate.

The test utilized a dynamic filtration cell designed by Reese Wyant of Dresser Magcobar. The test cell is capable of operating at $550^{\circ} \mathrm{F}$ and 3,000 psi. A diagram of the filtrate cell is included as Figure 63 and a diagram of the flow loop network pertinent to the operation of the filtrate cell is included in Figure 64.

The test procedure, results and conclusions are presented for the chrome lignosulfonate slurry, the test temperature was $450^{\circ} \mathrm{F}$ and system pressure is maintained above 1,500 psi whenever possible

\section{Test Procedure}

The test procedure depends on the stability of the fluid being tested. The fluld should be tested in laminar flow and under steady state conditions. The fluid should have a constant temperature and the pressure fluctuations should be kept to a minimum.

Once the fluld has attained a stable condition the test may be initiated. The time of test begins immediately after the fluid is allowed 
to flow through the filtrate cell. Valves $2,3,4,5,6$ (Figure 64) are initially closed and valve one is open. When the test begins valve 1 is left open until valves 2 and 3 are opened.

At certain time intervals, 5 minutes, the collected filtrate is drawn from the filtrate cell by opening valve 5. The tubing leading from valve 5 is looped in an Ice bath to cool the fluid. The nitrogen input pressure is then adfusted to approximately 500 psi above the flowing pressure. Valve 4 and Valve 6 are opened gradually allowing the filtrate to collect in a graduated cylinder. The volume accumulated is recorded as well as the time the sample was taken. This procedure is continued until termination.

\section{RESULTS}

The results of the filtrate test on the chrome lignosulfonate fluid are included in Table 27 and Figure 65 . The test was terminated due to excessive pressure fluctuations resulting from flocculation of the test sample.

CONCLUSIONS

Data indicates that the filter cake thickness was still increasing upon termination of the test. The cumulative volume versus square root of time plot would nomally flatten out as less and less filtrate is accumulated. 
FIGURE 55

FIITRATION TEST UNIT

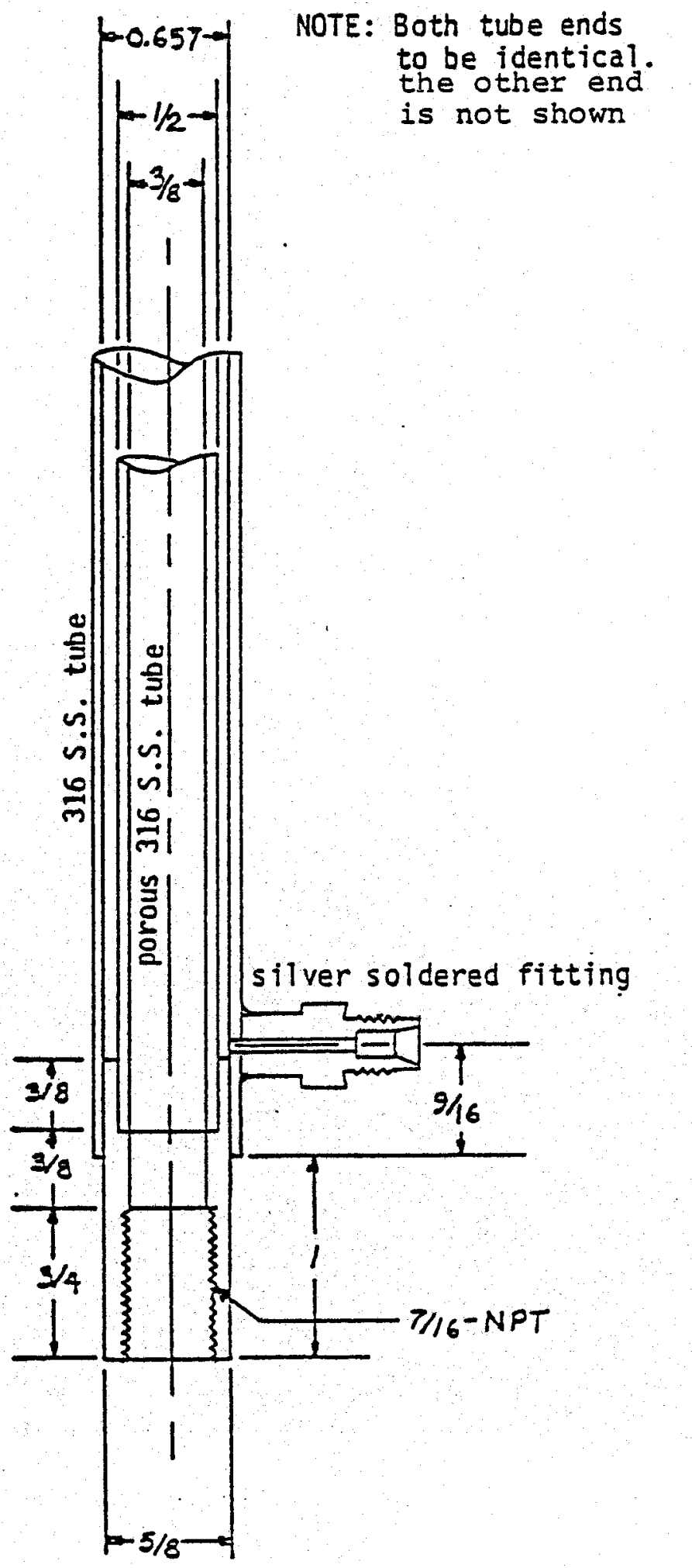


FIGURE 56

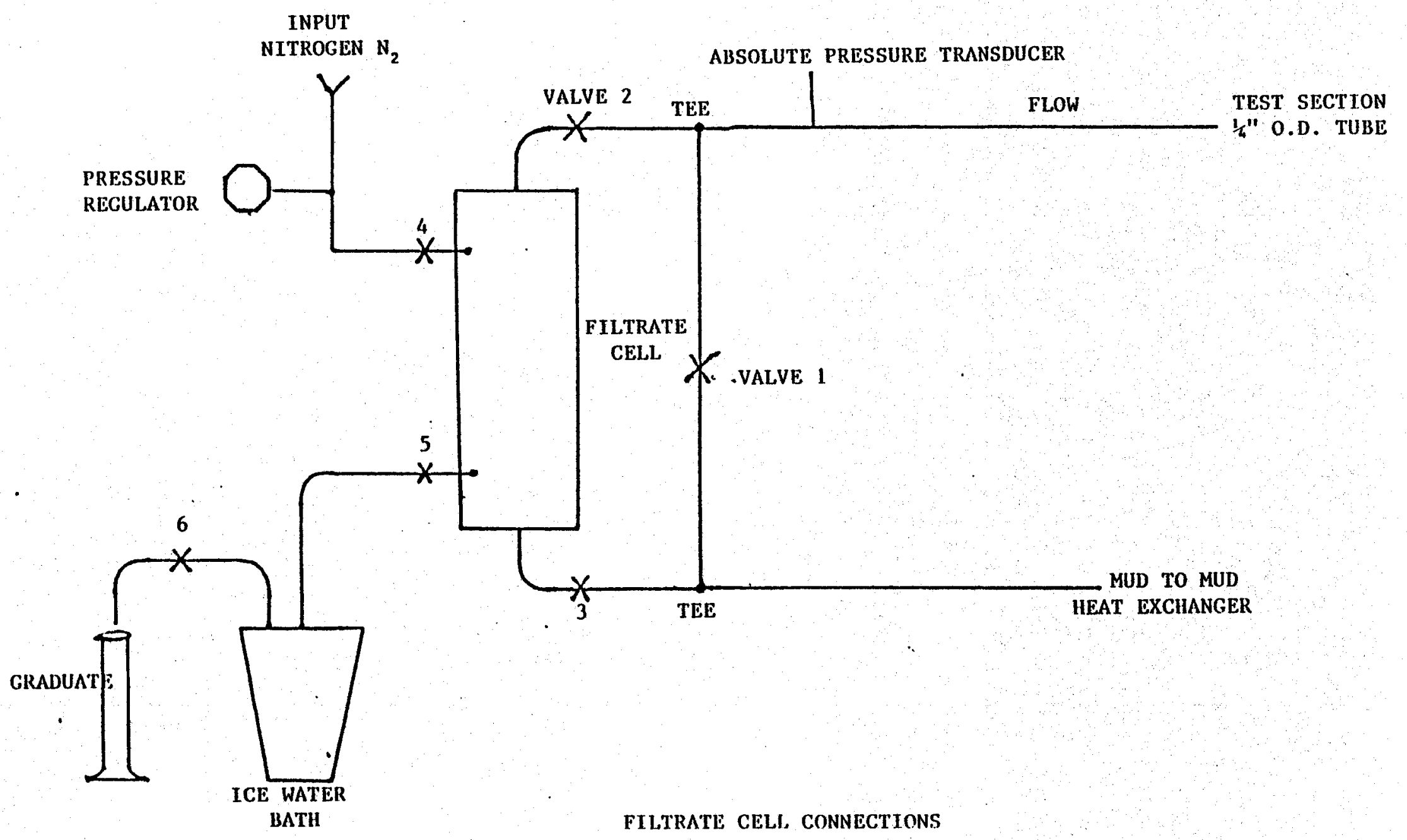


TABLE 27

FILTRATE TEST RESULTS OF CHROME LIGNOSULFONATES

\begin{tabular}{|cccc|}
\hline $\begin{array}{c}t \\
\text { time } \\
\text { (minutes) }\end{array}$ & $\sqrt{t}$ & $\begin{array}{c}\text { Filtrate } \\
\text { Volume } \\
\text { cc }\end{array}$ & $\begin{array}{c}\text { Cumulative } \\
\text { Filtrate } \\
\text { cc }\end{array}$ \\
\hline 5 & 2.24 & 21.0 & 21.0 \\
10 & 3.16 & 27.0 & 48.0 \\
15 & 3.87 & 11.2 & 59.2 \\
20 & 4.47 & 7.6 & 66.8 \\
25 & 5.0 & 5.2 & 72.0 \\
30 & 5.5 & 4.4 & 76.4 \\
& & & \\
& & & \\
\hline
\end{tabular}

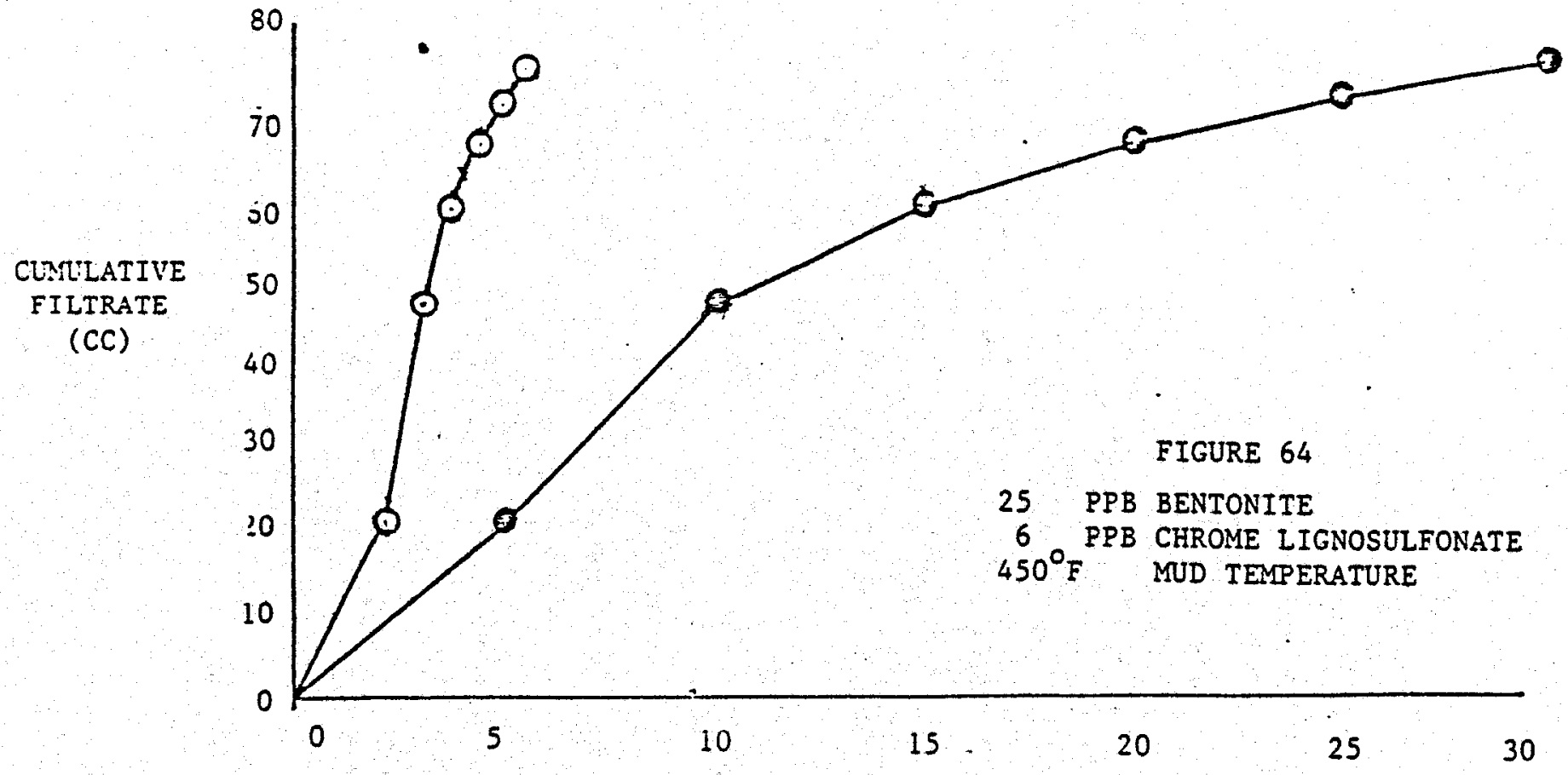

TIME (MINUTES)

- tine plot

O SQUARE ROOT TIME PLOT 
2. CORROSION TESTS

Corrosion tests made with the flow loop are of the weight loss type. The corrosion cell is illustrated as Figure 55 of Appendix $A$. The coupons used are a mild steel in a rectangular flat shape approximately $\left(0.4^{\prime \prime} \times 1.95^{\prime \prime} \times 0.043^{\prime \prime}\right)$. The coupons are weighed before and after exposure to the mud in the flow loop.

The corrosion rate can be calculated by the following equation Corrosion rate $\left(\frac{\text { in }^{3}}{\text { in }^{2}} \frac{1}{y r}\right)=\frac{\text { gran. wt. loss }}{\mathrm{A}\left(\mathrm{in}^{2}\right) \cdot \mathrm{T}(\mathrm{hrs}) \cdot(.005) \cdot(2.9)}$

C.R. (mdd) $=\left(\frac{i n^{3}}{1 n^{2}} \frac{1}{y r}\right) \cdot(1901)$

C.R. (mils per year) $=($ mdd $) \cdot(0.1826)$

Tests were run using two different types of experimental drilling fluids to establish accuracy of the test technique.

The same material coupons were used in both tests. 
TABLE 28

CORROSION TEST 非

\begin{tabular}{|ccccc|}
\hline SAMPLE \# & AREA $\left(\mathrm{in}^{2}\right)$ & WT. LOSS (gram) & C.R. (mPY) & TEMP. ${ }^{\circ} \mathrm{F}$ \\
\hline 1 & 2.025 & 0.013 & 2.95 & $100^{\circ} \mathrm{F}$ \\
2 & 2.076 & 0.009 & 2.06 & $100^{\circ} \mathrm{F}$ \\
3 & 2.072 & 0.022 & 4.95 & $300^{\circ} \mathrm{F}$ \\
4 & 1.99 & 0.023 & 5.38 & $300^{\circ} \mathrm{F}$ \\
\hline
\end{tabular}

CORROSION TEST \#\#

\begin{tabular}{|ccccc|}
\hline SAMPLE \# & AREA $\left(\right.$ In $\left.^{2}\right)$ & WT. LOSS (gram) & C.R. (mpy) & TEMP. ${ }^{\circ}$ \\
\hline 5 & 2.08 & 0.01 & 5.12 & $300^{\circ} \mathrm{F}$ \\
6 & 1.89 & 0.01 & 5.57 & $300^{\circ} \mathrm{F}$ \\
\hline
\end{tabular}


FIGURE 57

EXPERIMENTAI DRILLING FLUID TESTS
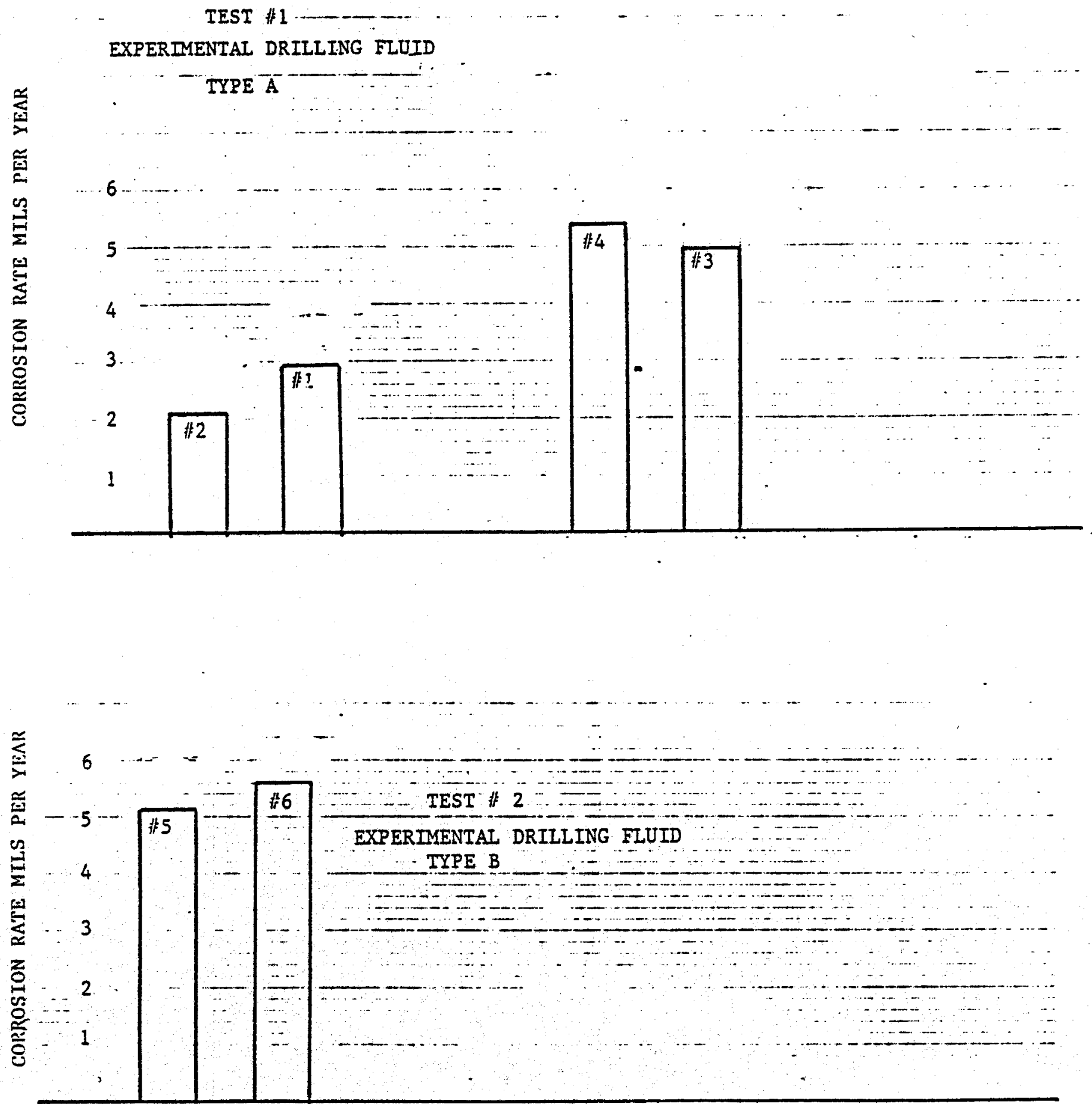

$300^{\circ} \mathrm{F}$ HIGH TEMPERATURE 
As can be seen from the bargraphs Figure 65 a substantial difference in corrosion is noted between high and low temperature. Also the Type B drilling fluid has a slightly greater corrosion effect at elevated temperatures that the Type A. The average scatter in measurement is approximately $0.3 \mathrm{mills}$ per year for this data. 
APPENDIX E 


\section{REFERENCES}

1. Adams, J. A. and Rogers, D. F.: "Computer Aided Heat Transfer Analysis", McGraw-Eill, p. 149, 1973

2. Annis, M. R.: "High Temperature Flow Properties of Water Base Drilling Fluids", J. Pet. Tech. P. 1074, August 1967

3. Anonymous, : "Study of Wyoming Bentonite", Univ. Wyoming, Natural Resources Research Institute Bull., 1946

4. "ASHRAE Bandbook of Fundamentals", Amer. Soc. Heating, Refrigerating, Air-conditioning Engineers, p. 254, 1967

5. Birch, F.: "Handbook of Physical Constants", Geol. Soc. Amer., Special paper no. 36,1942

6. Chapman, A. J.: "Heat Transfer", MacMillan, p. 530, 1967

7. Chenevert, M. E.: Personal Communication, 1977

8. Cheng, D. et al: "The Flow of Thixotropic Bentonite Suspensions Througi Pipes and Pipe Fittings", Irans. Instn. Chem. Engrs., V. 43 I 176, 1965

9. De Lautrec, J.: "Behavior of Mud Under Borehole Conditions". SPE Paper, nо. 4851,1974

10. Ede. A. J.: "An Introduction to Heat Transfer", Pergamon Press, p. 179, 1967

11. Forsythe, W. E.: "Smithsonian Physical Tables". Smithsonian Inst., p. $136,160,1954$

12. Gambill, W. R. et al: "Heat Transfer, Burnout, and Pressure Drop for Water in Swirl Flow Through Tubes with Internal Twisted Tapes" Chem. Eng. Progress Symp. Series, v. 57, no. 32, p. 127,1961

13. Gebhart, B.: "Eeat Transfer", McGraw-Hill, p. 389, 1971

14. Geiringer, P. I.: "Handbook of Heat Transfer Media", Reinhold, 1962

15. Govier, G, W. and Aziz, K.: "The Flows of Complex Mixtures in Pipes", Van Nostrand Reinhold, p. 186, 1972 
16. Gray, J. W.: "A Dynante Filtration Study of Drilling Fluids", M. S. Thesis, University of Oklahoca, p. 31, 1965

17. Hiller, K. E.: "Rheological Measurements on Clay Suspensions and Drilling Fluids at High Temperatures and Pressures", SPE Paper, no. 489, 1963

18. Holmes, E.: "Handbook of Industrial Plpework Engineering", John Wiley, p. 39,1973

19. Japikse, D.: "Advances in Thermosyphon Technology", Adv. in Heat Transfer, v. 9, p. $1-112,1973$

20. Johnson, W. B. and Nagle, W. M.: "Circulating HTS as a Pilot Plant Heat Transfer Medium", Industrial and Eng. Chem., v. 39, no. 8, 1947

21. Kays, W. M. and London, A. L.: "Compact Heat Exchangers", McGraw-Hill P. 50,1964

22. Kern, D. Q.: "Process Heat Transfer", McGraw-Hil1, 1964

23. Kirst, W. E., Nagle, W. M. and Castner, J. B., Trans A.I.C.H.E., 36, 371 (1940).

24. Klepper, O. H.: "Heat Transfer Performance of Short Twisted Tapes", Amer. Inst. Chem. Eng. Symp. Ser., v. 69, p. 87, 1973

25. Kreith, F.: "Principles of Heat Transfer", International Textbook, p. 597,1965

26. Kreith, F. and Sumerfield, M.: "Heat Transfer to Water at High Heat Flux Densities with and without Surface Boiling", ASNE Trans. v. 71, p. 805,1949

27. Krueger, R. E., "A Study of Temperature Effects Upon the Flow of a Non-Newtonian Fluid", M.S. Thesis, University of Oklahoma, 1969.

28. R. C. Lord, P. E. Minton, and R. P. Slusser, "Design of Heat Exchangers" Chemical Engineering, Jan. 26, 1970.

29. Martin, B. W.: "Free Convection in an Open Thermosyphon", Proc, Roy. Soc. London. A230, pp. 502-531, 1955

30. McAdams, W. H.: "Heat Iransmission", McGraw-HiIl, D. 243, 1954

31. Metzner, A. B. and Gluck, D. G.: "Heat Transfer to Non-Newtonian Fluids Under Laminar Flow Conditions", Chem. Eng. Sci., v. 12, p. 185, 1960

32. Orr, C. and Dalla Valle, J. M.: "Heat Transfer Properties of LiquidSolid Suspensions", Chem. Eng. Prog. Symp. Ser., v. 9, no. 50, p. 29,1954 
33. Ostrach, S.: "Natural Convection in Enclosures", Adv. in Heat Transfer, v. 8 , p. $161-227,1972$

34. Outzans, E. D.: "Mechanies of Static and Dymamic Filtration in the Bore Hole", SPE Paper no. 491, lst Conf. Drtlling Rock Mech., University of Texas, 1963

35. Rogers, W. F.: "Composition and Propertles of O1l Well Drilling Flu1ds", Gulf Publishing Co., P. 395, 1963

36. - Schack, I. A.: "Industrial Heat Transfer", p. 176, 1965

37. Seigel, I. G.: "The Effect of Turbulence Promotors on heat Transfer Coefficlents for Water Flowing in Borizontal Tubes", Heating, Piping and Air Conditioning, v. 18, P. 111, June, 1946

38. Sherman, P.: "Industrial Rheology", Academic Press, p. 46, 1970

39. Skelland, A. E. P.: "Non-Newtonian Flow and Heat Iransfer:, John Wiley, p. 386,1967

40. Taborek, J., et al: "Fouling: The Major tnresolved Problems in Heat Irussfer", Chem. Eng. Progress, v. 68, no. 2, P. 59, Feb. 1972

41. Vargaflik, N. B., Neimark, B. E., and Oleshahuk, O. N., Eulletin of IZTEST., V.I.I. 21, No. 9, 1-7 (1952).

42. Toznick, E. P. and Uhl, V. W.: "Molten Salt for Heat Transfer", Chem. Eng., v. 70, P. 129, May 1963

43. Feintritt, D.J. and Hughes, R. G.: "Factors Involved in High-Temperature Drilling Fluids", 2nd. Conf. Drilling Rock Mech., Oniv. Texas, SPE Paper no. 1043,1963

44. Wyant, R.: "A Unique System for Preparation and Evaluation of Eigi Temperature Drilling Fluids", SPE Paper, no. 5515, 1975

45. Hyant, R.: Personal Comunication, 1977

46. Fuan, S. W.: "Foundations of Fluid Mechanics", Prentice-Pall P. 273, 1967

47. Zukeuskas, A.: "Heat Transfer from Tubes in Cross Flow", Adv. in Heat Iransfer, v. 8, P. 131, 1972 
APPENDIX F

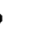

- 


\section{BIBLIOGRAPHY}

1. Abercromb1, W.F., Jr., 1974, Method for Controlling the Viscosity of Dispersed Clay Slurries: U.S. Patent 3,961,979, J. M. Huber Corp.

2. Achterberg, A., 1973, A New Salt-Based Mud System For the North Sea: 2nd Ann. SPE of AIME Europe Meeting, Preprint No: SPE-4319, p. $1-8$.

3. Alexander, H., 1973, How Coatings and Care Extend Drill P1pe Life: Drilling, (Apr. 20, 1973), V. 34, No. 7, p. 52-53, 55.

4. Arnold, 0. M., and Vancini, C. A., 1973, Gas Cleaning Apparatus: U.S. Patent 3,812,658, Peabody Engr. Corp.

5. Baillie, R. A., 1971, Method For Upgrading Bitumen Froth (I): Canadian Patent 952,837, Great Canadian 011 Sands, Ltd.

6. - - 1971, Method For Upgrading Bitumen Froth (II): Canadian Patent 952,838, Great Canadian 011 Sands, Ltd.

7. --1971, Method For Upgrading Bitumen Froth (III): Canadian Patent 952,839, Great Canadian Oil Sands, Ltd.

8. - -1971, Method For Upgrading Bitumen Froth (IV): Canadian Patent 952,840, Great Canadian Oil Sands, Ltd.

9. Batllie, R. A., and Humeniuk, E., 1971, Method of Processing Bituminous Froth Recovered From Tar Sands (I): Canadian Patent 952,841 , and 952,842 , Great Canadian 0il Sands, Ltd.

10. - -1971, Method of Processing Bituminous Froth Recovered From Tar Sands (II): Canadian Patent 952,843, Great Canadian 011 Sands, Ltd.

11. - - 1971, Method of Processing Bituminous Froth Recovered From Tar Sands (III): Canadian Patent 952,844, Great Canadian 011 Sands, Ltd.

12. Baker, C., and Withers, A., 1976, Chemical Treatment Programmes for Injection Water -- An Instrumentation Package for North Sea

Flelds: Pet. Times, (Oct. 1, 1976), V. 80, No. 2036, p. 23, 25, 27.

13. Ballard, D., 1977, Operator Talk... Glycol Dehydration: Hydrocarbon Process, (Apr. 1977), V. 56, No. 4, p. 111-118. 
14. Barthel, H.R.F., and Miller, G. L., 1974, Drilling Mud-Cement Compositions for Well Cementing Operations: U.S. Patent 3,877,009, 011 Base Inc., O1l Base Germany GMBH \& Co.

15. Baughmann, K.E., 1975, P1pe Insulating System and Apparatus Therefor: U.S. Patent $3,876,355$, B \& B Insulation Co.

16. Baumann, R., and Methven, N.E., 1973, Here's How 011 Muds Perform At High Temperatures: Pet. Petrochem Int., (Sept. 1973), V. 13, No. 9, p. $54-56,61$.

17. Behan, S.P., and Vendrinsky, D.A., 1973, Method of Treating Effluents from Hot Water Extraction of Bitumen from Tar Sands: Canadian Patent 1,000,632, Great Canadian 011 Sands, Ltd.

18. Be11, L.N., Knowles, C.R., and Miles, L.H., 1975, Well Completion in Permafrost: U.S. Patent 3,971,441, Atlantic Richfield Co.

19. Bennett, R.B., Bland, R.G., and McMordie, W.C. Jr., 1974, The Effect of Temperature and Pressure on the Viscosity of 011 Base Muds: 49 th Ann. SPE of AIME Fal1 Mtg., Preprint No. SPE-4974, p. 1-12.

20. Bernard, G. G., 1971, Method of Placing A Well On Production: U.S. Patent $3,750,753$, Union O11 Co. of California.

21. Bodine, O.K., and Sauber, C.A., 1974, Drilling Mud Having Reduced Corrosiveness: U.S. Patent 4,000,076, Phillips Pet. Co.

22. Borchert, A.E., Burt, G.R., Condo, A.C., and Kn1ght, G.R., 1971, Structure For Protecting and Insulating Frozen Substrates and Method for Producing Such Structures: U.S. App1. $\nabla 418,490$, Dec. 6, 1971.

23. Brooks, W. R., and Stonitsch, L.J., 1974, Method and Apparatus for Forming Jacketed Insulated Pipe: Canadian Patent 990,587, (Purchased by U.S. Jan. 7, 1974), Insta Foam Products Inc.

24. Bryant, W.J., Goldburn, J.R., and Hrudey, S.E., 1974, Water Pollution Aspects from Waste Driling Mud Disposal In Canada's Arctic: 6th Ann. SPE of AIME Offshore Tech. Conf., Preprint No. OTC-2044, V. 2, p. $95-106$.

25. Burland, P.D., Stephenson, J.L., and Stobart, E.H., 1972 Composition and Process for the Reduction of Thermal Degradation of Aqueous Drilling Fluids: U.S. Patent $3,764,530$, Milchem Inc:

26. Buce, W. F., 1972, Method for Restricting Fluld Pathways Through a Subterranean Formation Zone: U.S. Patent 3,771,599, Phillips Pet. Co. 
27. Cadman, T.W., and Dellinger, R. W., 1974, Techniques For Removing Metals From Process Wastewater: Chemical Engr., (Apr. 15, 1974), V. 81 , No. 8 , p. 79-85.

28. Calvert, T. J., 1974, High Frequency Method and Apparatus for Electrical Investigation of Subsurface Earth Formations Surrounding a Borehole Containing an Electrically: U.S. Patent $3,973,181$, Schlumberger Technol. Corp.

29. Carico, R.D., 1973, Cementing in 011 Muds: Baroid.News Bu11., V. 24, No. 2 , p. 9-15.

30. Carlton, L. A., and Thompson, G. D., 1971, Controlling Subsurface Pressures While Drilling With Oil Base Muds: U. S. Patent 3,750,766, Esso Prod. Res. Co.

31. Carney, L. L., 1974, Cement Spacer Flu1d: SPE of AIME Permian Basin $0 i 1$ \& Gas Recovery Conf., Preprint No. SPE-4784.

32. Carrol1, R. D., and Muller, D. C., 1973, Techniques For The Physical Properties From Geophysical Logs Southern Yucca Flat, Nevada Test Site. (Nov. 1973), Geol. Survey, Rep. No. USGS-474-175, p. 1-113.

33. Carter, L. G., and Sutoon, D. L., 1973, Method of Plugging A Well: U. S. Patent 3,844,351, Halliburton Co.

34. Chenevert, M. E., 1973, Field Performance of Oilfield Drilling Fluids: AICHE Symposuin, V. 69, No. 135, p. 200-202.

35. Clark, R. K., and Fontenot, J. E., 1974, Fleld Measurements of the Effects of Drillstring Velocity, Pump Speed, and Lost Circulation Material On Downhole Pressures: 49 th Ann. SPE of AIME Mtg., Preprint No. SPE-4970, p. 1-24.

36. Collins, A. G., 1976, Chemical Applications in 011-And-Gas-WellDrilling and Completion Operations: U. S. Environmental Protection Agency, Environmental Aspects of Chemical Use In Well Drilling Operations Conference Proceedings, p. 231-260.

37. Cook, M. A., and Eagmann, M. J., 1974, Explosive Compositions Containing Metallic Fuel Particles and Method of Preparation Thereof : U. S. Patent 3,926, 698, Ireco Chemicals.

38. Cooper, R. E., 1973, Methods For Forcing A Liquid Into A Low Pressure Formation: U. S. Patent $3,863,717$, CIE Dowell Schlumburger.

39. Congram, G. E., 1975, Processors Counterattack Amine-Unit Corrosion: 011 \& Gas Jour., (Feb. 10, 1975), V. 73, No. 6, p. 55, 56. 
40. Cox, J. W., and Raymer, L. L., 1976, The Effect of PotassiumSalt Muds on Gamma Ray, and Spontaneous Potential Measurements: 17 th Ann. SPWLA Logging Symp. Trans., Paper No. 11, p. 1-20.

41. Cox, T. E., and McClothlin, R. E., 1973, Drilling Fluids, Additives Therefor, and Stabilization Methods Therefor: U. S. Patent $3,878,111$, Dresser Indus. Inc.

42. Cranfleld, J., 1973, Cuttings Clean-Up Meets Offshore Pollution Specifications: Petroleum Petrochem Int., (Mar. 1973), V. 13, No. 3, p: 54-56.

43. Creaser, L. F., 1971, Flexible Insulants For Containers and Conduits: U. S. Patent $3,810,839$, Shell 0 il Co.

44. Curtin, D. J., 1974, Method For Tar Sands Bitumen Froth Treatment: U. S. Patent 3,813, 030, Atlantic Richfield Co.

45. Darley, H. C. H., Harrison, G., and Hartfiel, A. H., 1974, Ac1dSoluble Drilling, Completion and Workover Fluids: 21st Ann. Pet. Short Course Assoc., p. 35-39.

46. Darley, H. C. H., and Hartfiel, A., 1974, Tests Show That Polymer Fluids Cut Drill-Plpe Pressure Losses: 011 \& Gas Jour., (June $17,1974)$, V. 72 , No. 24, p. $70-73$.

47. Davies, J. T., and Thomas, T. B., 1974, on The Sudden Stretching of Liquid Lamellae: Jour. Colloid Interface Sclence, (Sept. 1974), v. 48 , No. 3, p. $427-436$.

48. Davitt, H. J., 1972, Recovery of Bitumen From Sludge Resulting From Hot Water Extraction of Tar Sands: Canadian Patent 975,696, $975,697,975,698975,699$, and 975,700, Great Canadian Oil Sands Ltd.

49. - - 1972, Hot Water Extraction and Hydrocyclone Treatment of Tar Sands: Canadian Patent 970,308, Great Canadian 011 Sands, Ltd.

50. Dickson, D., 1976, Drilling the Contlnental Shelf: Barold News Bu11. (Winter 1976), V. 27, No. 4, p. 20-24.

51. Donham, J. D., 1972, Polymer Non-Dispersed Drilling Fluids: U. S. Patent $3,880,764$, Amoco Prod. Co.

52. Donham, J. E, , 1976, Chemical Control of Drillpipe Corrosion: International NACE Corrosion 76 Conference, Preprint No. 1, p. 110.

53. Douglass, J. H., Walker, C. 0., and Walker, T. 0., 1973, Method of Drilling Wells Employing Water Base Drilling Fluids: U. S. Patent 3,953,337, Texaco Inc. 
54. Drilling, 1973, How To Administer A 3000-BBL Transfusion: Drilling, (June 1973), V. 34, No. 9, p. 56.

55. Du Charme, I., and Jackson, J. G., 1974, We11 Drilling Method and Apparatus: U. S. Patent 3,889,764.

56. Ewing, R. C., 1975, Hot-01I Pipeline System Nearly Completed: Oil \& Gas Jour., (Sept. 8, 1975), V. 73, No. 36, p. 66-68, 70, 72.

57. Finley, B. M., and Sturdivan, P. G., 1971, Method And Apparatus For Cleaning Solids Coated With Oil: U. S. Patent 3,716,480, Demco Inc.

58. Fontenot, J. E., and Simpson, J. P., 1973, A Microbit Investigation of The Potential For Improving The Driling Rate of 0il-Base Muds In Low Permeability Rocks: 48th Ann. SPE of AIME Fall Meeting, Preprint No. SPE-4519, p. 1-12.

59. Franco, A., 1977, Premex Optimfzes Reforma Operations: 011 \& Gas Jour., (Mar. 28, 1977), v. 75, No. 13, p. 135-140.

60. Franco, A., and White, R. W., 1975, Pheological Agent for NonAqueous Fluid Systems: U. S. Patent $3,977,894$, N.L. Industries Ine.

61. Fraser, W. M., 1973, Apparatus For Overcoming Lost Circulation In Oil Wells: U. S. Patent 3,726,340.

62. Grantham, K., and Shoults, L., 1976, How To Analyze O1l Mud: Contamination Solids: Drilling, (July 1976), V. 37, No. 10, p. 51-53.

63. Gray, G. R., 1973, New Methods Designed To Improve Drilling Rate, Hole Stability: World 0il, (May, 1973), V. 176, No. 5, p. 84-85.

64. Guffy, J. C., Paulson, M. H., and Winkler, R. A., 1974, Process Improvement in the Absorption of Acld Gas from a Feed Gas: U. S. Patent $4,002,721$, Chevron Research Co.

65. Hale, D., 1974, Insulated Underwater Line Laid For IIAPCO In Java Sea: Pipeline Gas Jour., (Mar. 1974), V. 201, No. 3, P. 41-43, 47.

66. Hanni, G., 1973, Drilling The Permafrost: Baroid News Bu11,, V. 24, No. 4, p. $11-13$.

67. Hartfiel, A. H., 1974, Clay-Free Thixotropic Wellbore Fluid: U. S. Patent $3,988,246$, Chemlcal Additives Co.

68. Holroyd, L.M., 1971, Improvements in the Laying of P1pes: Great Britian Patent 1,392,931. 
69. Jones, L. W., 1972, Waterflooding Process: U. S. Patent 3,817,331, Amoco Prod. Co.

70. Juvkam-Wold, H. C., 1974, Treatment of Welighted Drilling Mud: U. S. Patent 3,964,557, Gulf Research and Devel. Corp.

71. Kablaou1, M. S., and Kolgian, J. H., 1974, Drilling Fluid, U. S. Patent 3,957,660, Texaco Ine.

72. Kablaoui, M. S., and McCoy, D. R., 1974, Drilling Fluid: U. S. Patent $3,920,560$, Texaco Inc.

73. Kang, K. S., and McNeely, W. H., 1974, Well Drilling Fluid and Method: Great Britian Patent 1,416,013.

74. Kelly, J., Jr., 1973, Method For Controlling Lost Circulation: U. S. Patent $3,724,565$, Mobil Oil Corp.

75. Kennedy, J. L., 1974, 011 Mud "Under-Balancing" Saves Money: (Feb. 11, 1974), Oil \& Gas Jour., V. 72, No. 6, p. 49-52.

76. - -1974, Placid's Dutch Gas Field Near Produciton: Oil \& Gas Jour., (June 3, 1974), V. 72, No. 22, p. 114-116.

77. Klimentov, P. P., 1973, Hydraulic Fracturing For Underground Gasification of Coal Deposits: Report No. SLA-73-6002, p. 1-24.

78. KIjucec, N. M., Yukowski, K. J., and Lipsett, L. R., 1974, Successful Drilling of Permafrost with a Bentonite-XC Polymer- $\mathrm{KCl}$ Mud System: Jour. Canad1a Petr. Tech., (Jan.-Mar. 1974), V. 13, No. 1, p. 4953 .

79. Kowalski, T., 1974, Scrap Tire Floating Breakwaters: Floating Breakwaters Conf. Proc. p. 233-246.

80. Krause, H. R., 1974, How To Minimize Damage: Drilling, (June 1974), V. 35, No. 9, p. $54,57$.

81. Kurihara, T., and Nishimaki, K., 1972, Method and Structure for Thermally Insulating Low Temperature Liquid Storage Tanks: U. S. Patent $3,895,146$, Bitachi Ship Building and Eng. Ltd.

82. Lange, G. R., 1973, An Investigation of Core Drilling In Perennially Frozen Gravels and Rock: Cold Reglons Research \& Engr. Lab, (Dec. 1973) No. CRREL-TR-245, p. 1-33.

83. Lawson, J. B., RIchardson, E. A., and Suman, G. 0., 1972, Bonding Casing With Syntactic Epoxy Resin: U. S. Patent 3,782,466, Shel1 OII Co. 
84. Leyendecker, E. A., and Murray, S. C., 1974, Properly Prepared Oil Muds Ald Massive Salt Drilling: World Oil, (April 1975), V. 180, No. 5, p. 93-95.

85. Lokshin, E. L., Starinsky, A. A., and Volk, A. F., 1975, Method of Opening Carbon-Bearing Beds with Production Wells For Underground Gasification: U. S. Patent 4,003,441.

86. Mahley, H. S., 1975, Fight Tank Fires, Subsurface: Hydrocarbon Process, (Aug. 1975), V. 54, No. 8, p.72-75.

87. Martin, R. T., 1973, Improvements In Or Relating to the Removal of Explosive or Combustible Gas or Vapor from Tanks and Other Enclosed Spaces: Great Britian Patent 1,433,815, Watler Kidde Co., Ltd.

88. Mathews, C. W., and Vind, H. P., 1973, Materials For Leak-Proofing Navy Oil Tankers (Supplement): U. S. Naval Civil Engr. Lab (July 1973) Rep. No. NCEL-TN-1252-S, p. 1-11.

89. McClain, L., 1973, Plumbing The Depths of Liquid Level Control: Process Eng. (Dec, 1973), p. 82-87.

90. McCollum, R. W., 1974, Well Drilling and Precompletion Method: U. S. Patent 3,921,719, Otis Eng. Corp.

91. McCoy, J. N., 1973, Anlyzing Well Performance VI: 20th Ann. Southwestern Pet. Short Course Assoc. Meeting Proc., p. 193-210.

92. McGuire, W. J., 1976, Disposal of Drilling Fluids and Drilled-Up Solids in offshore Drilling Operations: U. S. Environmental Protection Agency, Environmental Aspects of Chemical Use In Well-Drilling Operations Conference Proceedings, p. 523-531.

93. McMordie, W. C., Jr., 1976, Handling and Treatment of Oil-Base Drilling Muds: U. S. Environmental Protection Agency, Environmental Aspects of Chemical Use in Well-Drilling Operations Conference Proceedings, p. 505-514.

94. Methven, N., and Schumann, C., 1973, Theoretical, Laboratory and Performance Characteristics of a Seawarer-Salt Base Mud System for the North Sea: 2nd Ann. SPE of AIME Europe Meeting, Preprint No. SPE-4318, P. $1-16$.

95. Miles, I. H, 1975, Well Completion in Permafrost: U. S. Patent $3,973,626$, Atlantlc Richfield Co.

96. Miranda, S. W., 1970, Process for Controlling Oil Slicks: U. S. Patent $3,886,067$. 
97. Mohtad1, M. F., and Rao, P. N., 1973, Effect of Temperature On Flocculation of Aqueous Dispersions: Water Reserve, (May 1973), v. 7 , No. 5 , p. 747-767.

98. Mondshine, T. C., 1973, Method of Producing and Using a Gelled 011 Base Packer Flu1d: U. S. Patent $3,831,678$, N. I. Industry Inc.

99. Morris, E. F., and Motley, H. R., 1973, Oil Base Spacer System For Use In Cementing Wells Containing Oil Base Drilling Muds: 48th Ann. SPE of AIME Meeting, Preprint No. SPE4610, p. 1-8:

100. Needham, R. B., 1973, Method of Recovering 0il Using Steam: U. S. Application B529,836, Phillips Pet. Co.

101. Nevins, M. J., and Remont, L. J., 1976, Barold Arctic Casing Pack: Barold News Bull., (Spring 1976), V. 27, No. 1, p. 10-14.

102. Noma, T., 1973, Insulated Low Temperature Containers: Great Britian Patent 1,317,737, Hitachi Shipbuilding \& Eng. Ltd.

103. O11 and Gas Journal, 1973, CGA Shows Vast Array of Spill-Fighting Hardware Ready To Go On Gulf Coast: Oil \& Gas Jour., (Oct. 15, 1973), V. 71 , No. 42 , p. 132-133.

104. --1974, Technology for Hire: 011 \& Gas Jour., (Sept. 9, 1974), v. 72 , No. 39, p. $41-54$.

105. - - 1974, Iran Drilling Is A Study In Extremes: 011 \& Gas Jour., (Sept. 23, 1974), V. 72, No. 38, p. 141-142.

106. --1974, Problems Vary In Indonesia/Malaysia: 0il \& Gas Jour., (Sept 23, 1974), V. 72, No. 38, p. 134-135.

107. - - 1974, Prices Stimulate Californla 011 Recovery: 011 \& Gas Jour., (Oct. 7, 1974), V. 72 , No. 40, P. 34-35.

108. -- 1975, Downhole Rock, Flulds To Govern Deep Drilling: 011 \& Gas Jour., (June 30,1975 ), V. 73 , No. 26, p. 60.

109. Outmans, H. D., 1974, Spot Fluid Quickly To Free Differentially Stuck P1pe: 011 \& Gas Jour., (July 15, 1974), V. 72, No. 28, p. 65-68.

110. Palmer, J. D., 1973, Inhibitors: Think Before You Add Them: Canadian Chemical Process, (Feb. 1973), v. 57, No. 2, p. 58, 60, 62.

111. PIng, W. H., 1973, Method and Apparatus For Cleaning A Producing Well: U. S. Patent $3,822,750$, Texaco Inc. 
112. Rance, G. H., 1971, Well Drilling Composition: Australian Patent 469,353 .

113. Rao, D. V., and Sankaran, K. S., 1974, Mechanistic Response of Expansive Clays: Soil Science, (Nov. 1974), V. 118, No. 5, P. 289-298.

114. Reid, C. A., 1973, Recent Applications of Brines and Treated Brines In Permian Basin Drilling: 20th Ann. Southwestern Pet. Short Course Assoc. Meeting Proc., p. 41-49.

115. Richardson, E. A., Scheuerman, R. F., and Suman, G. O., Jr., 1973, Oil Base Drilling Fluid Filtrate-Effected Sand Consolidation: U. S. Patent $3,750,768$, Shell 011 Co.

116. Rike, J., 1975, How To Choose Workover, Completion Fluids: 011 \& Gas Jour., (Sept. 29, 1975), จ. 73, No. 39, p. 83, 84, 89. 90.

117. Rose, D. E., and Davitt, H. J., 1971, Frustro-Conical Baffle for Hot Water Extraction Vessel: Canadian Patent 948,133, Great Canadian 011 Sands Ltd.

118. Russell, K. L., 1973, Control of Collfgative Properties of Dr1lling Mud, U. S. Patent 3,841,419, Citles Service O1l Co.

119. Schweiger, R. G., 1973, Drilling Fluid and Method: Oं. S. Patent $3,726,796$, Kelco Co.

120. Simpson, J. P., 1976, Drilling Fluid Princlples and Operations: U. S. Environmental Protection Agency, Environmental Aspects of Chemical Use in WE1I Drilling Operations Conference Proceedings, (Sept. 1976), p. 61-71.

121. Smith, B. 0., 1973, Continuous Coiled-Tubing Unit Aids Well Cleanout: 011 \& Gas Jour., (May 14, 1973), V. 71, No. 20, p. 72-75.

122. Smith, R. H., 1973, Tar Sands Treatment: Canadian Patent 993,392, (Purchased by U. S. July 9, 1973), Atlantic Richfield Co.

123. - 1973, Tar Sands Bitumen Froth Treatment: U. S. Patent $3,808,120$ Atlantic Richfield Co.

124. Smith, R. S., 1975, offshore Production Facilities, Conclusion, Safety Systems Demand Extensive Design Study: 011 \& Gas Jour., (Spet. 15, 1975), V. 73, No. 37, p. 194, 196, 198-200.

125. Smithey, C. M., 1973, Method of Well Drilling and Composition Therefor: U. S. Patent $3,986,964$, Texas Brine Corp.

126. Sullivan, F. F., 1970, Drilling Fluid Treatment: Canadian Patent 972,141, (Purchased by U. S. Mar. 23, 1970), Rotary Drilling Serv. Inc. . 
127. Sutton, D. L., 1973, Lightweight Cellular Cement Compositions and Methods of Casting the Same: U. S. Patent $3,979,217$, Halliburton Co.

128. Swain, F. M., 1974, Marsh Gas From the Atlantic Coastal Plain: Book Editions Tech., Paris, p. 673-687.

129. Texaco Development Corp., 1972, Low Solids Shale Controlling Drilling Fluid: Great Britian Patent 1,404,112, (Purchased by U. S. Nov. 24, 1972), Texaco Devel. Corp.

130. Tschirley, N. K., 1975, Cementing in 0il Muds: Petroleum Eng., (May 1975), V. 47, No. 5, P. 140, 142, 144.

131. Walker, T. O., 1972, Aqueous Drilling Fluld and Method of Use Thereof: 0. S. Patent $3,760,892$, Texaco Inc.

132. - - 1973, Controlling Salt Contaminated Drilling Fluids By the Addition of an Admixture of a Water Soluble Chromate and Ethylene Dicarboxylic Acid: U. S. Patent 3,879,299, Texaco Inc.

133. Walker, T. 0., and Weiss, W. J., 1974, Drilling Fluids Containing Phosphate Ester Acids: South African. Patent 742,914, (Purchased by the U. S. May 25, 1973) Texaco Devel. Corp.

134. Westwood, E. B., 1971, Drilling Composition and Process for Drilling Wells Therewith: Great Britian Patent 1,334,266, Georgia Pacific Corp.

135. World Oil, 1976, Burning Oil Muds May Solve Disposal Problems: World Oil, (Jan. 1976), V. 182, No. 1, P. 112-113.

136. Yamamoto, K., 1973, Heat Insulating Wall Low Temperature Liquefied Gas Tanks: Great Britian Patent 1,375,521, Bridgestone Lpg. Co. Ltd.

137. Yueh, M. H., and Jordan, W. A., 1973, Fluid Loss Control Agent: U. S. Patent $3,733,274$, General Mills Inc.

138. Zika, H. T., 1973, Surfactant Composition: U. S. Patent 3,746,096, Carbide Corp. 